Supporting Information for

\title{
Synthesis of Organometallic Oligonucleotides through Oximation With Metalated Benzaldehydes
}

\author{
Sajal Kumar Maity and Tuomas A. Lönnberg* \\ Department of Chemistry, University of Turku, Vatselankatu 2, 20014 Turku, Finland \\ *Email: tuanlo@utu.fi
}




\section{Contents}

Figure S1. ${ }^{1} \mathrm{H}$ NMR spectrum of compound $(5 S)-5 \quad$ S3

Figure S2. ${ }^{13}$ C NMR spectrum of compound $(5 S)-5 \quad$ S5

Figure $S 3 .{ }^{1} \mathrm{H}$ NMR spectrum of compound $(5 R)-5 \quad$ S6

Figure S4. ${ }^{13} \mathrm{C}$ NMR spectrum of compound $(5 R)-5 \quad$ S8

Figure S5. ${ }^{1} \mathrm{H}$ NMR spectrum of compound $6 \ldots$

Figure S6. ${ }^{13} \mathrm{C}$ NMR spectrum of compound $6 \quad$ S11

Figure S7.1D NOESY spectrum of compound 6 with selective excitation of H1 $\quad$ S12

Figure S8. 1D NOESY spectrum of compound 6 with selective excitation of H4 $\quad$ S12

Figure S9. ${ }^{1} \mathrm{H}-{ }^{1} \mathrm{H}$ COSY spectrum of compound $6 \quad$ S13

Figure S10. ${ }^{1} \mathrm{H}-{ }^{13} \mathrm{C}$ HSQC spectrum of compound $6 \quad$ S13

Figure S11. ${ }^{1} \mathrm{H}$ NMR spectrum of compound $7 \quad$ S14

Figure S12. ${ }^{13}$ C NMR spectrum of compound $7 \quad$ S16

Figure S13. ${ }^{1}$ H NMR spectrum of compound $8 \quad$ S16

Figure S14. ${ }^{13} \mathrm{C}$ NMR spectrum of compound $8 \quad$ S18

Figure S15. ${ }^{1} \mathrm{H}$ NMR spectrum of compound 9 $\quad$ S19

Figure S16. ${ }^{13} \mathrm{C}$ NMR spectrum of compound $9 \quad$ S21

Figure S17. ${ }^{1} \mathrm{H}$ NMR spectrum of compound 10 $\quad$ S22

Figure S18. ${ }^{13} \mathrm{C}$ NMR spectrum of compound $10 \quad$ S23

Figure S19. ${ }^{1} \mathrm{H}$ NMR spectrum of compound 1

Figure S20. ${ }^{13} \mathrm{C}$ NMR spectrum of compound $1 \quad$ S26

Figure S21. ${ }^{1} \mathrm{H}$ NMR spectrum of compound 11 $\quad$ S26

Figure S22. ${ }^{13}$ C NMR spectrum of compound 11 $\quad$ S29

Figure S23. ${ }^{1}$ H NMR spectrum of compound $2 \quad$ S29

Figure S24. ${ }^{13} \mathrm{C}$ NMR spectrum of compound 2 $\quad$ S33

Figure S25. ${ }^{31} \mathrm{P}$ NMR spectrum of compound $2 \quad$ S36

Figure S26. HPLC traces of crude and purified oligonucleotide ON1-Hg _ _ S $\quad$ S36

Figure S27. Measured and simulated mass spectra of purified oligonucleotide ON1-Hg S37.

Figure S28. HPLC traces of crude and purified oligonucleotide ON1-Pd _ $\quad$ S38

Figure S29. ${ }^{31}$ P NMR spectrum of purified oligonucleotide ON1-Pd $\quad$ S38

Figure S30. Measured and simulated mass spectra of purified oligonucleotide ON1-Pd S39 

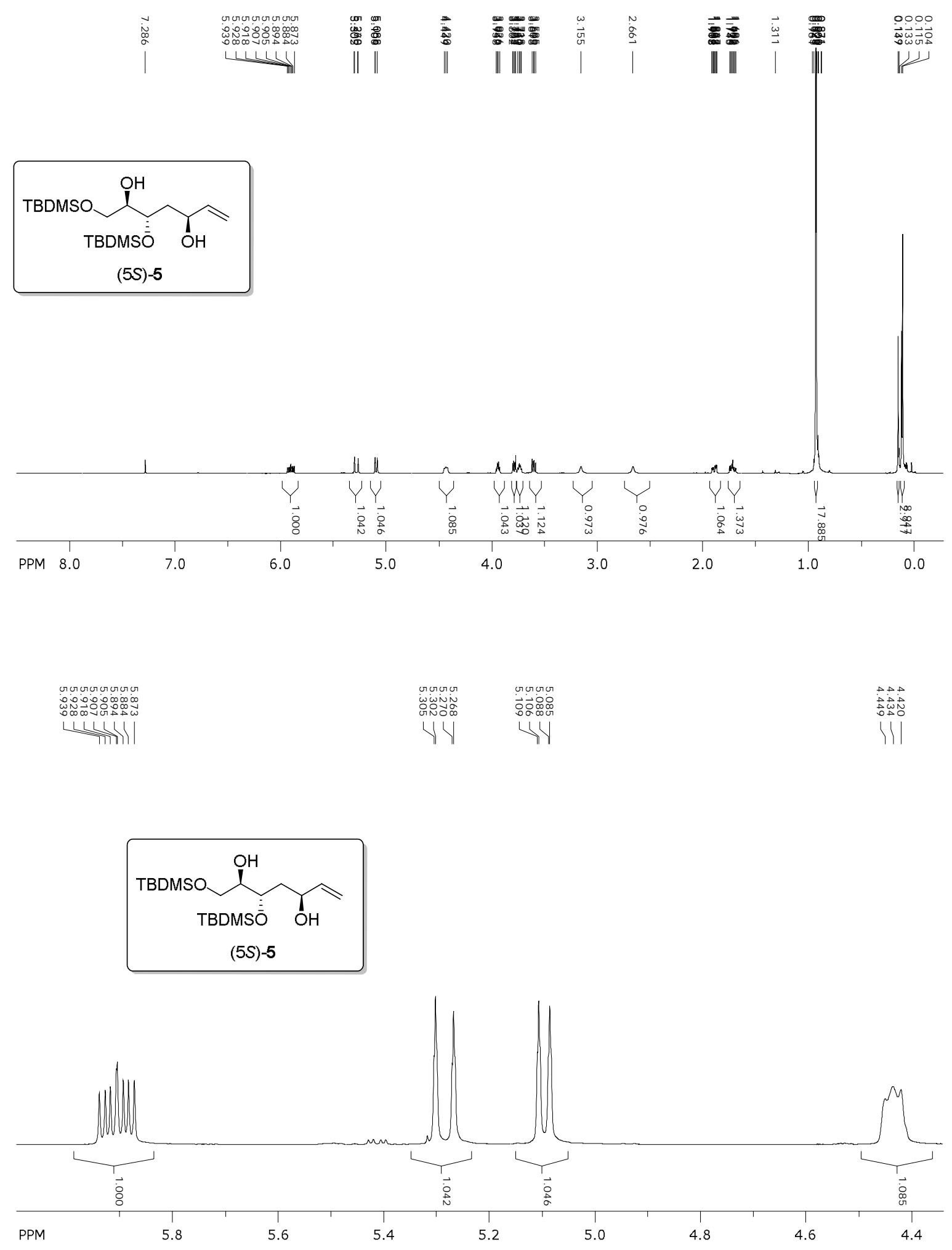

Figure S1. ${ }^{1} \mathrm{H}$ NMR spectrum of compound (5S)-5. 

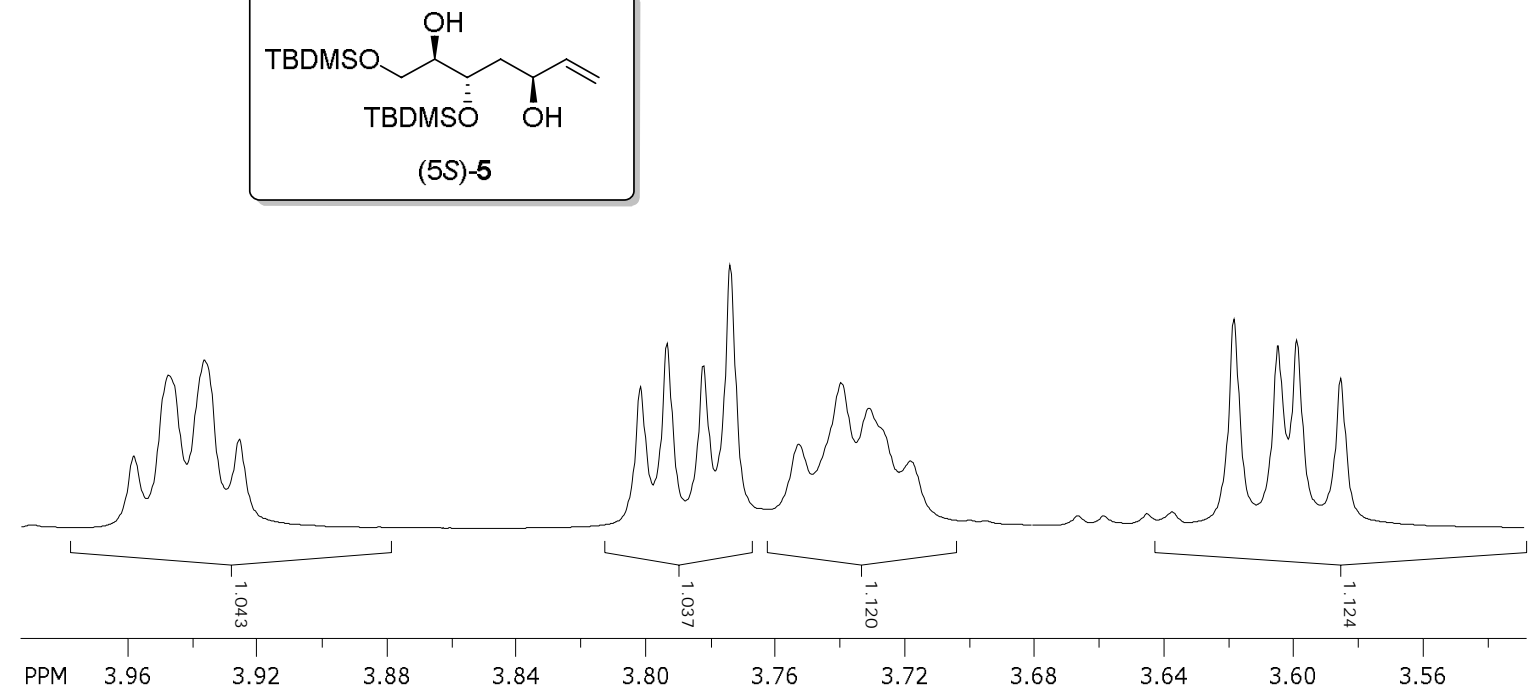

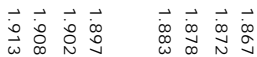
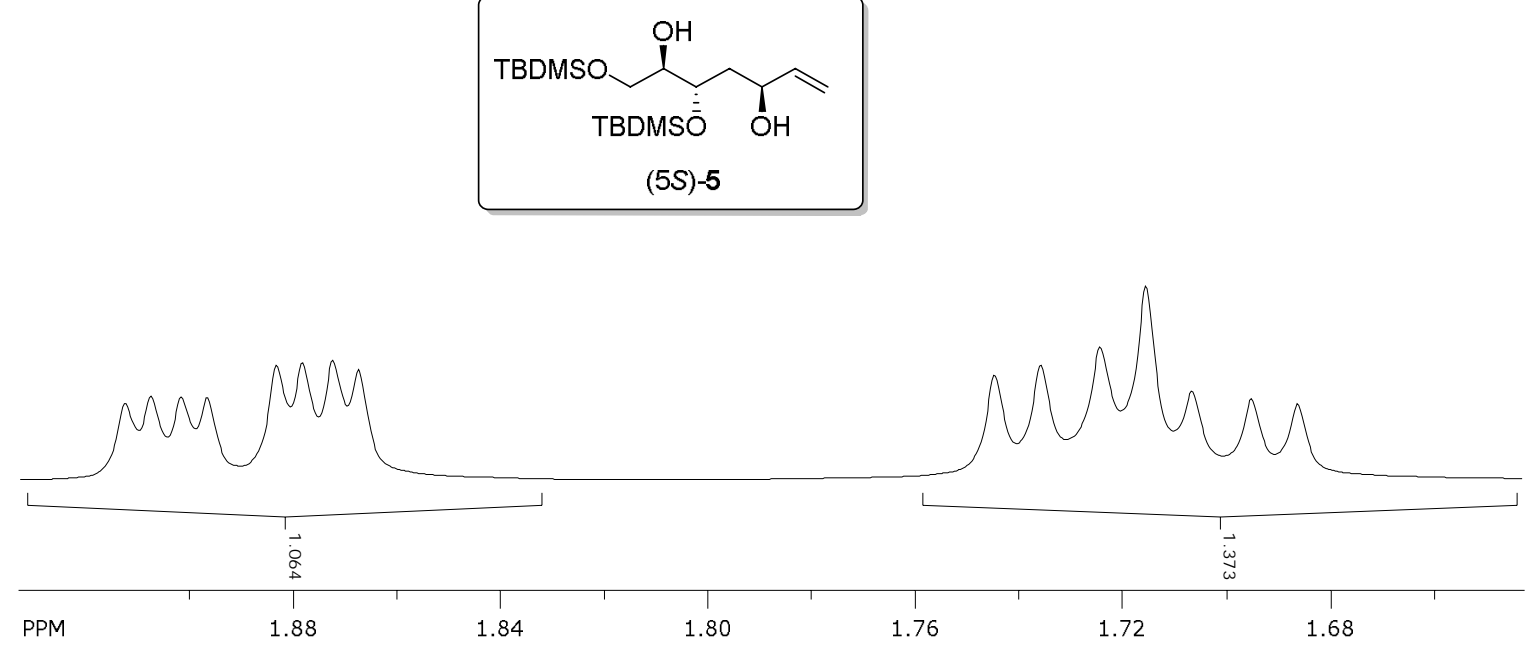

Figure S1. ${ }^{1} \mathrm{H}$ NMR spectrum of compound (5S)-5 (continued). 


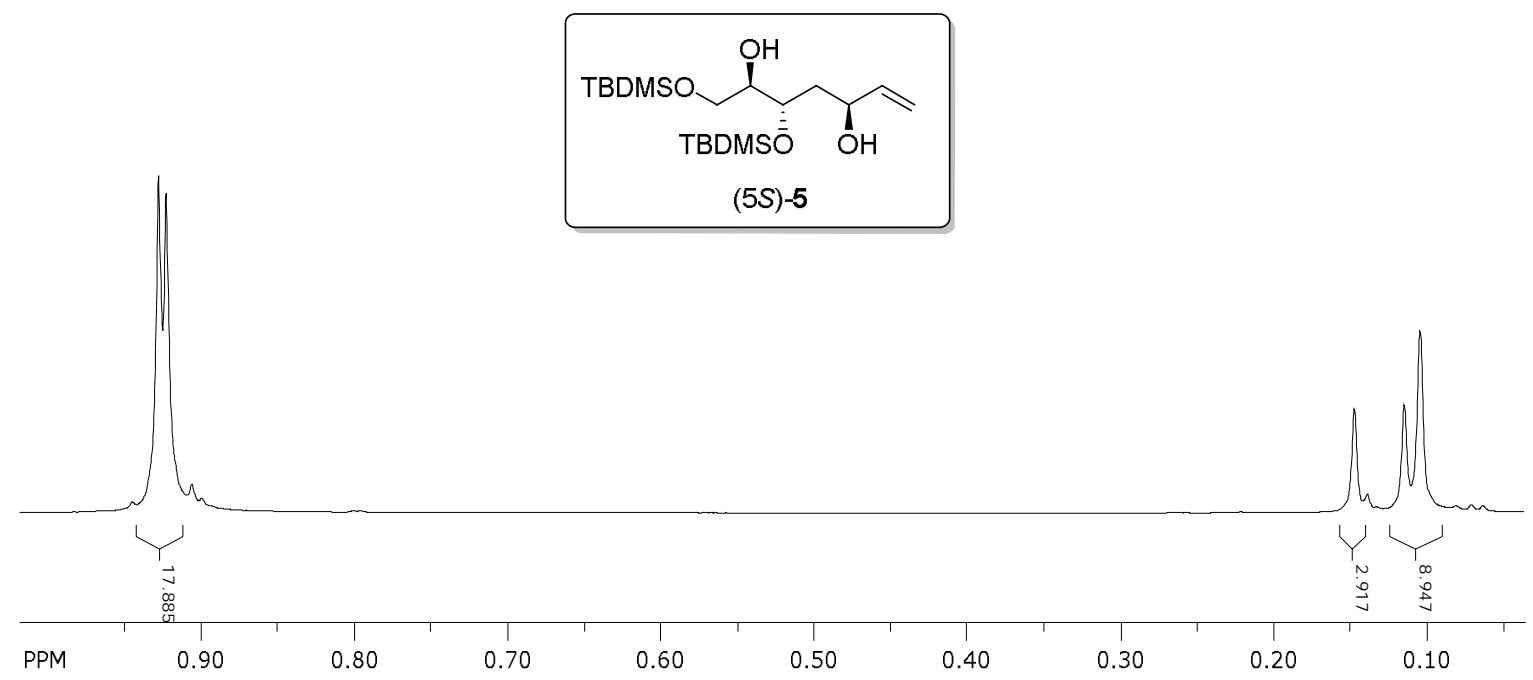

Figure S1. ${ }^{1} \mathrm{H}$ NMR spectrum of compound (5S)-5 (continued).

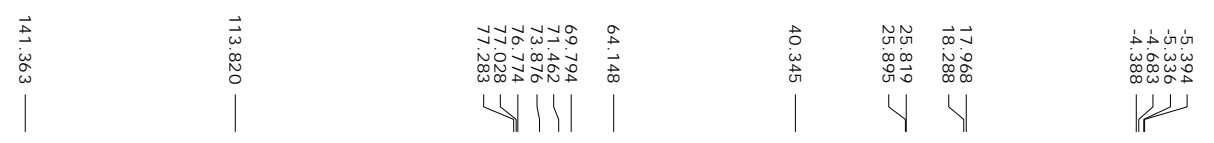

$$
\underbrace{\text { TBH }}_{\substack{\text { TBDMSŌ } \\(5 S)-5}}
$$

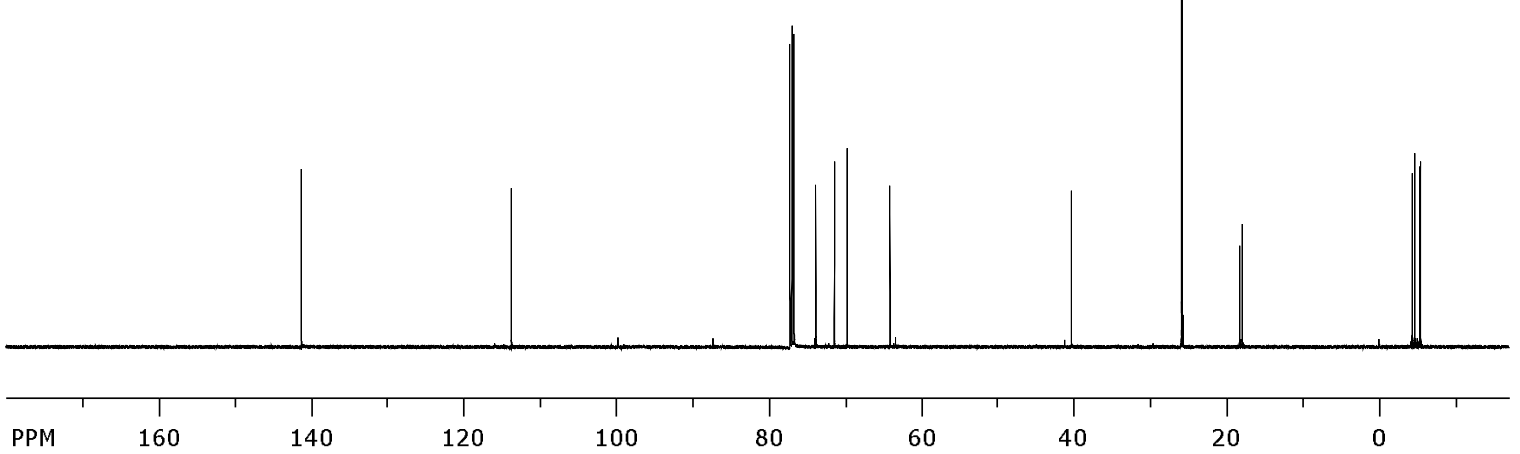

Figure S2. ${ }^{13} \mathrm{C}$ NMR spectrum of compound $(5 S)-\mathbf{5}$. 


芯
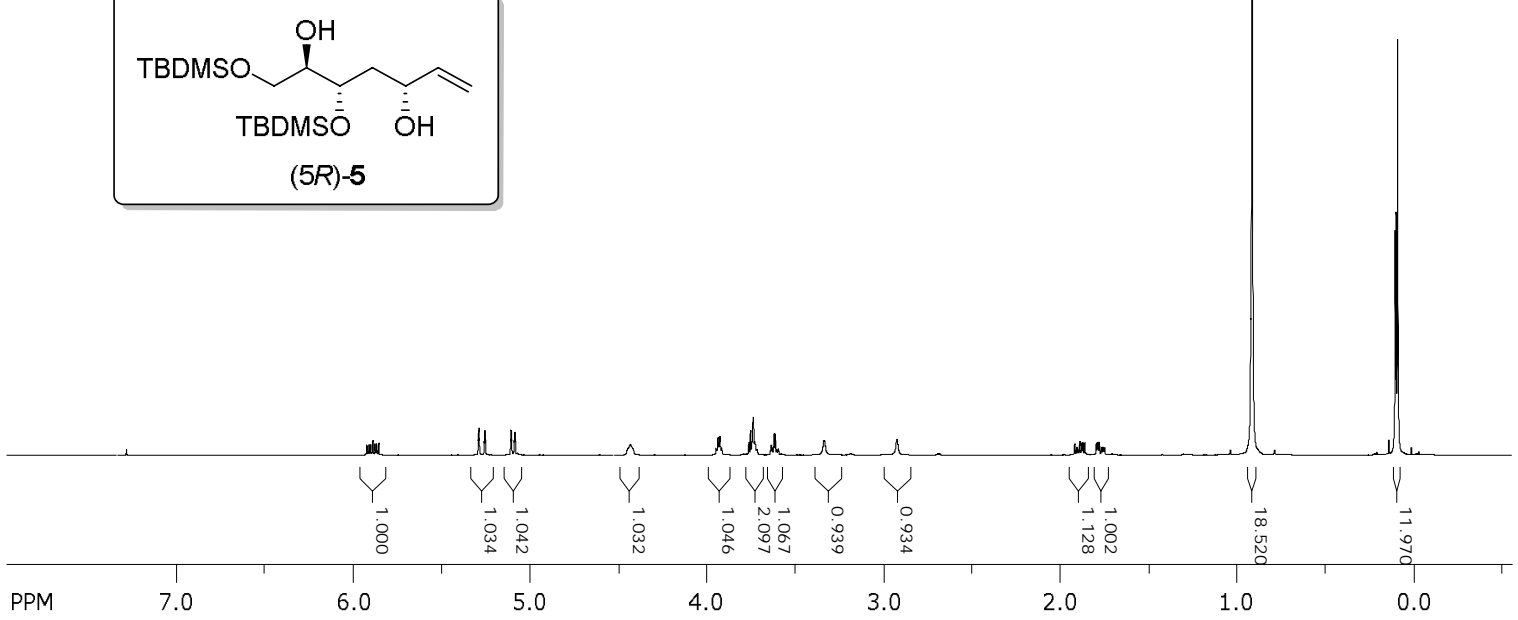

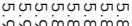

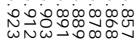

내ㄴㅔㅣㄴ
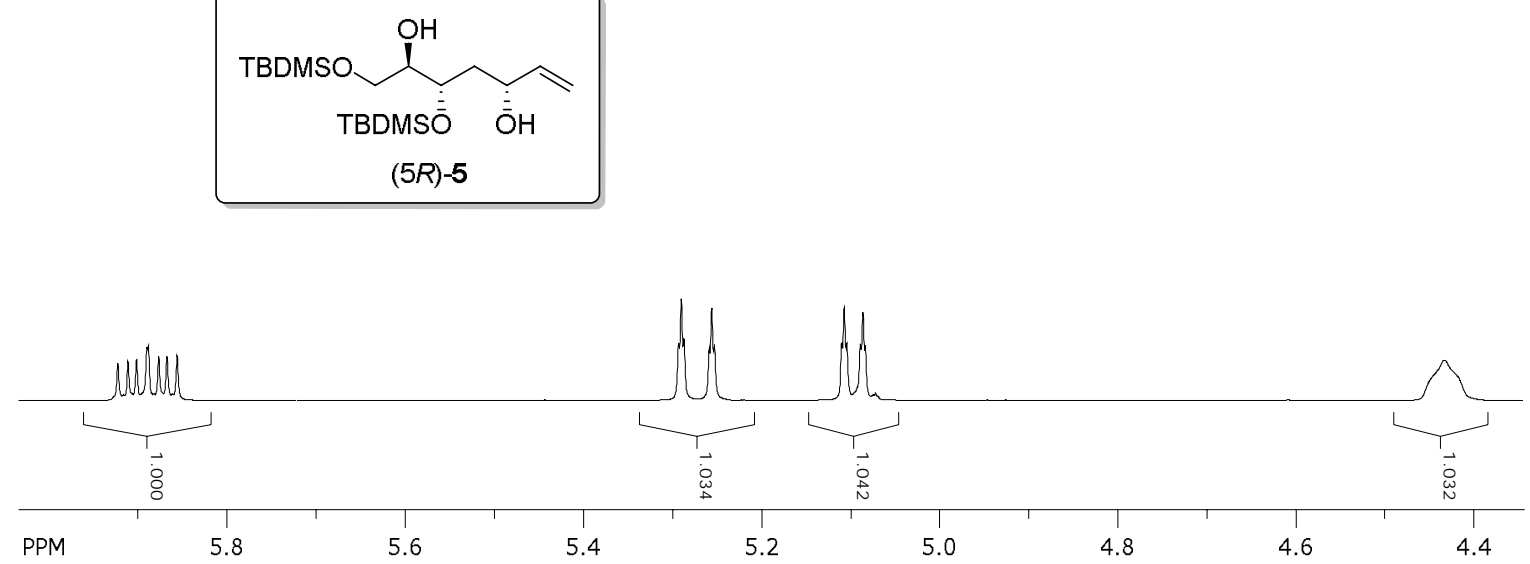

Figure S3. ${ }^{1} \mathrm{H}$ NMR spectrum of compound (5R)-5. 

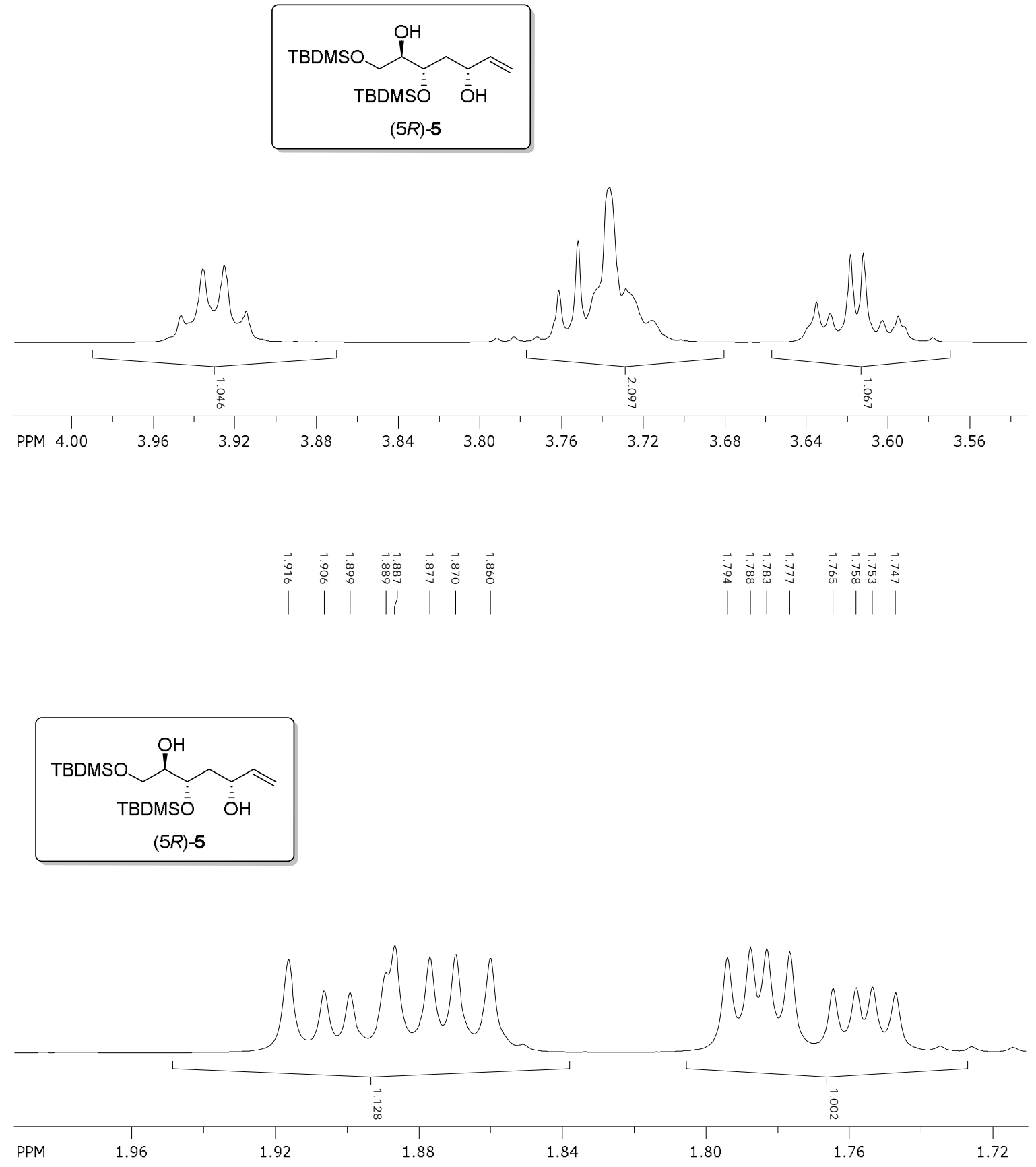

Figure S3. ${ }^{1} \mathrm{H}$ NMR spectrum of compound (5R)-5 (continued). 


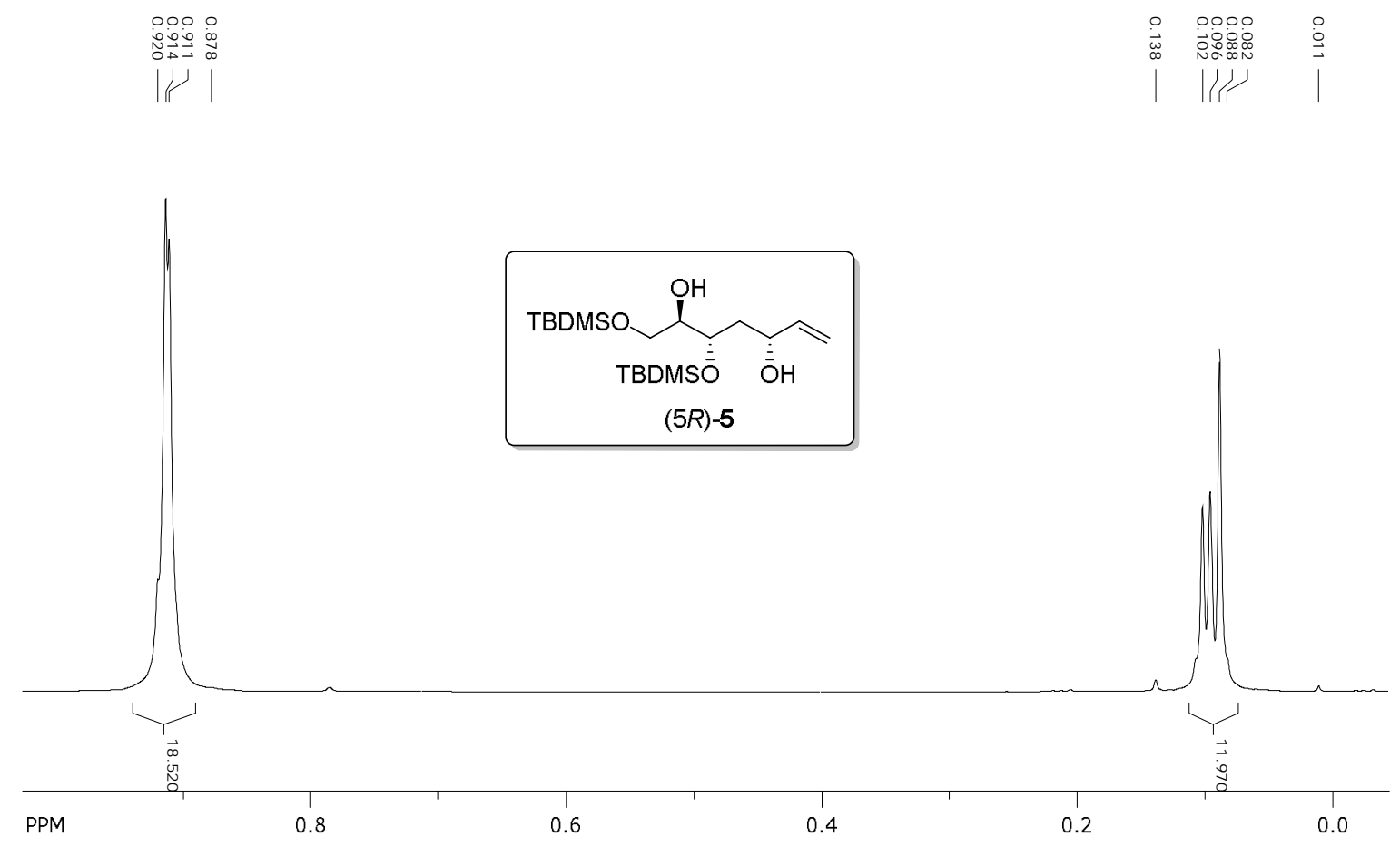

Figure S3. ${ }^{1} \mathrm{H}$ NMR spectrum of compound (5R)-5 (continued).

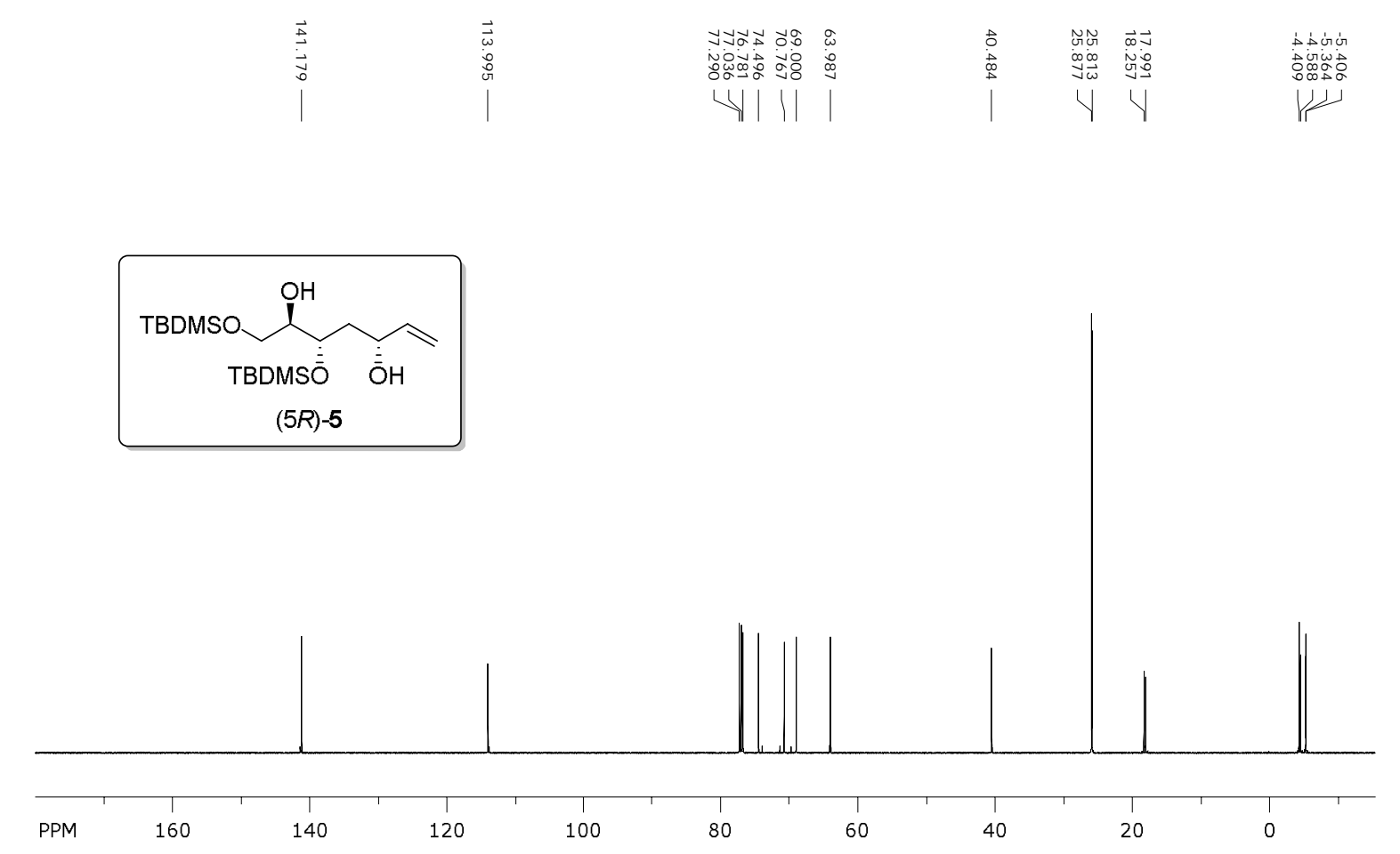

Figure S4. ${ }^{13} \mathrm{C}$ NMR spectrum of compound (5R)-5. 


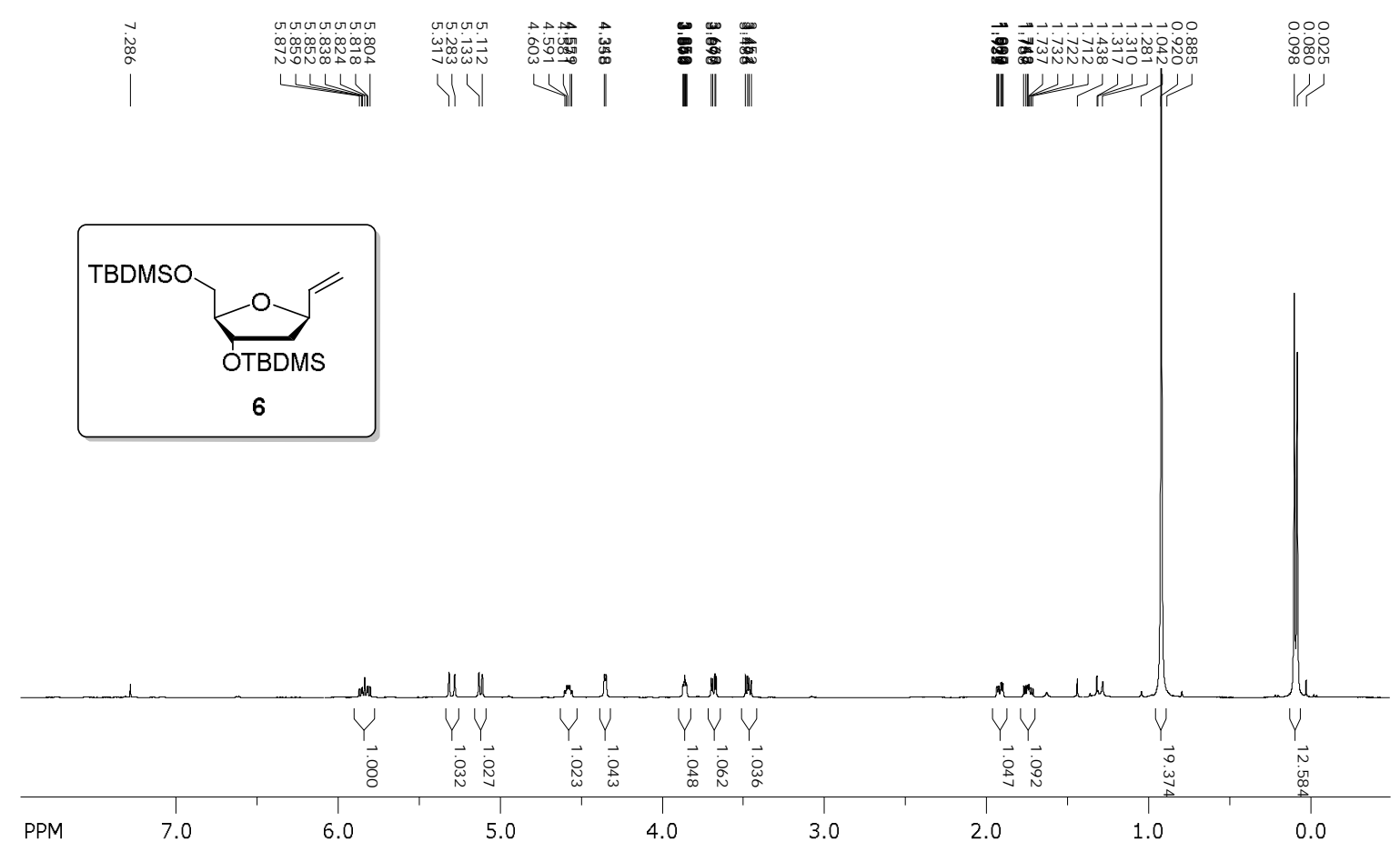

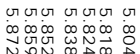

$|\psi||\psi| \mid$
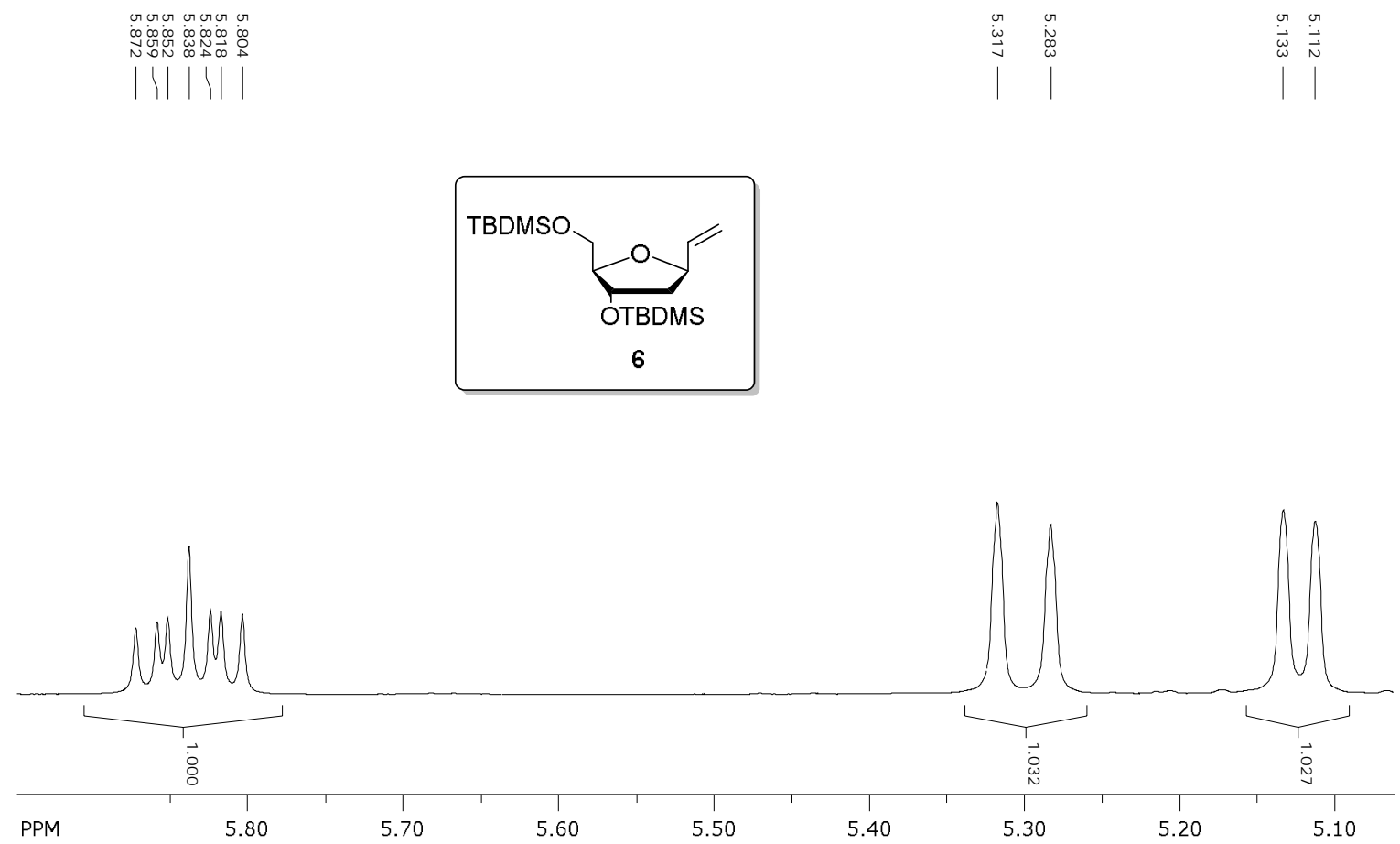

Figure S5. ${ }^{1} \mathrm{H}$ NMR spectrum of compound 6. 

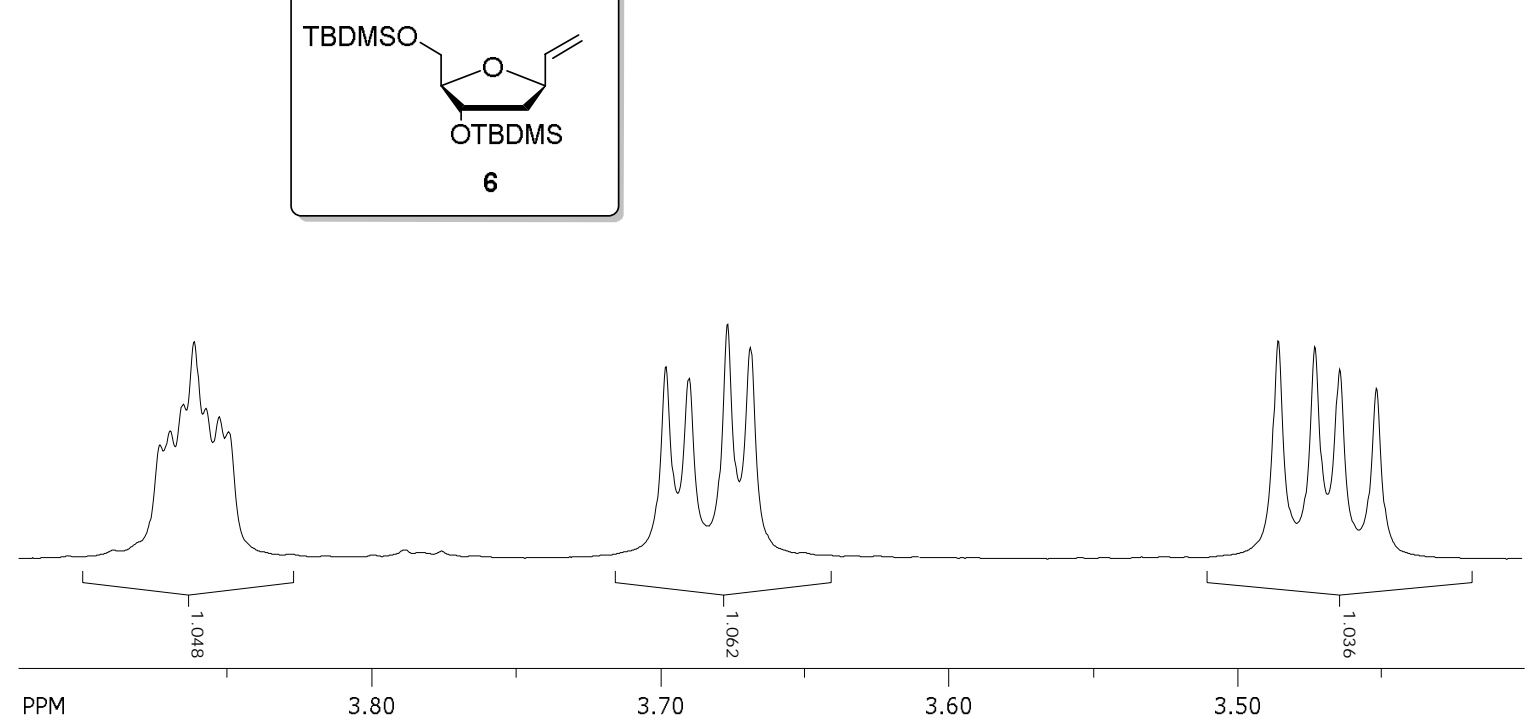

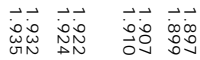

|| || || ||
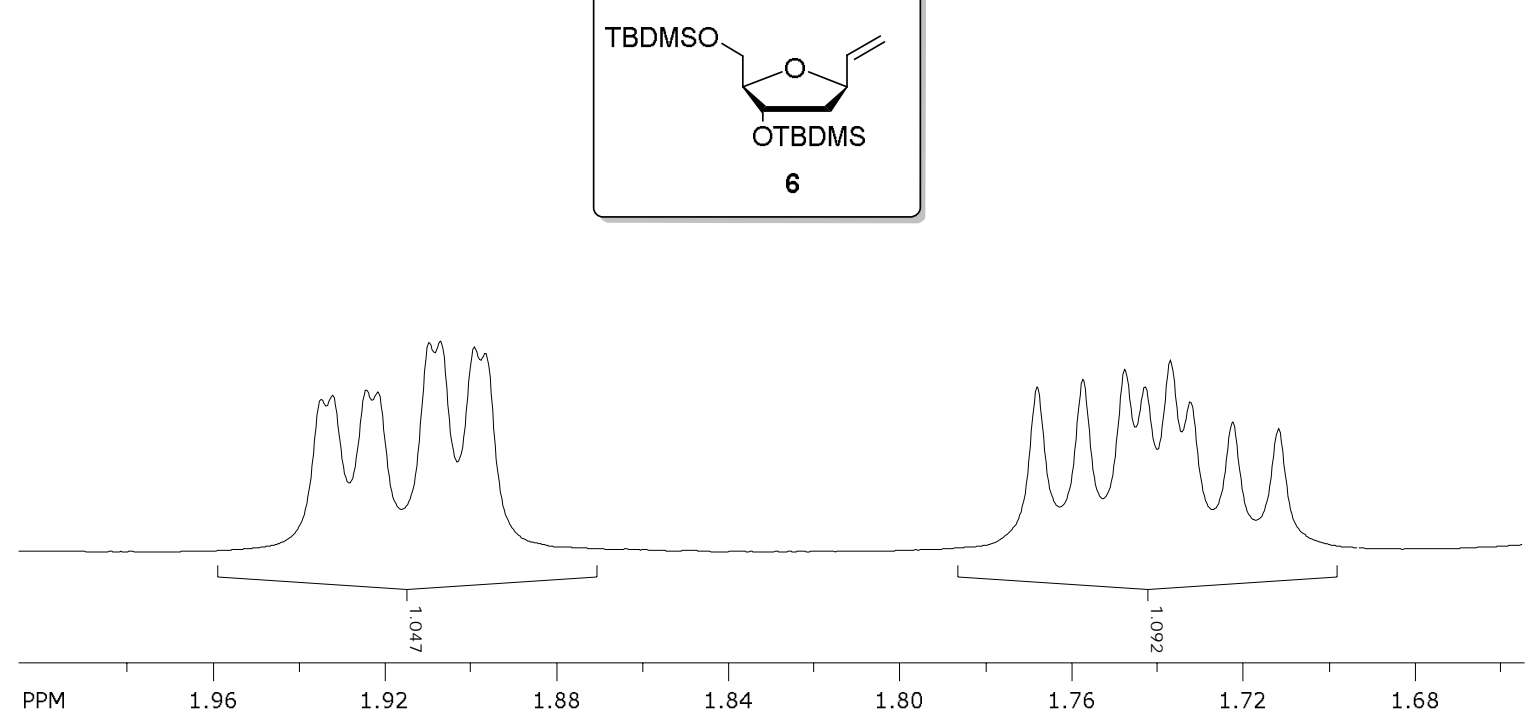

Figure S5. ${ }^{1} \mathrm{H}$ NMR spectrum of compound 6 (continued). 


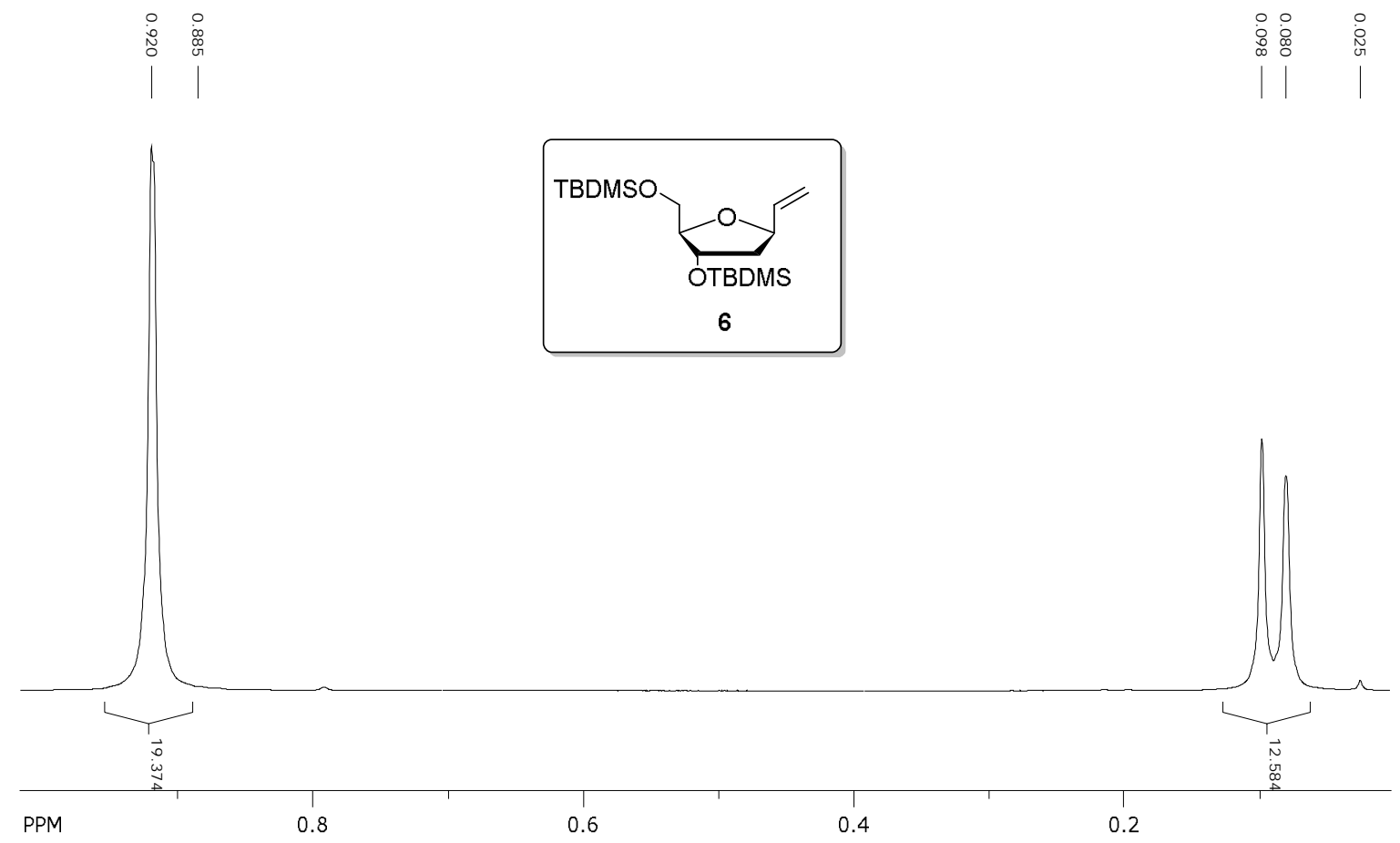

Figure S5. ${ }^{1} \mathrm{H}$ NMR spectrum of compound 6 (continued).

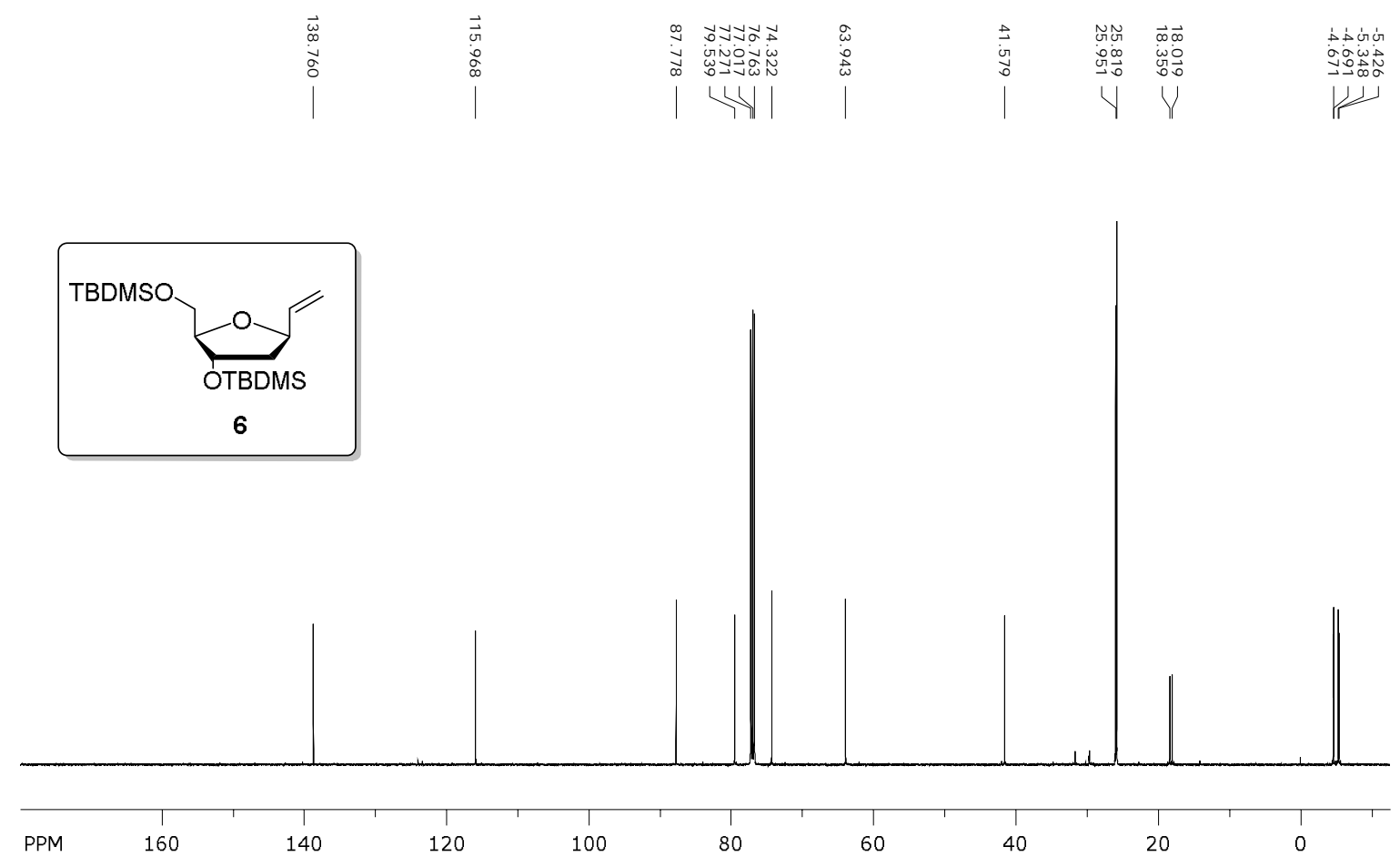

Figure S6. ${ }^{13} \mathrm{C}$ NMR spectrum of compound 6 . 


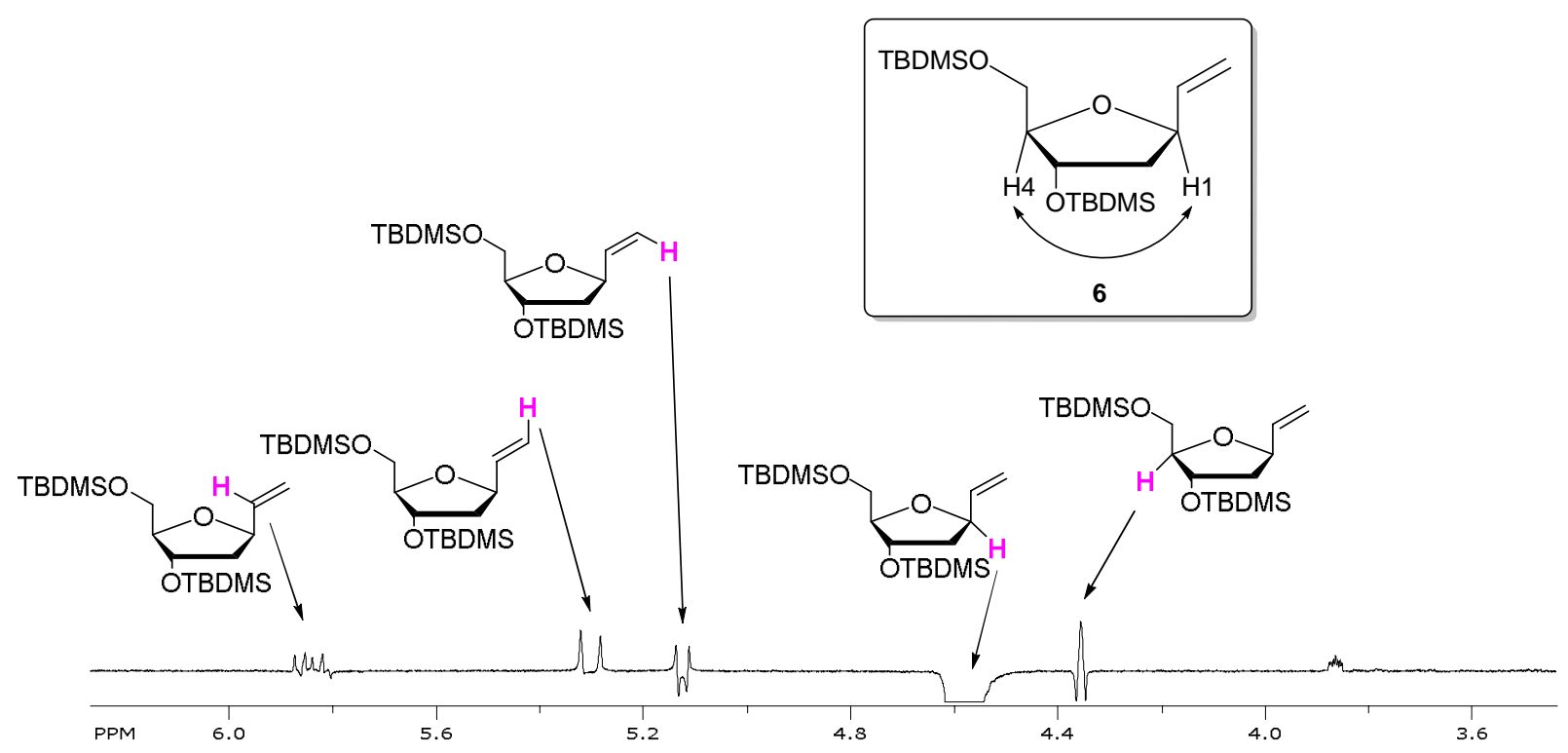

Figure S7. 1D NOESY spectrum of compound 6 with selective excitation of H1.

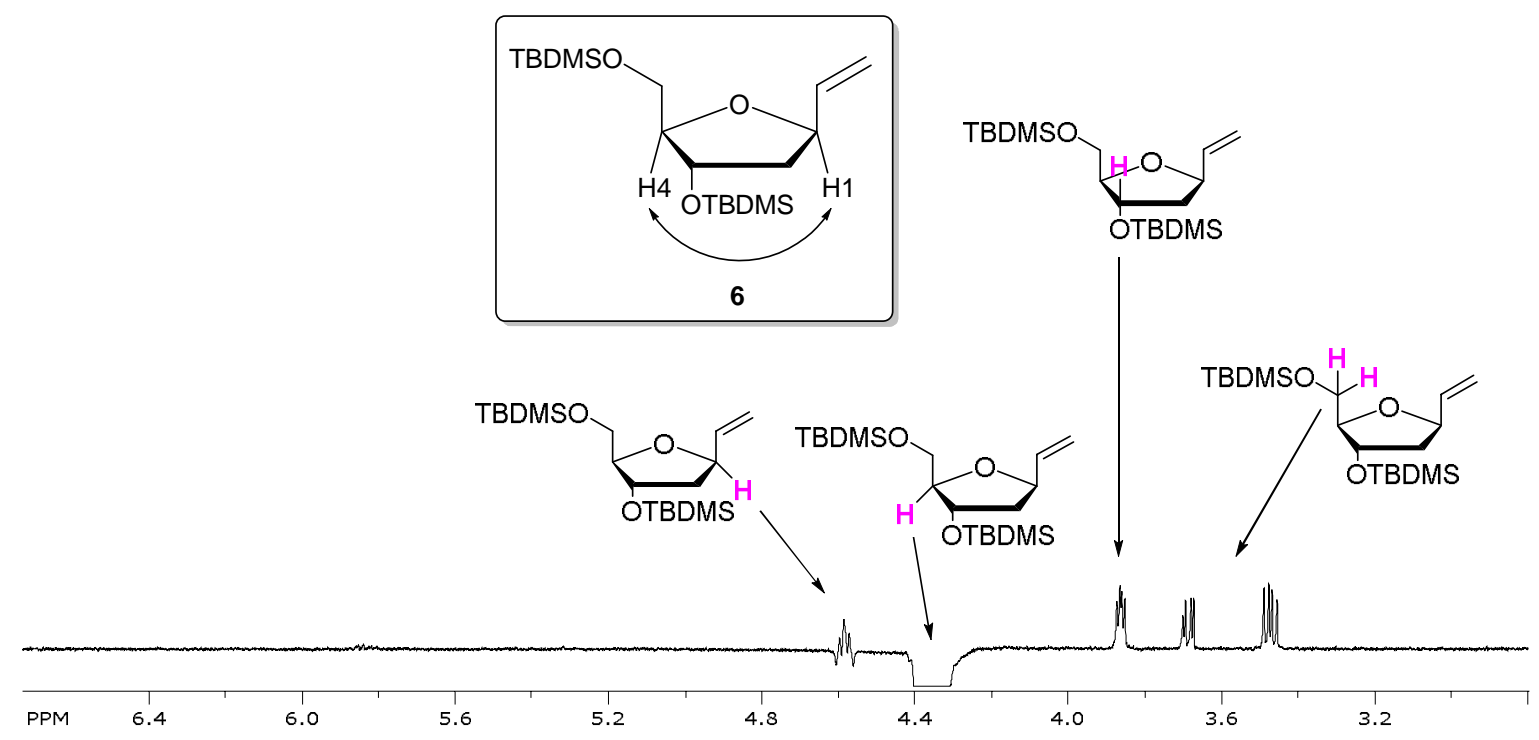

Figure S8. 1D NOESY spectrum of compound 6 with selective excitation of H4. 


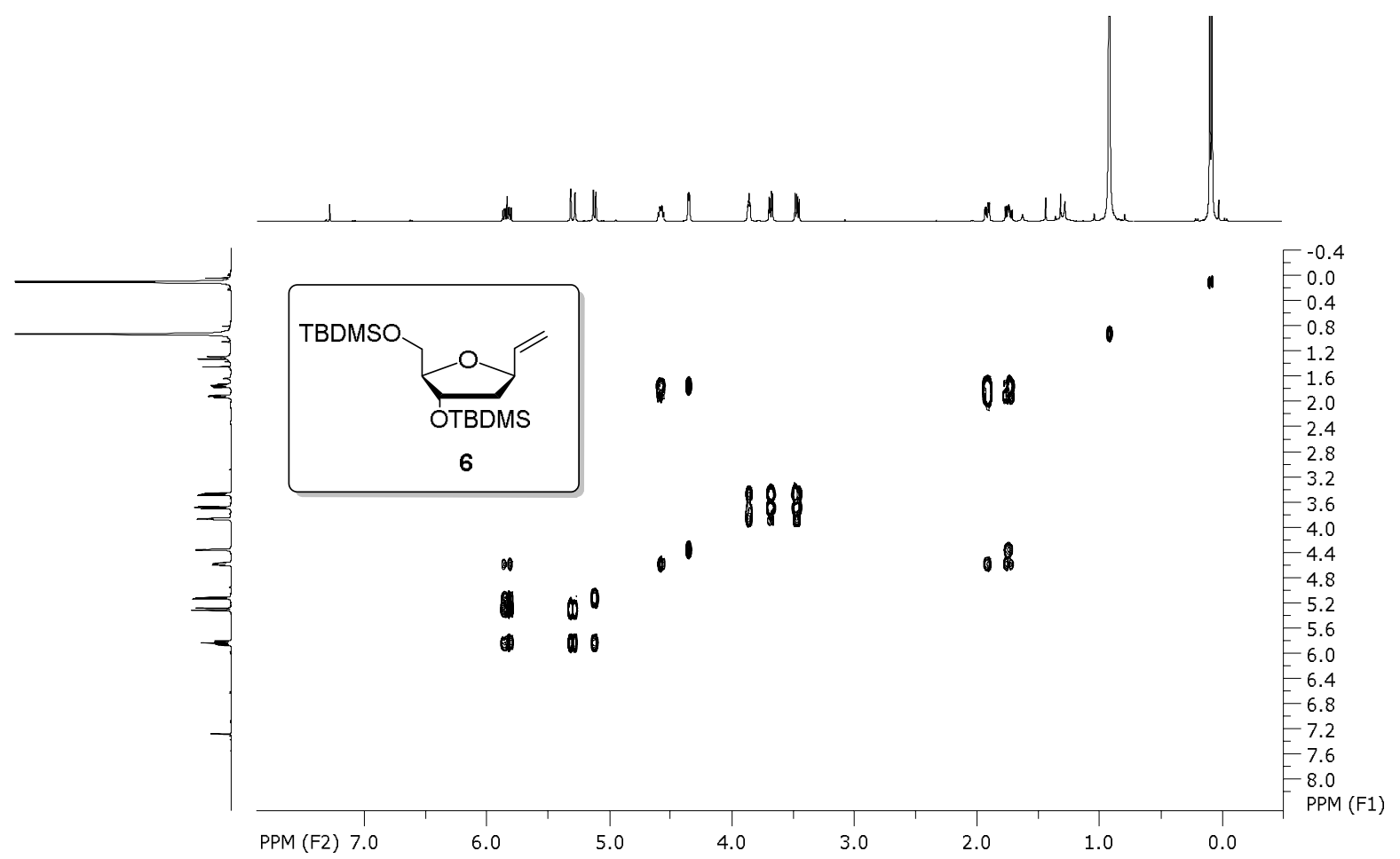

Figure S9. ${ }^{1} \mathrm{H}-{ }^{1} \mathrm{H}$ COSY spectrum of compound 6.

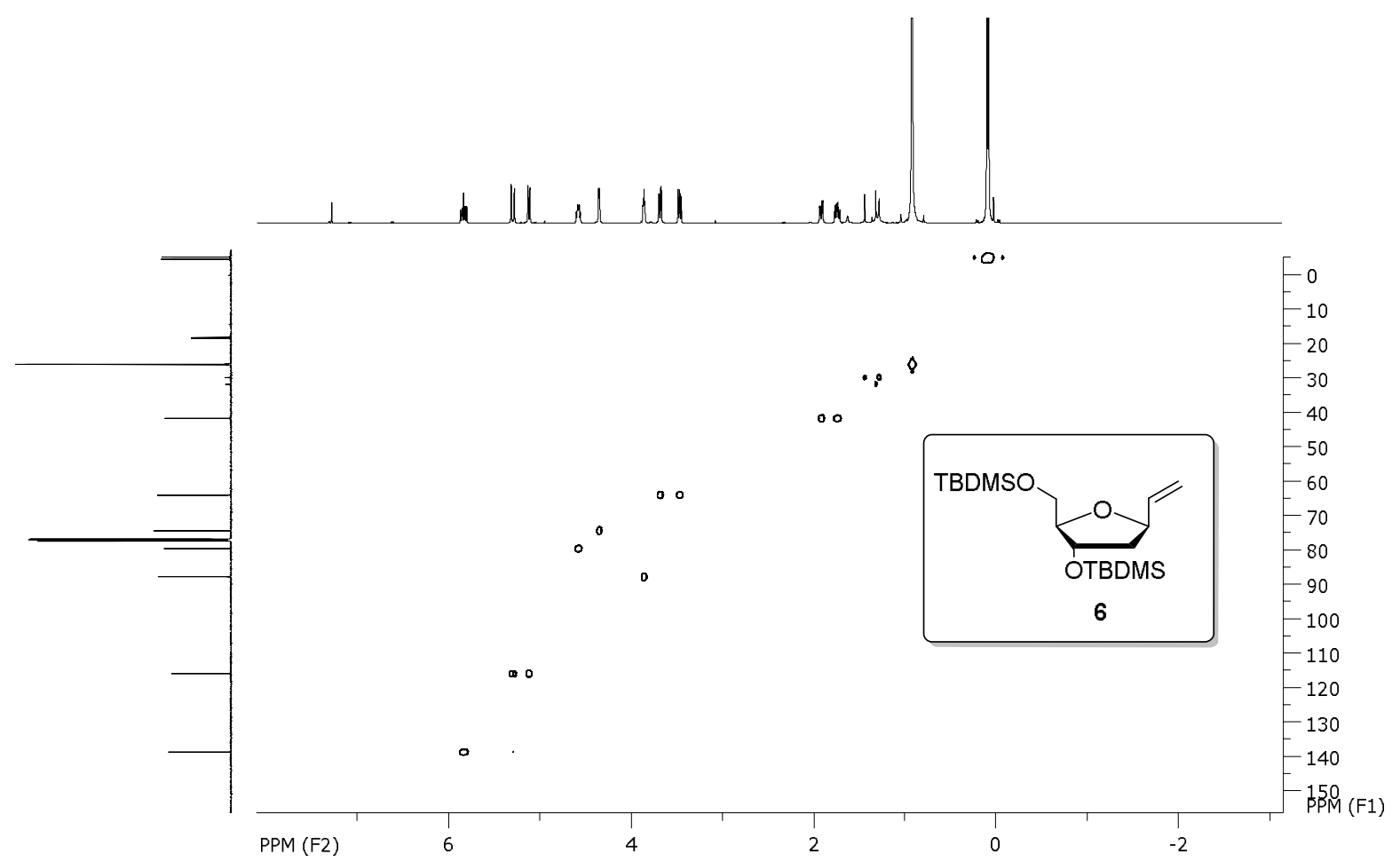

Figure S10. ${ }^{1} \mathrm{H}-{ }^{13} \mathrm{C}$ HSQC spectrum of compound 6. 


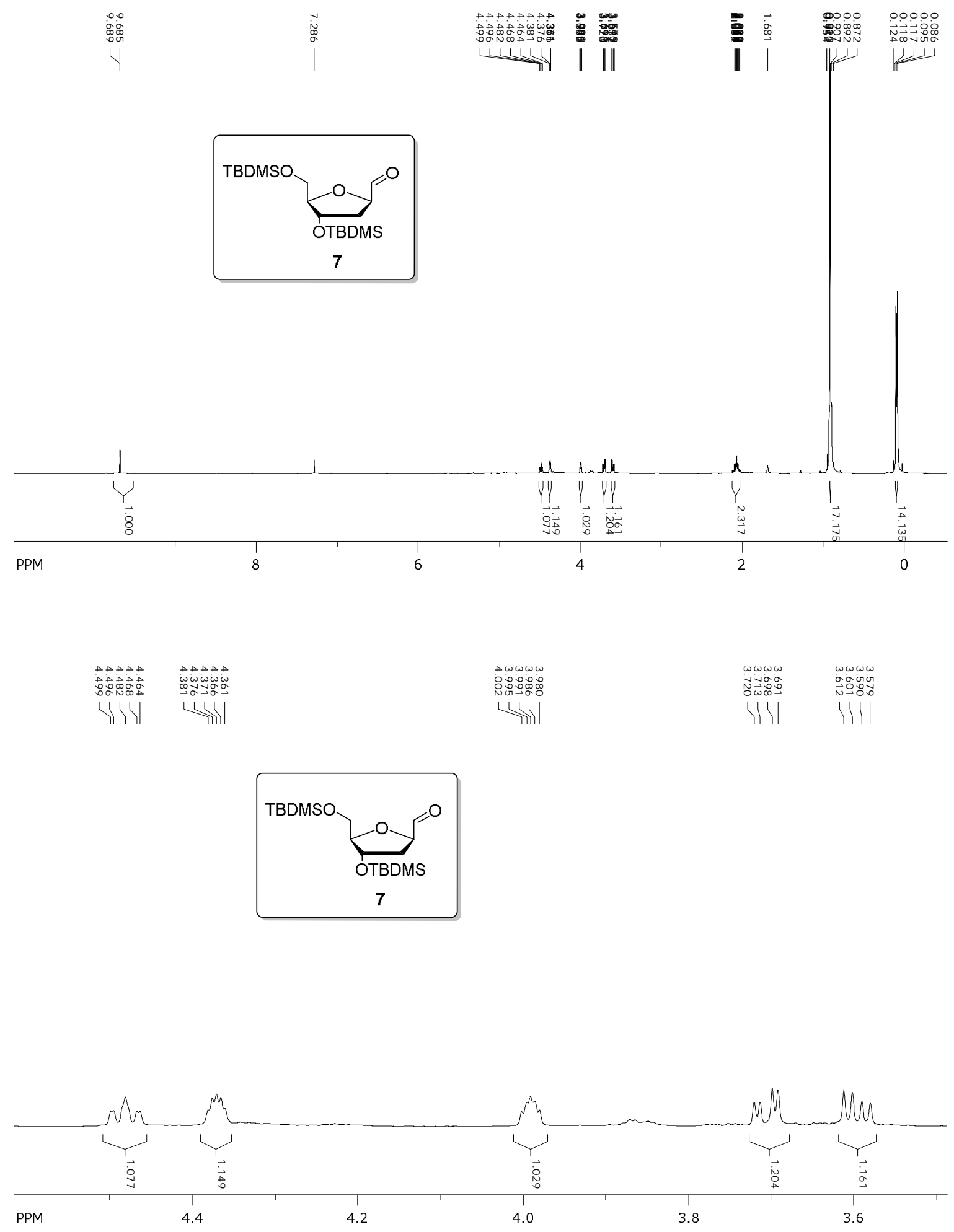

Figure S11. ${ }^{1}$ H NMR spectrum of compound 7. 


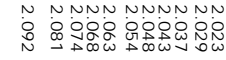

| ||l||||||

葛

(
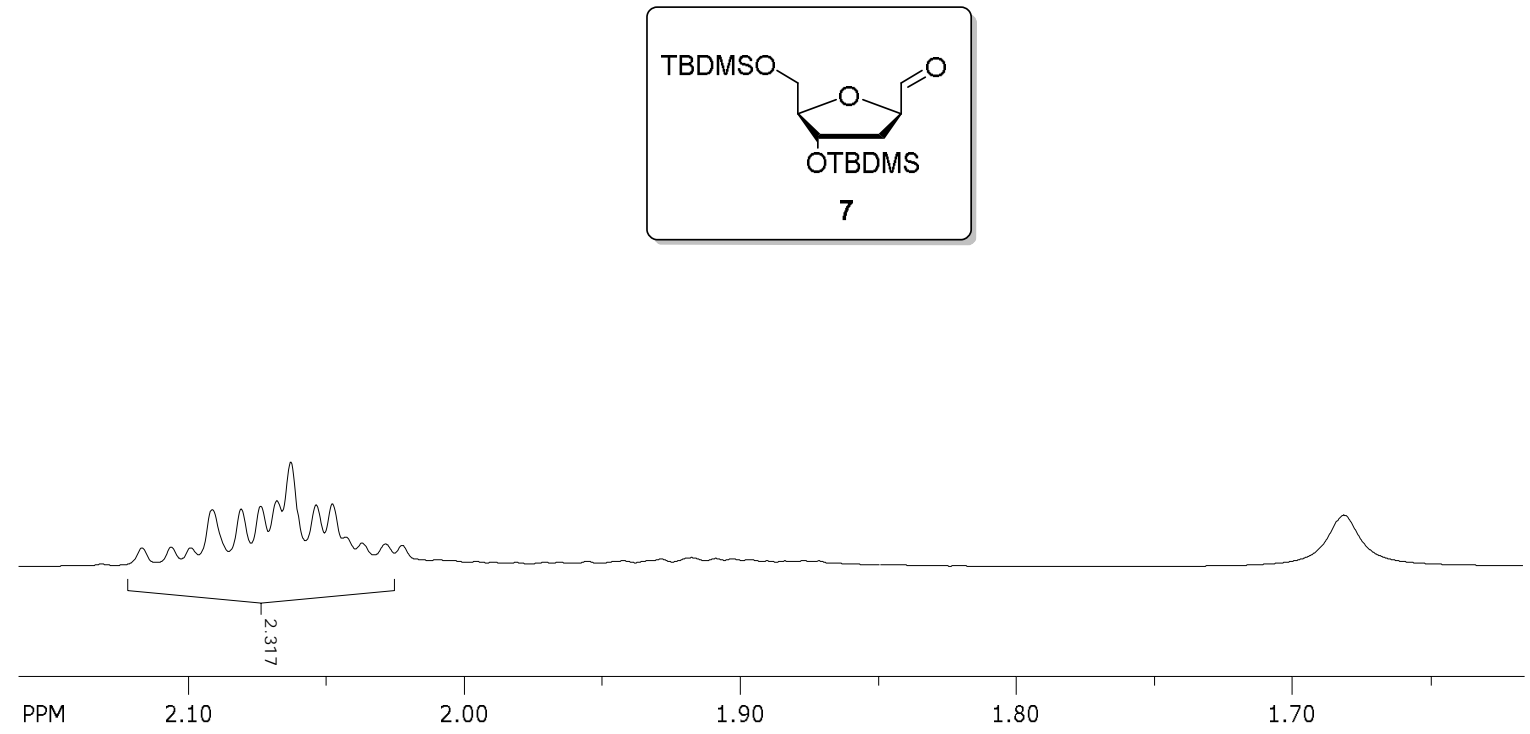

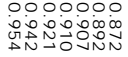

ll |lYl

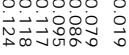

|H/J|

TBDMSO

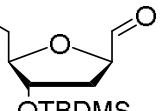

OTBDMS

7

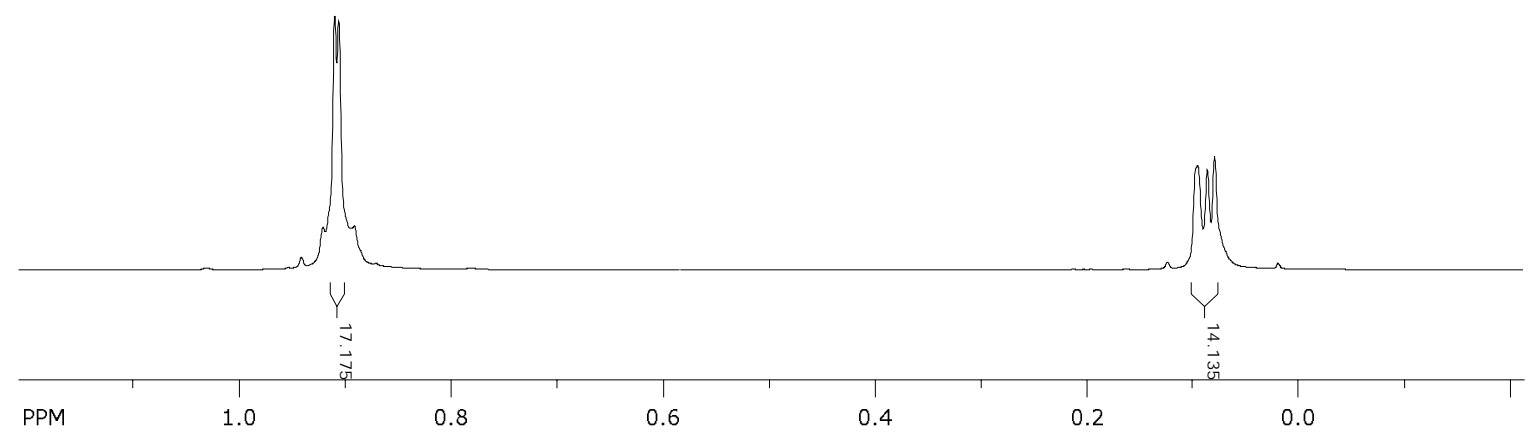

Figure S11. ${ }^{1} \mathrm{H}$ NMR spectrum of compound 7 (continued). 

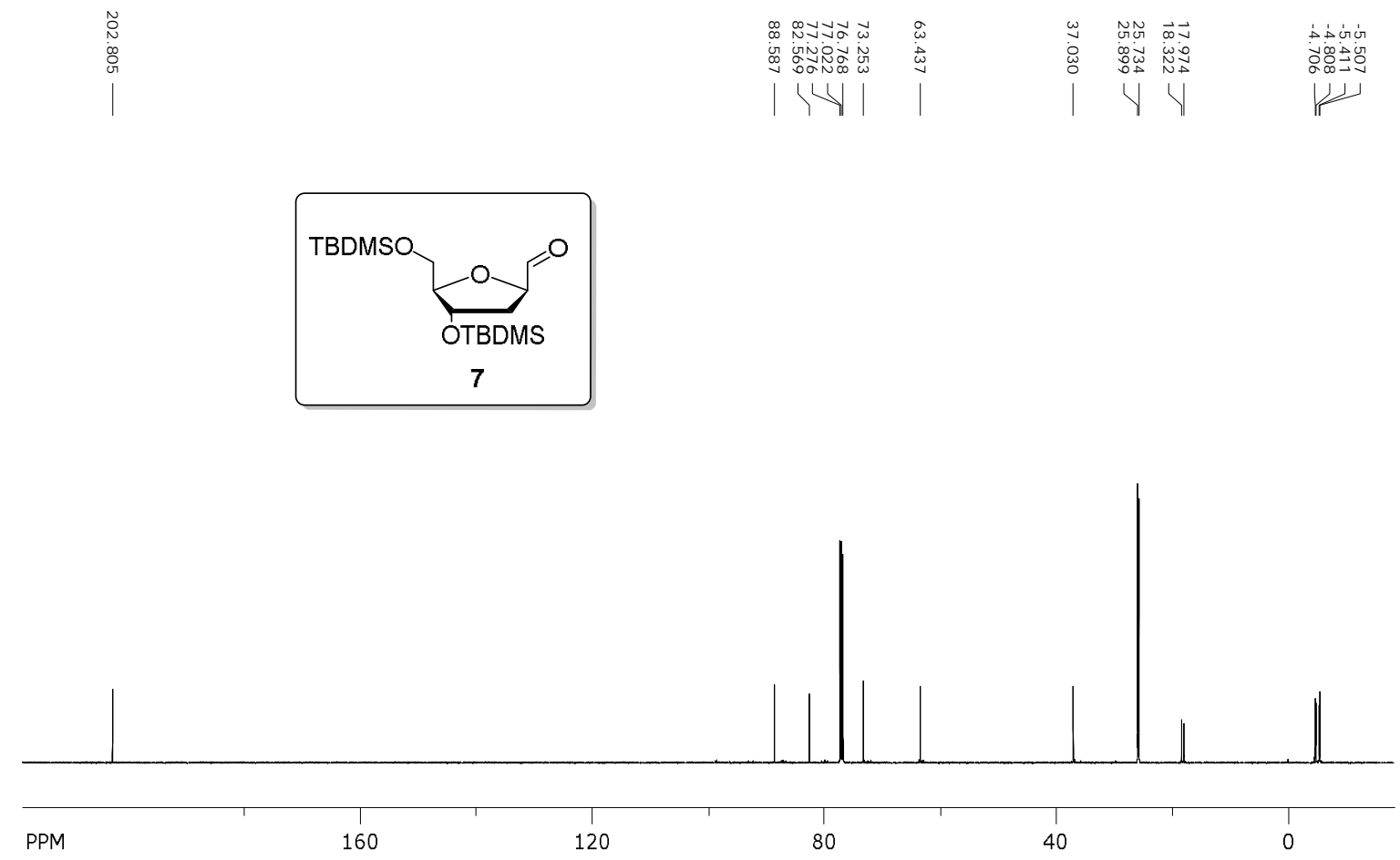

Figure S12. ${ }^{13} \mathrm{C}$ NMR spectrum of compound 7.

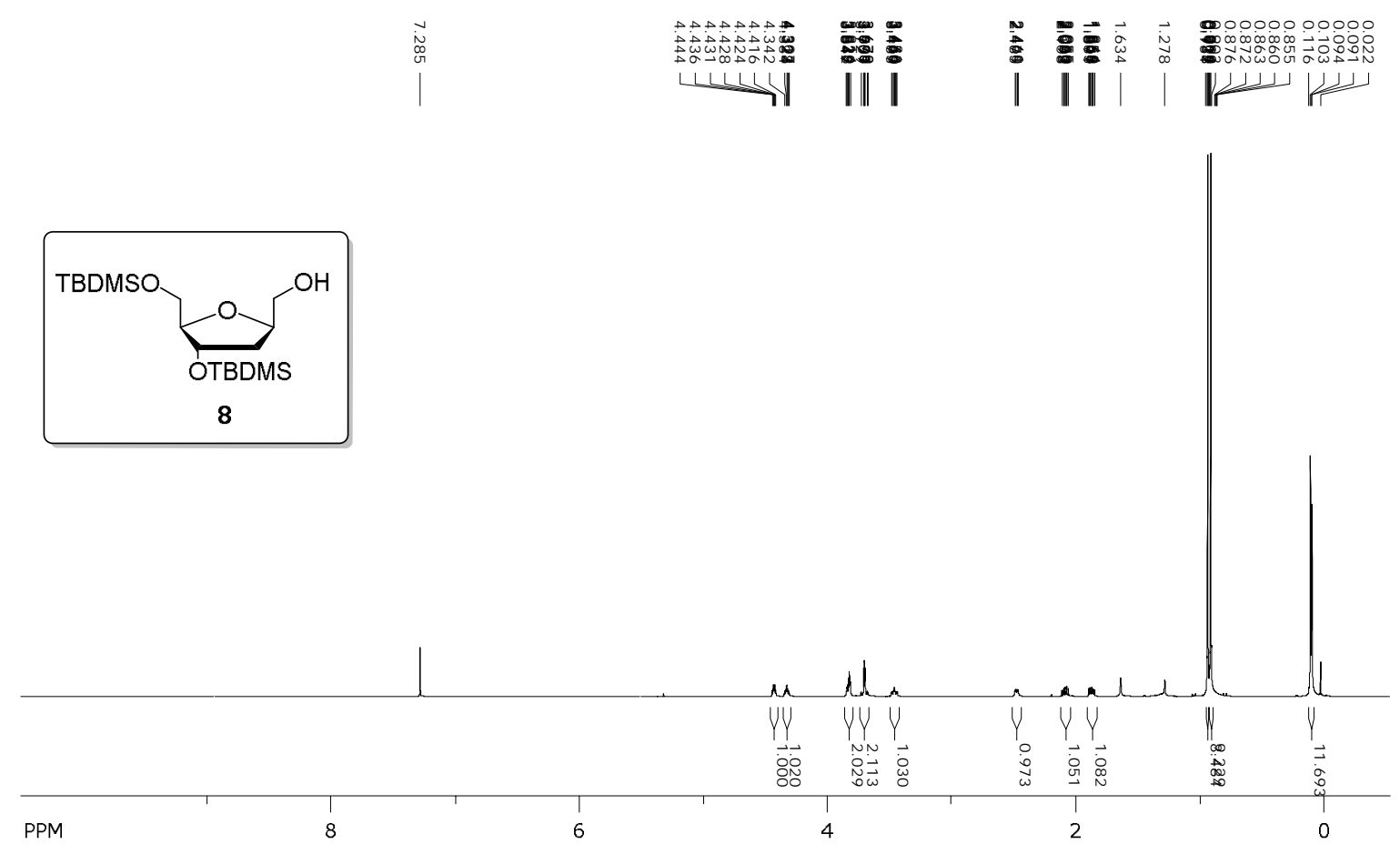

Figure S13. ${ }^{1}$ H NMR spectrum of compound 8. 

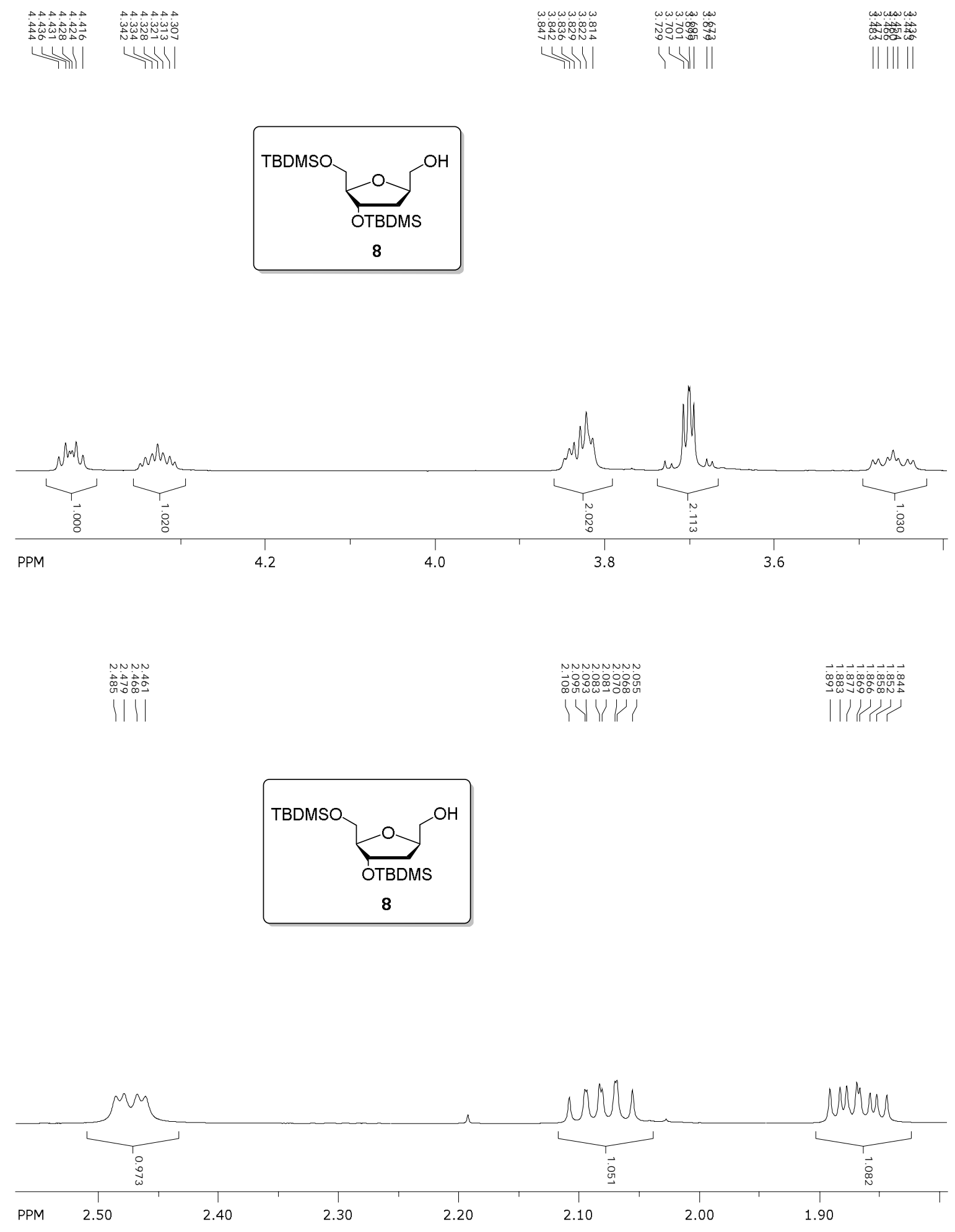

Figure S13. ${ }^{1} \mathrm{H}$ NMR spectrum of compound 8 (continued). 


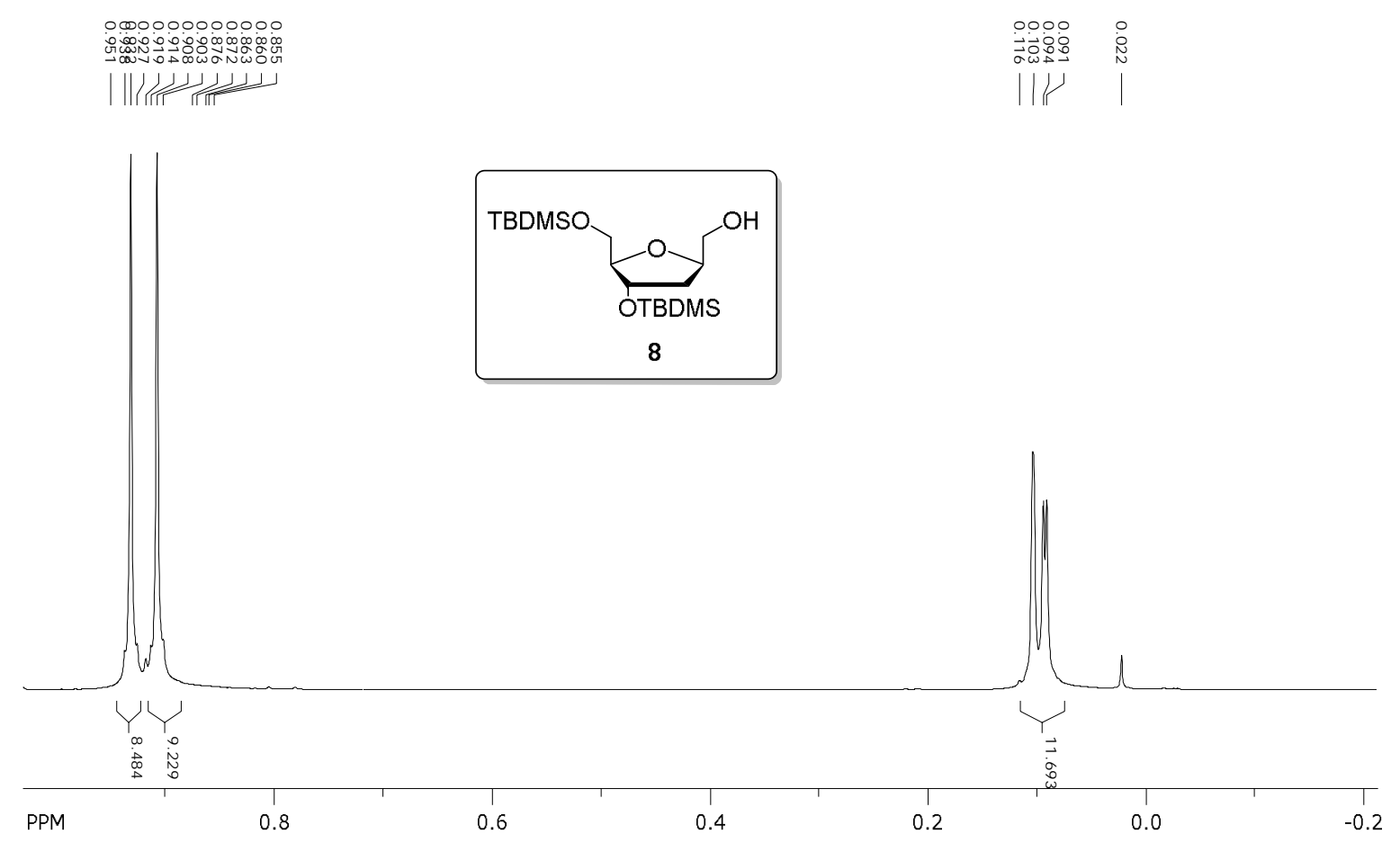

Figure S13. ${ }^{1} \mathrm{H}$ NMR spectrum of compound 8 (continued).

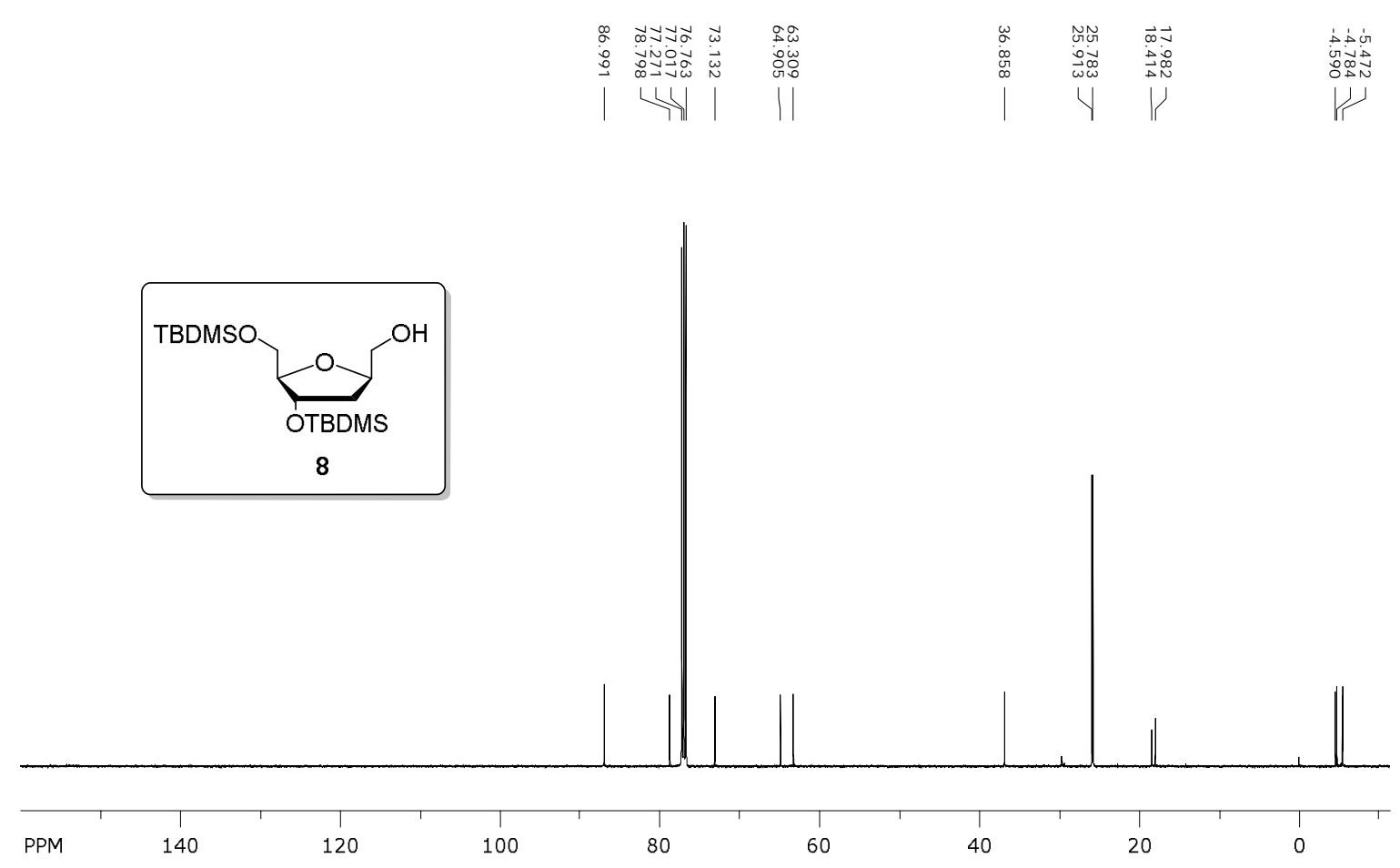

Figure S14. ${ }^{13} \mathrm{C}$ NMR spectrum of compound 8 . 


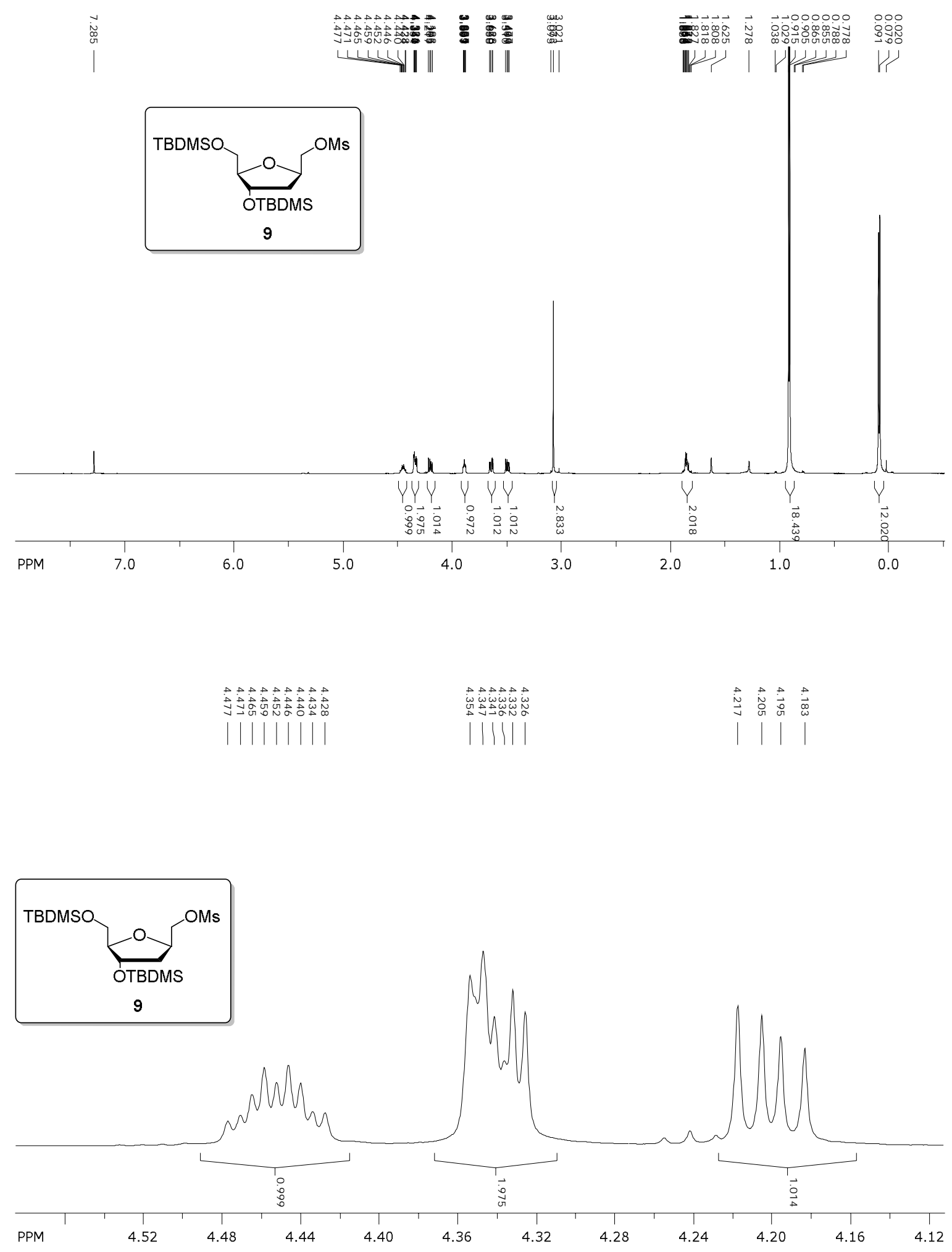

Figure S15. ${ }^{1} \mathrm{H}$ NMR spectrum of compound 9. 


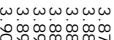

$41 Y 1114$
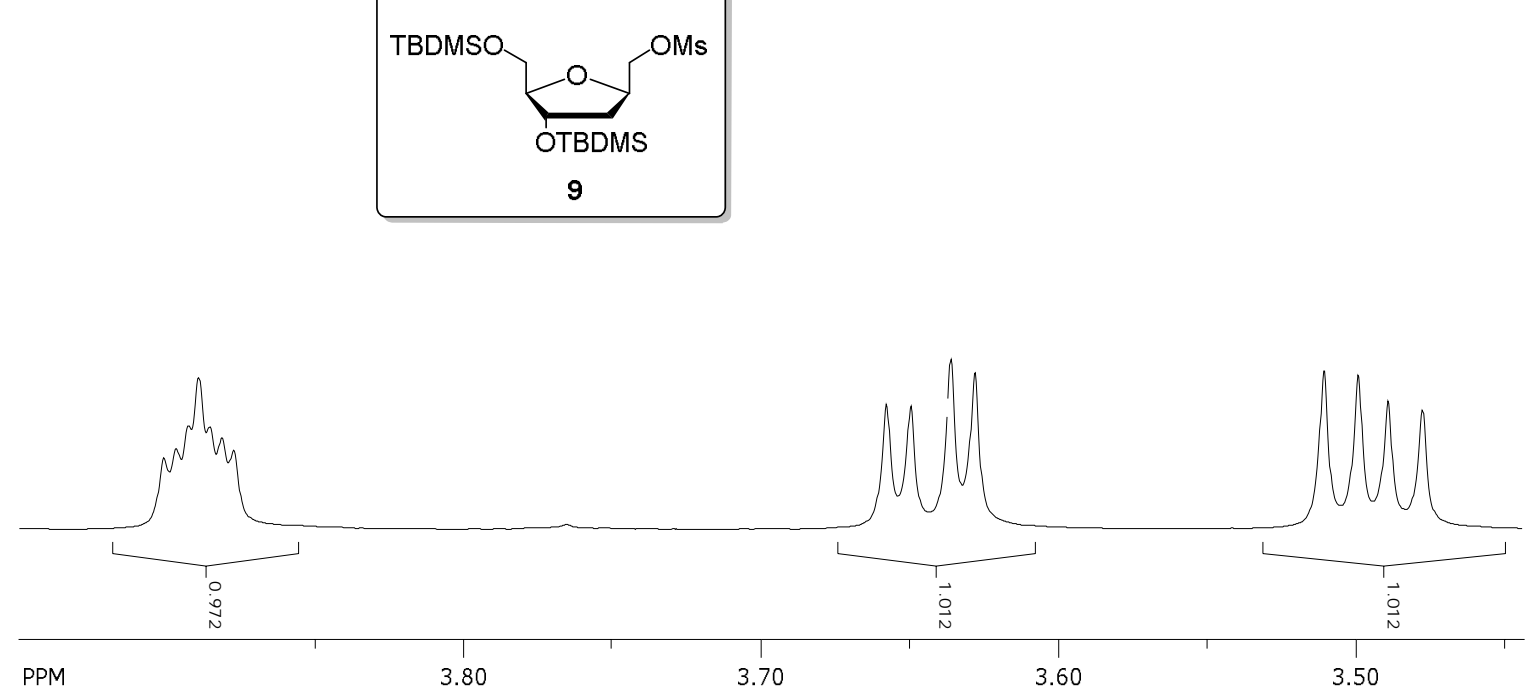

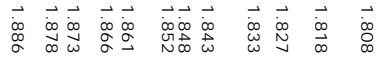

| || || ||| || | |
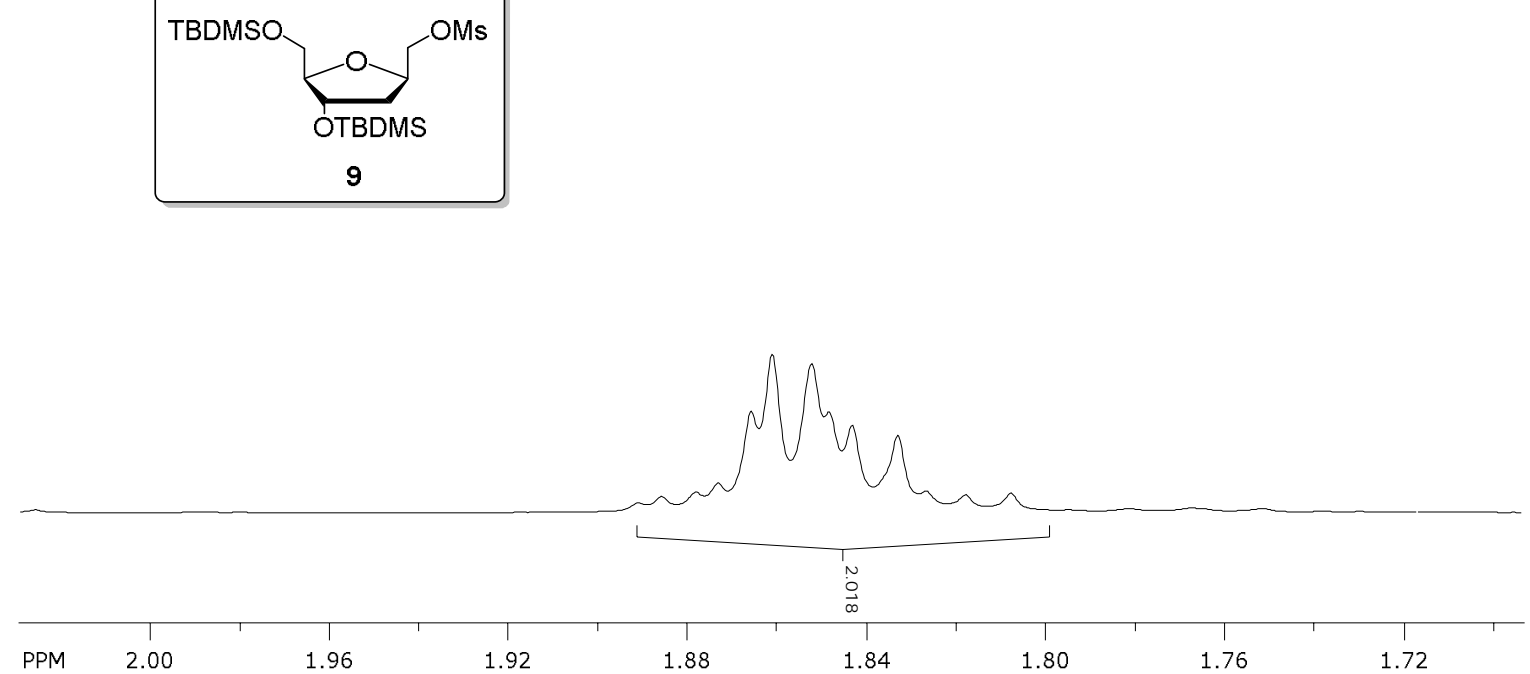

Figure S15. ${ }^{1} \mathrm{H}$ NMR spectrum of compound 9 (continued). 


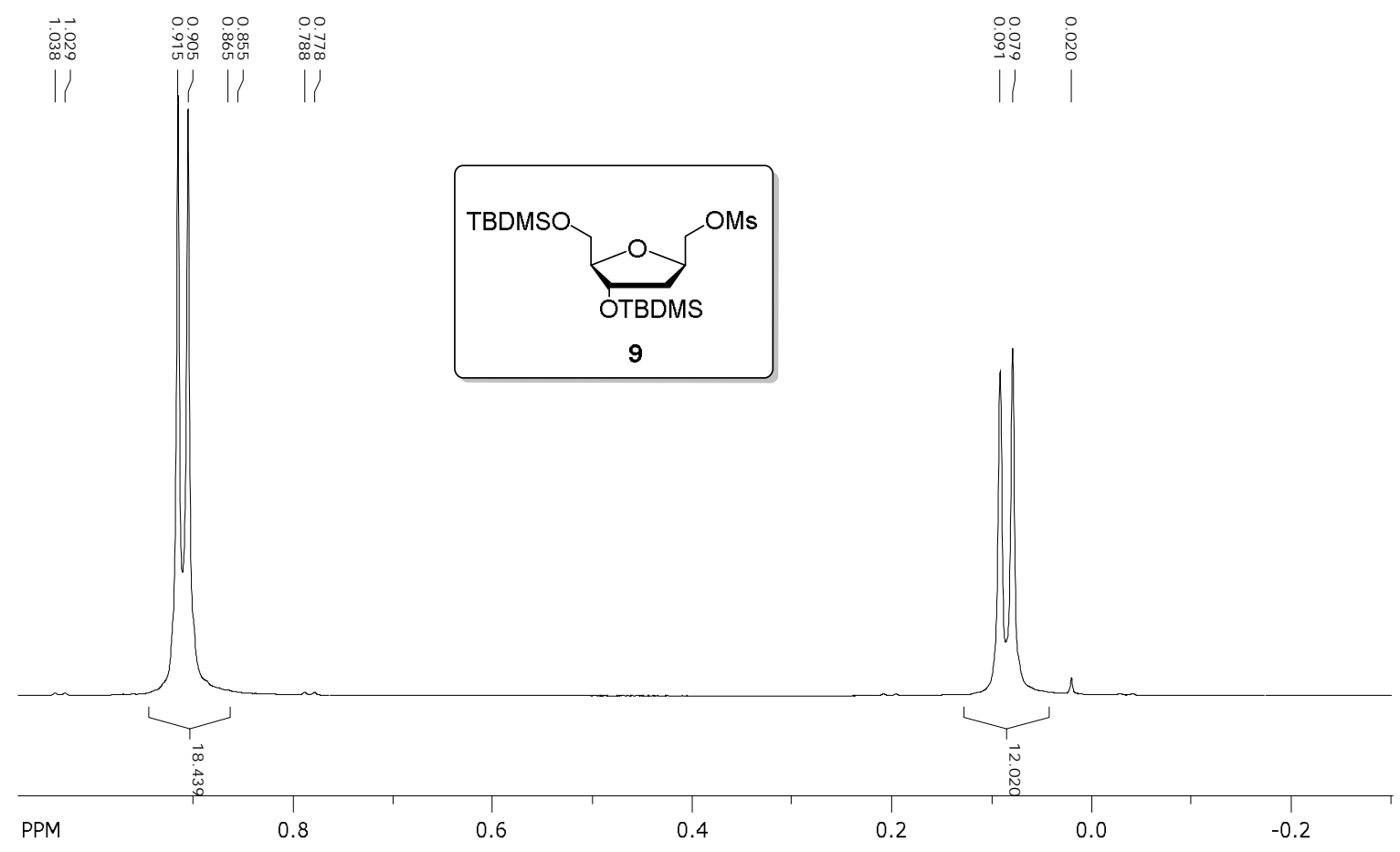

Figure S15. ${ }^{1} \mathrm{H}$ NMR spectrum of compound 9 (continued).

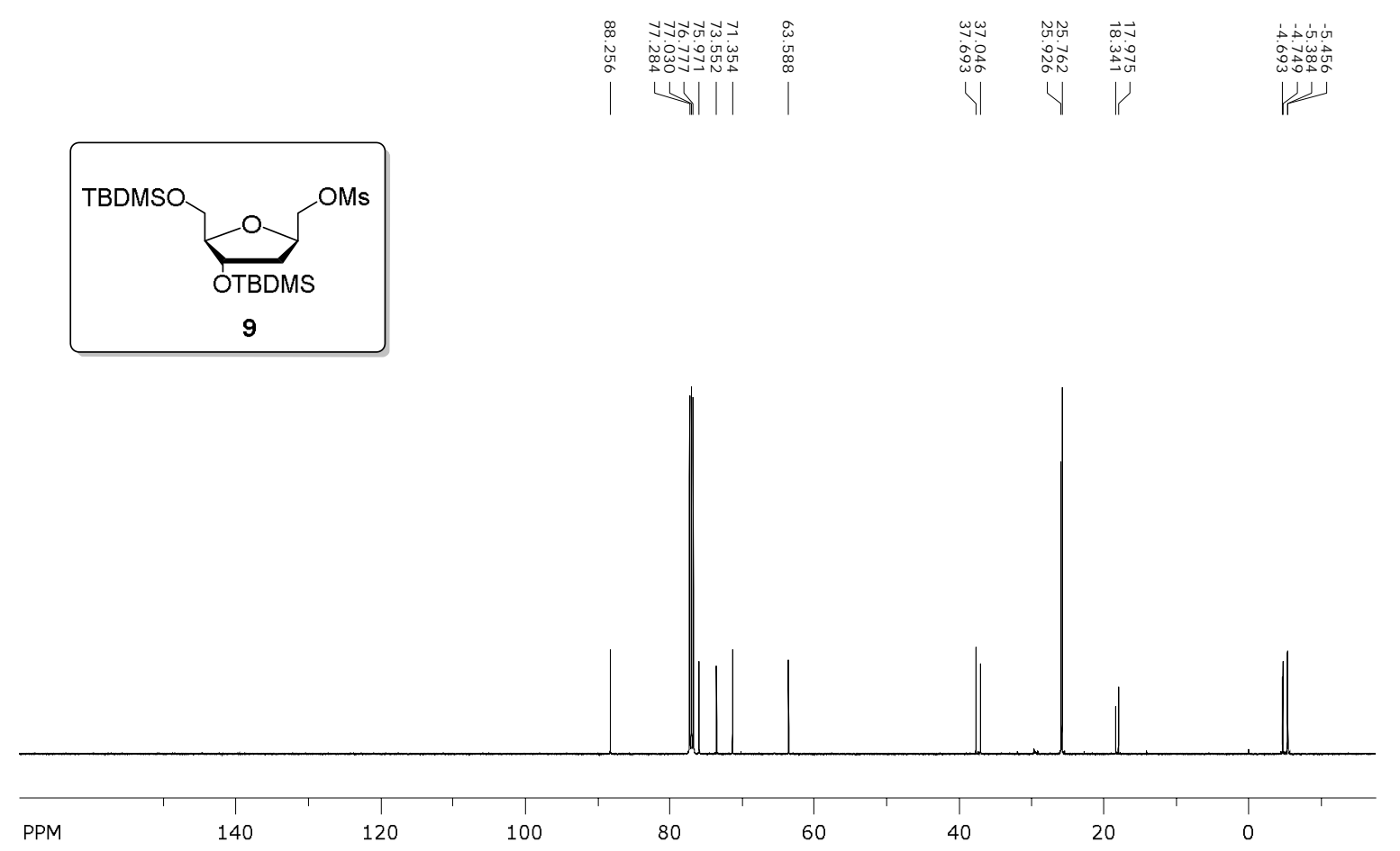

Figure S16. ${ }^{13} \mathrm{C}$ NMR spectrum of compound 9. 


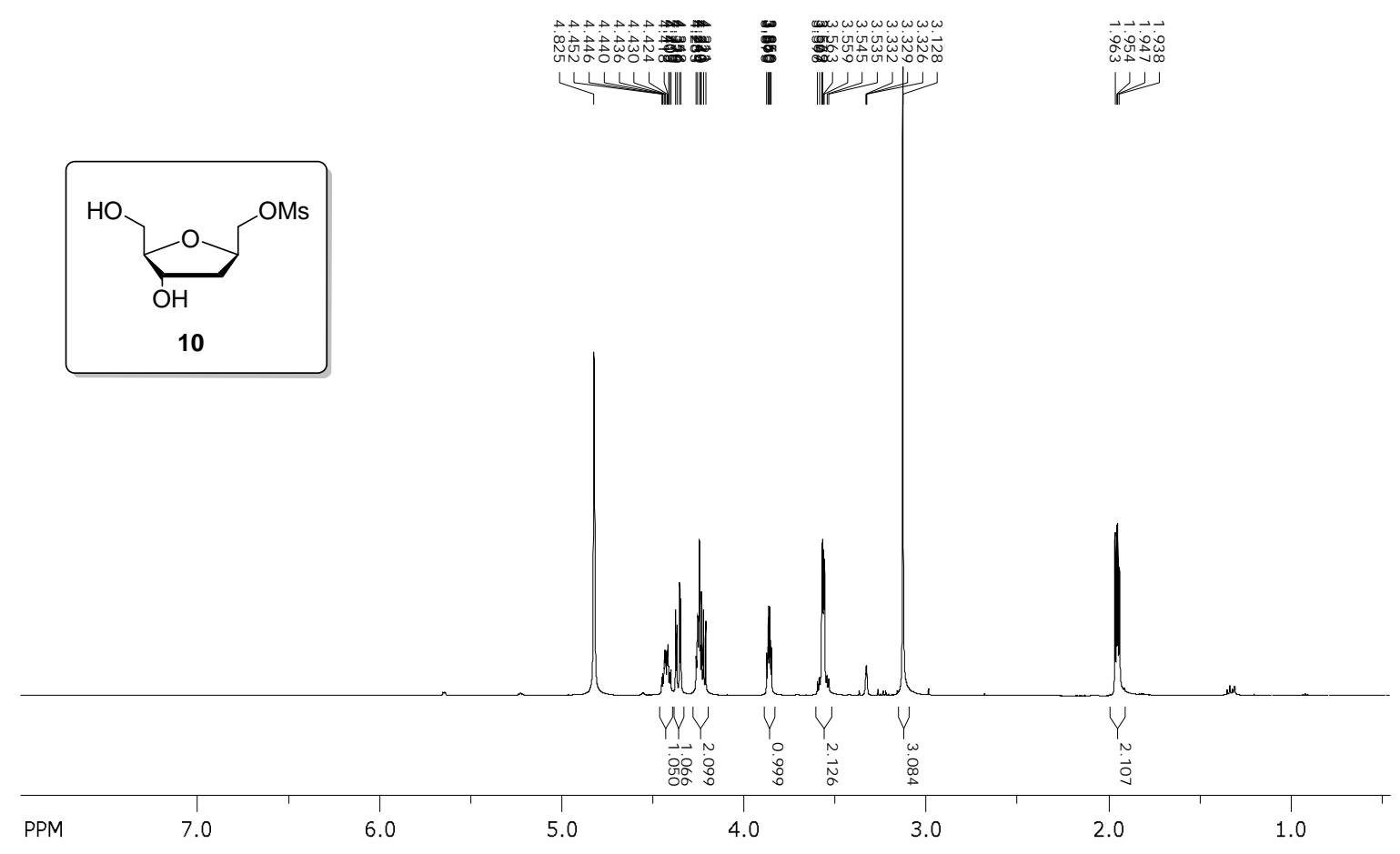

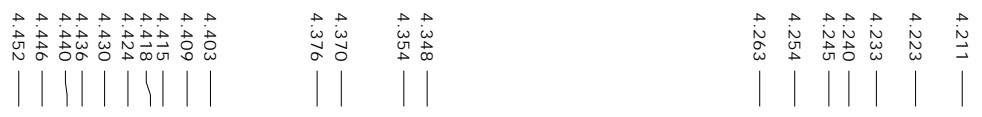
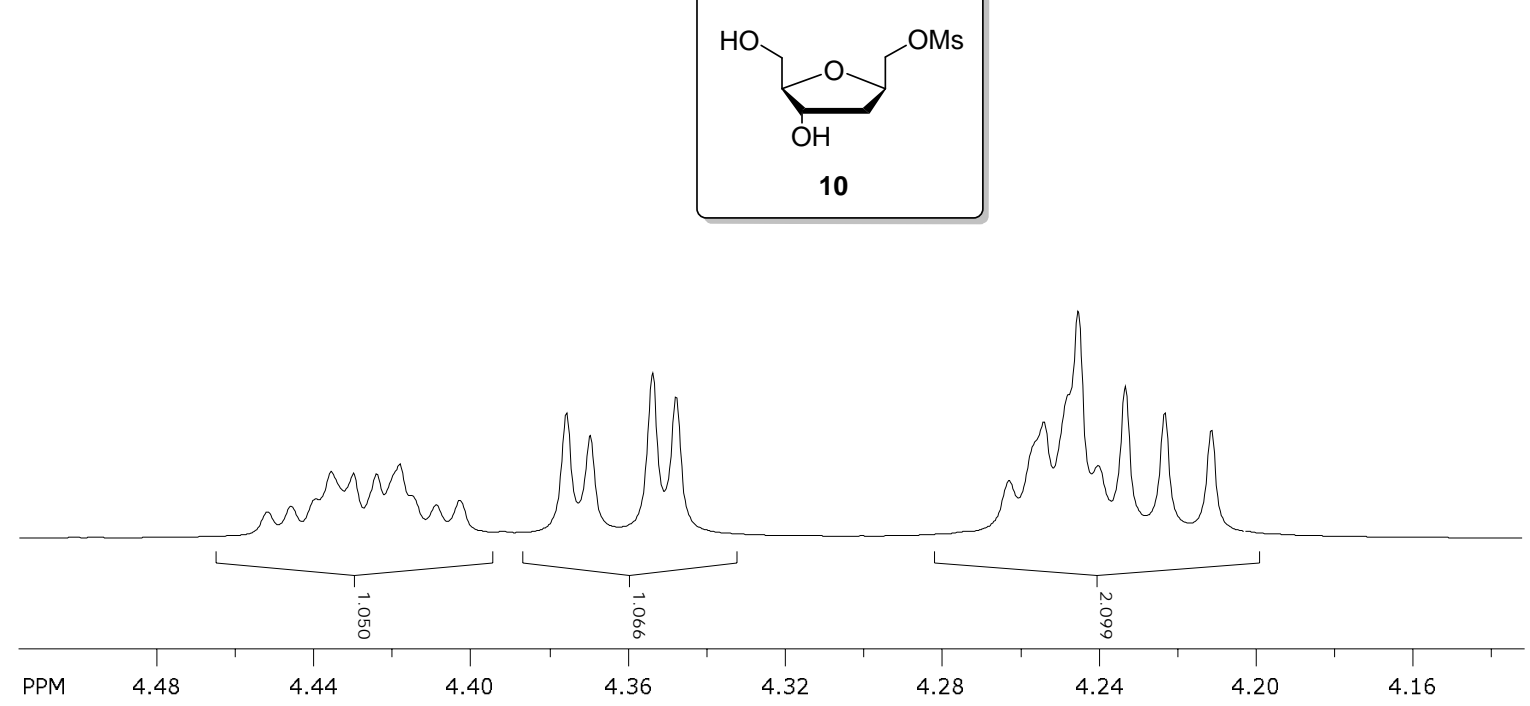

Figure S17. ${ }^{1} \mathrm{H}$ NMR spectrum of compound $\mathbf{1 0}$. 


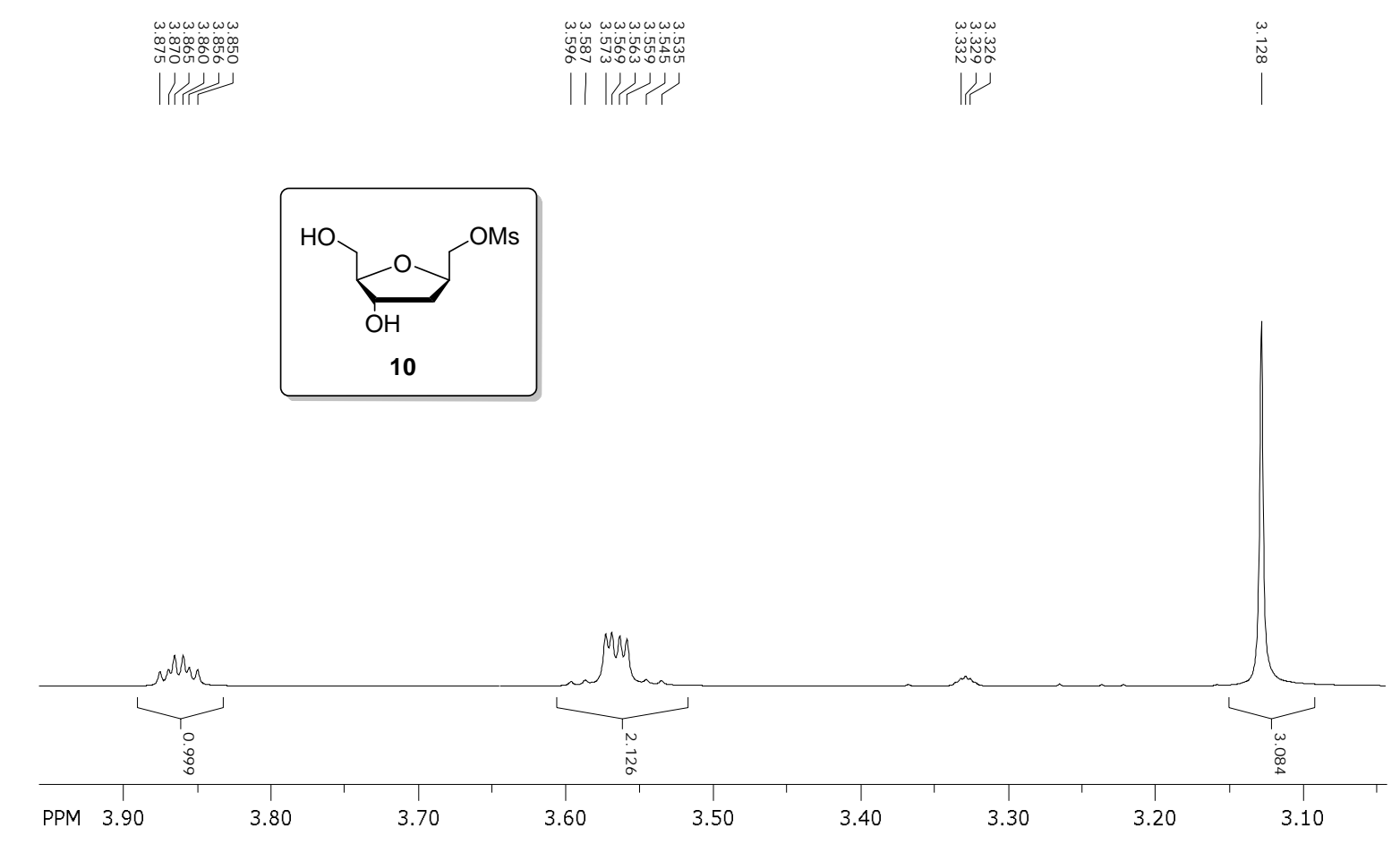

Figure S17. ${ }^{1} \mathrm{H}$ NMR spectrum of compound $\mathbf{1 0}$ (continued).

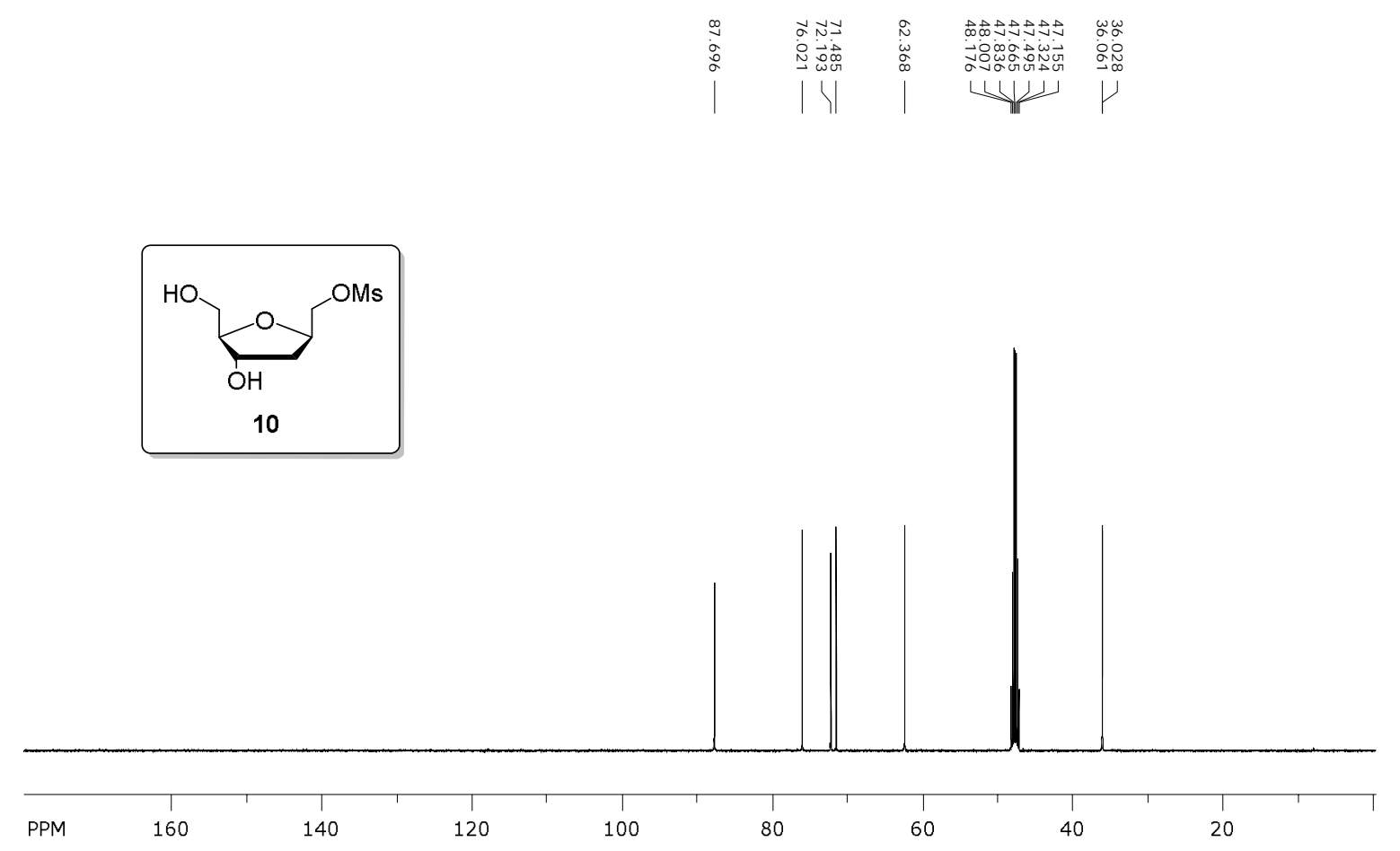

Figure S18. ${ }^{13} \mathrm{C}$ NMR spectrum of compound $\mathbf{1 0}$. 


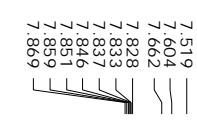

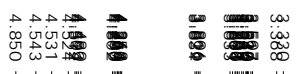 \\ | $\mid$ || || | ||| ||}
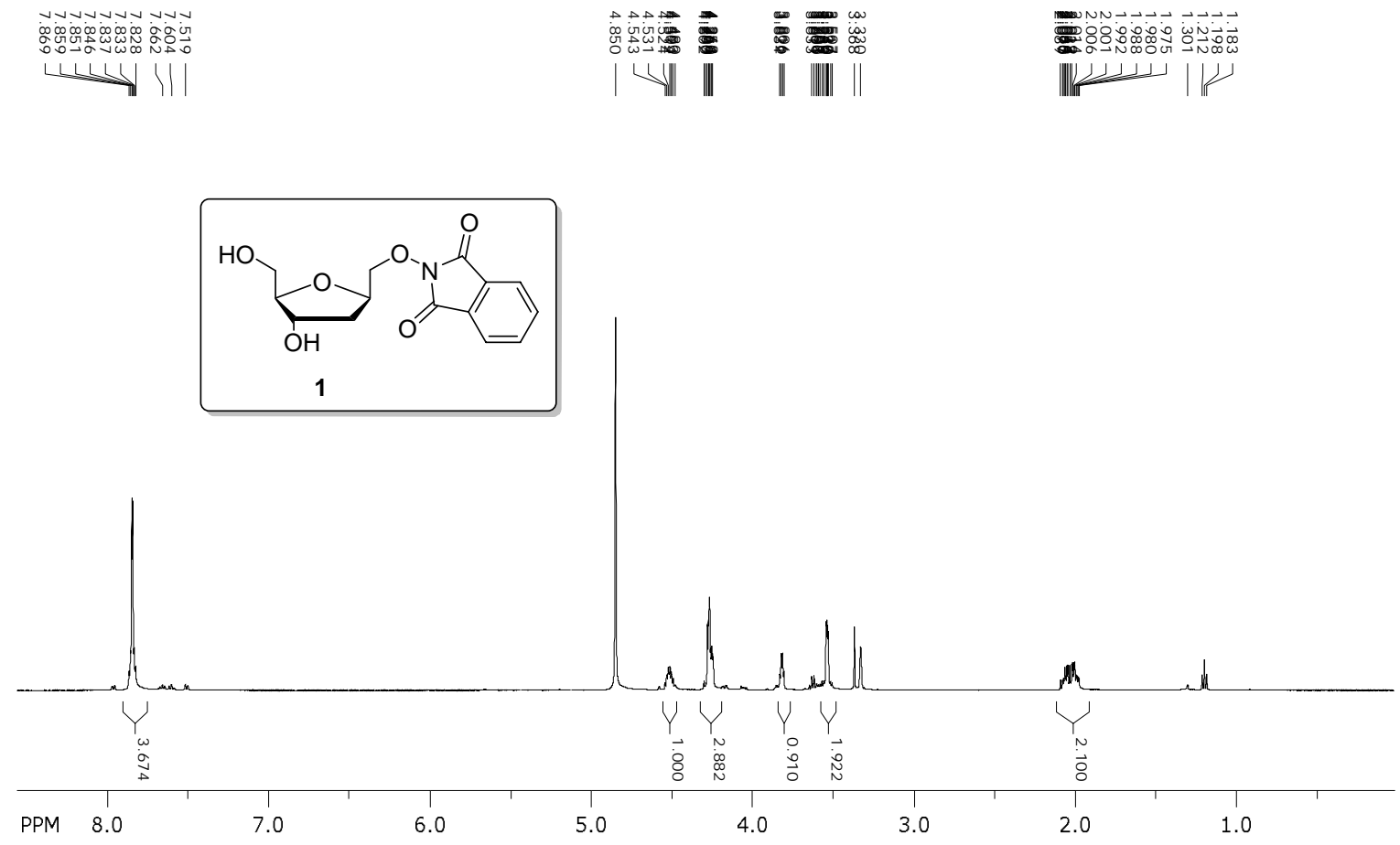

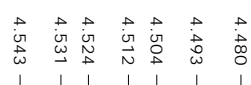

ए। ए।
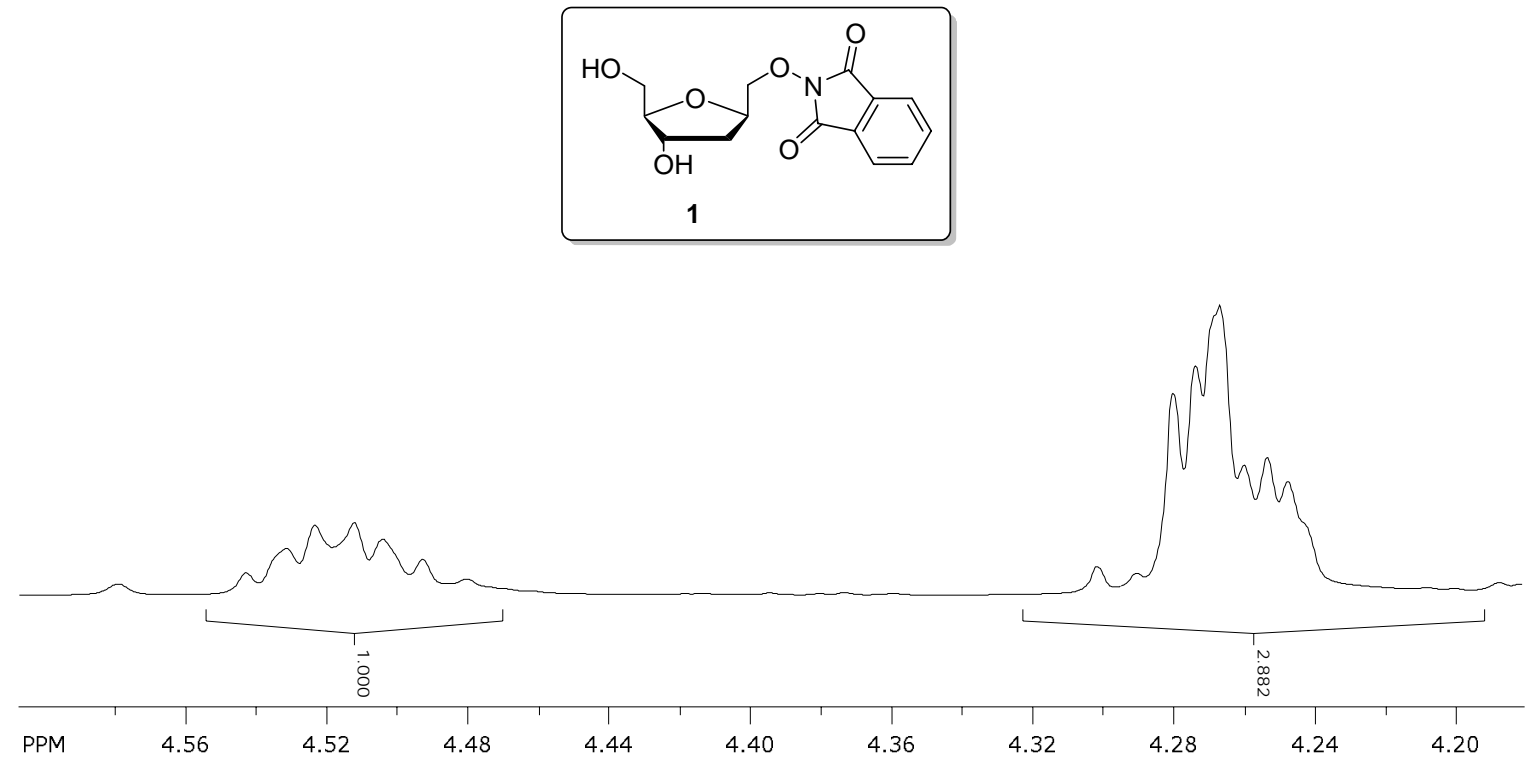

Figure S19. ${ }^{1} \mathrm{H}$ NMR spectrum of compound 1 . 


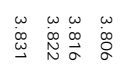

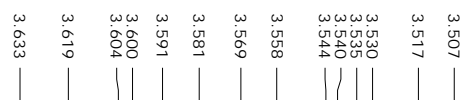
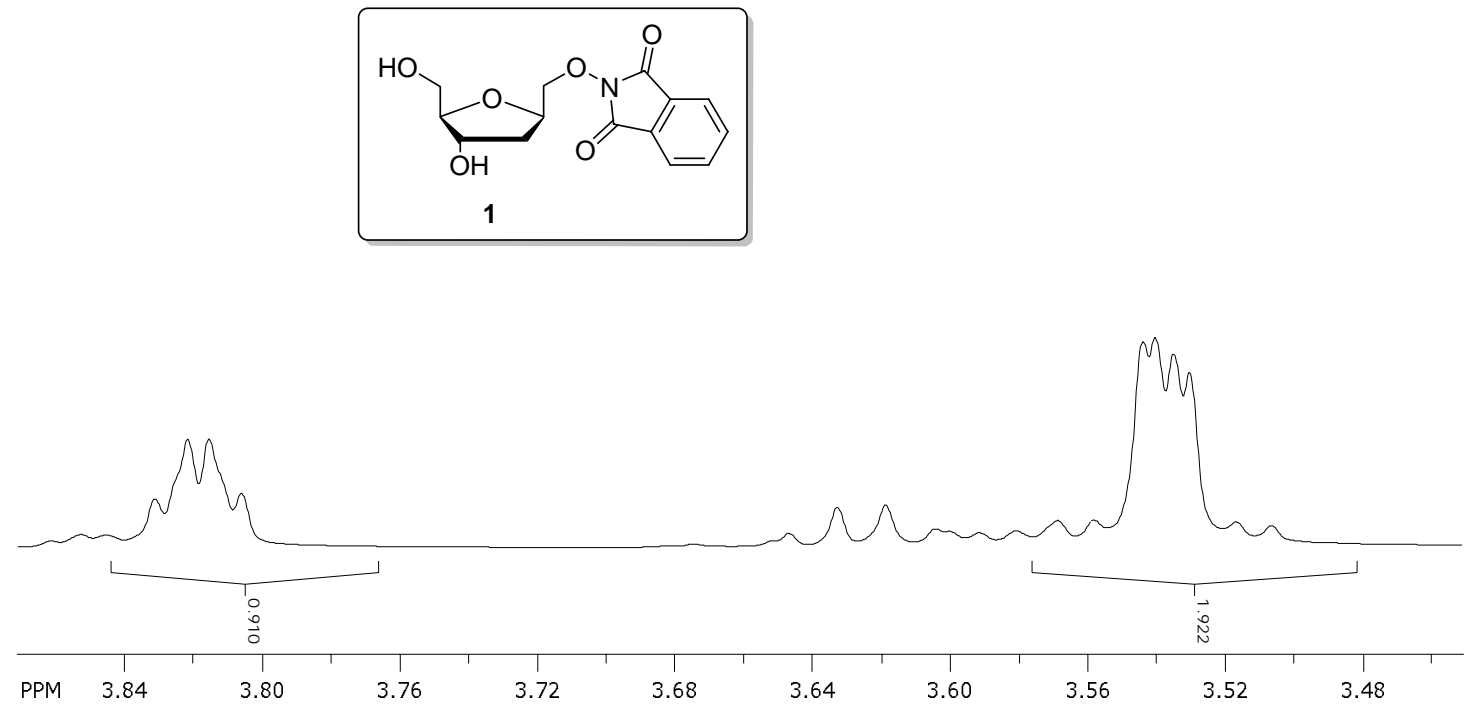

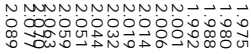

| ||||r|r|rity
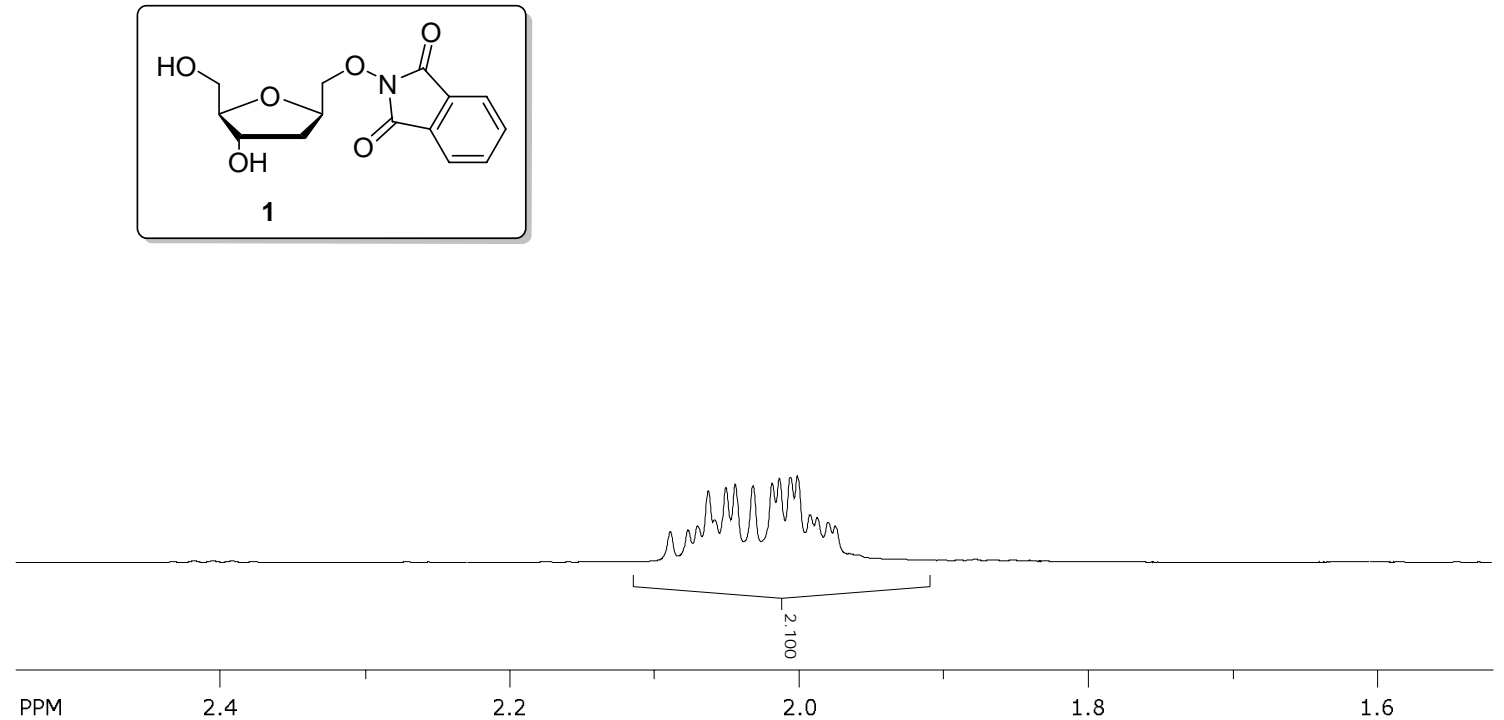

Figure S19. ${ }^{1} \mathrm{H}$ NMR spectrum of compound 1 (continued). 

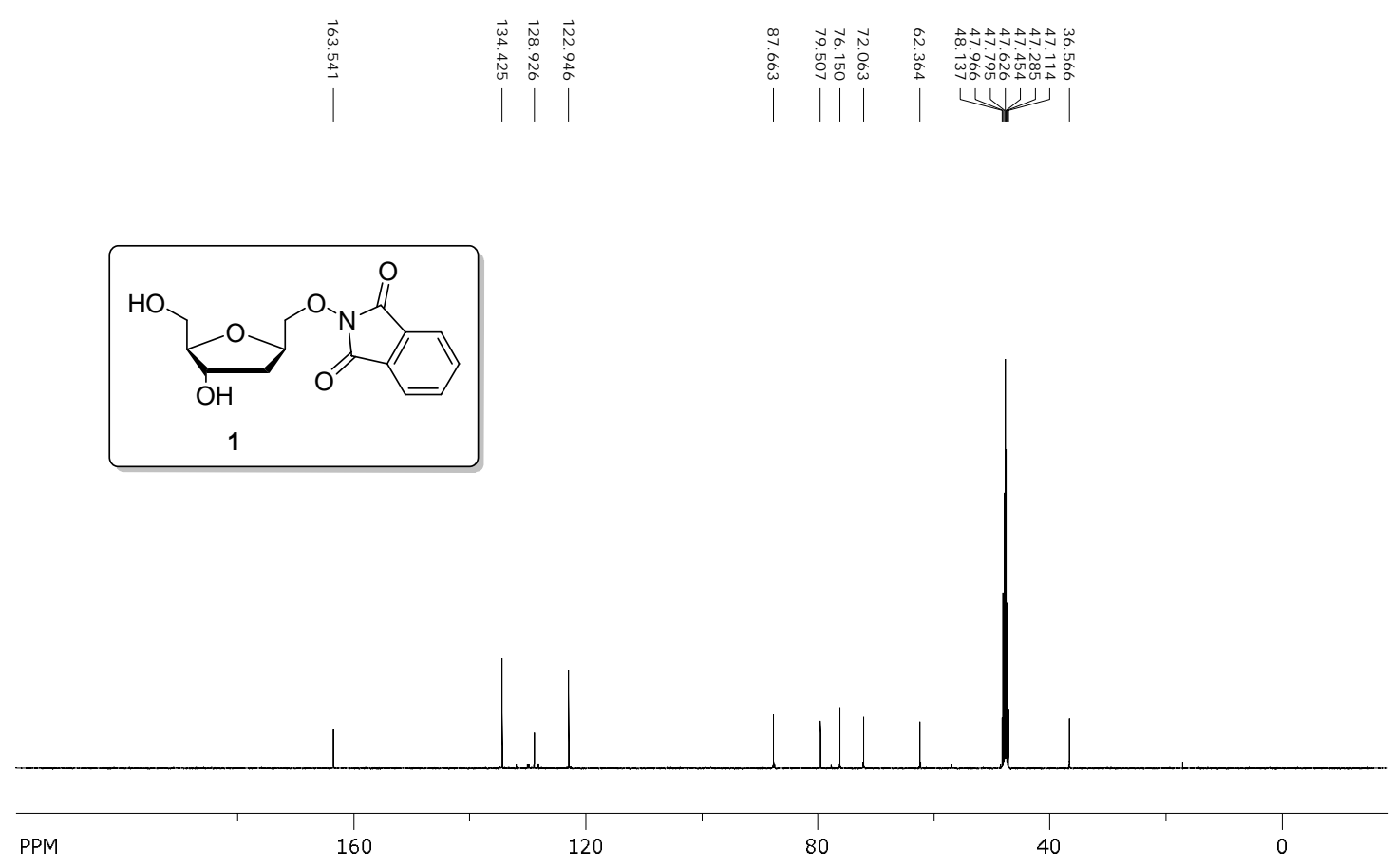

Figure S20. ${ }^{13} \mathrm{C}$ NMR spectrum of compound 1.

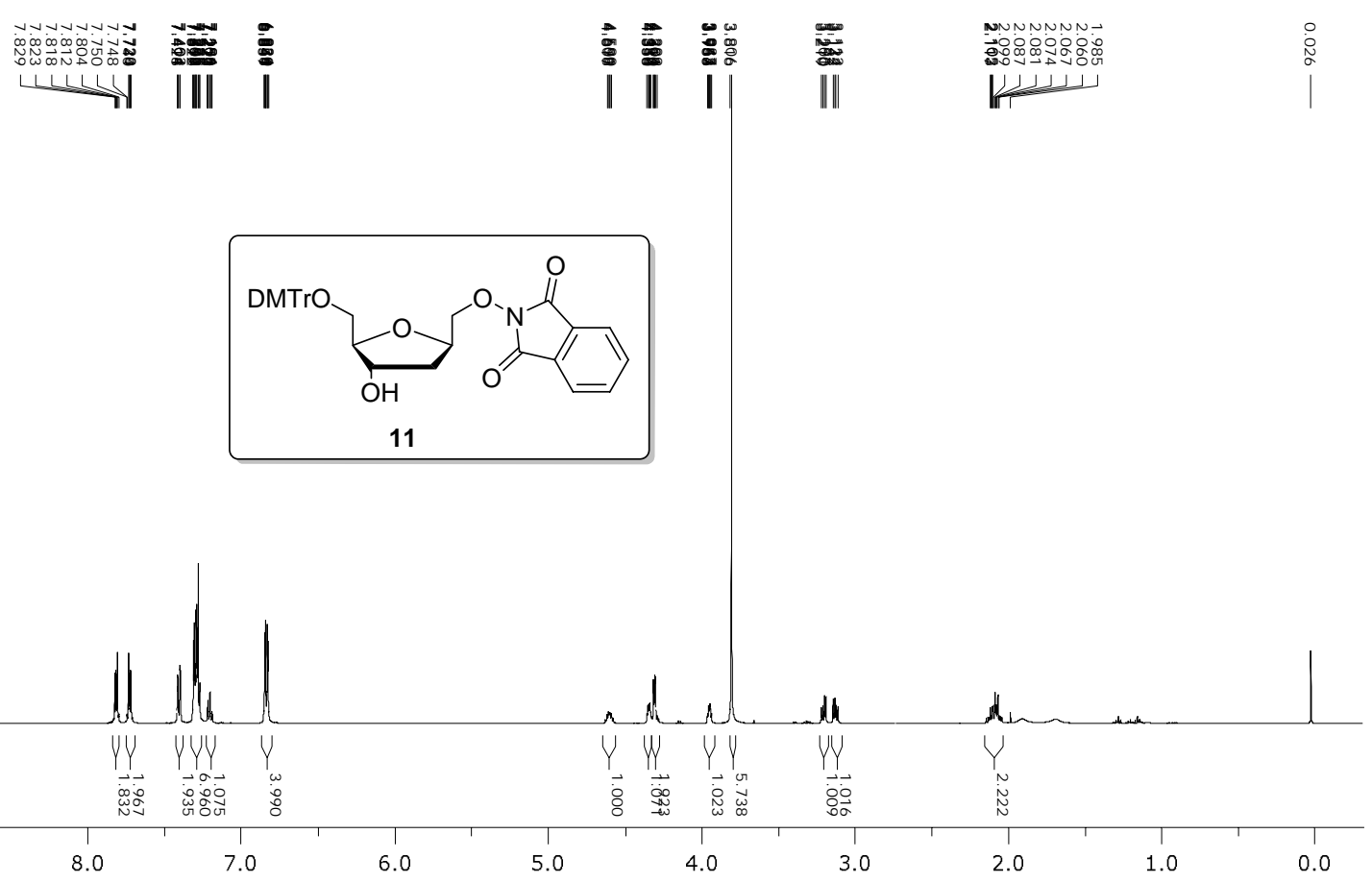

Figure S21. ${ }^{1} \mathrm{H}$ NMR spectrum of compound 11. 

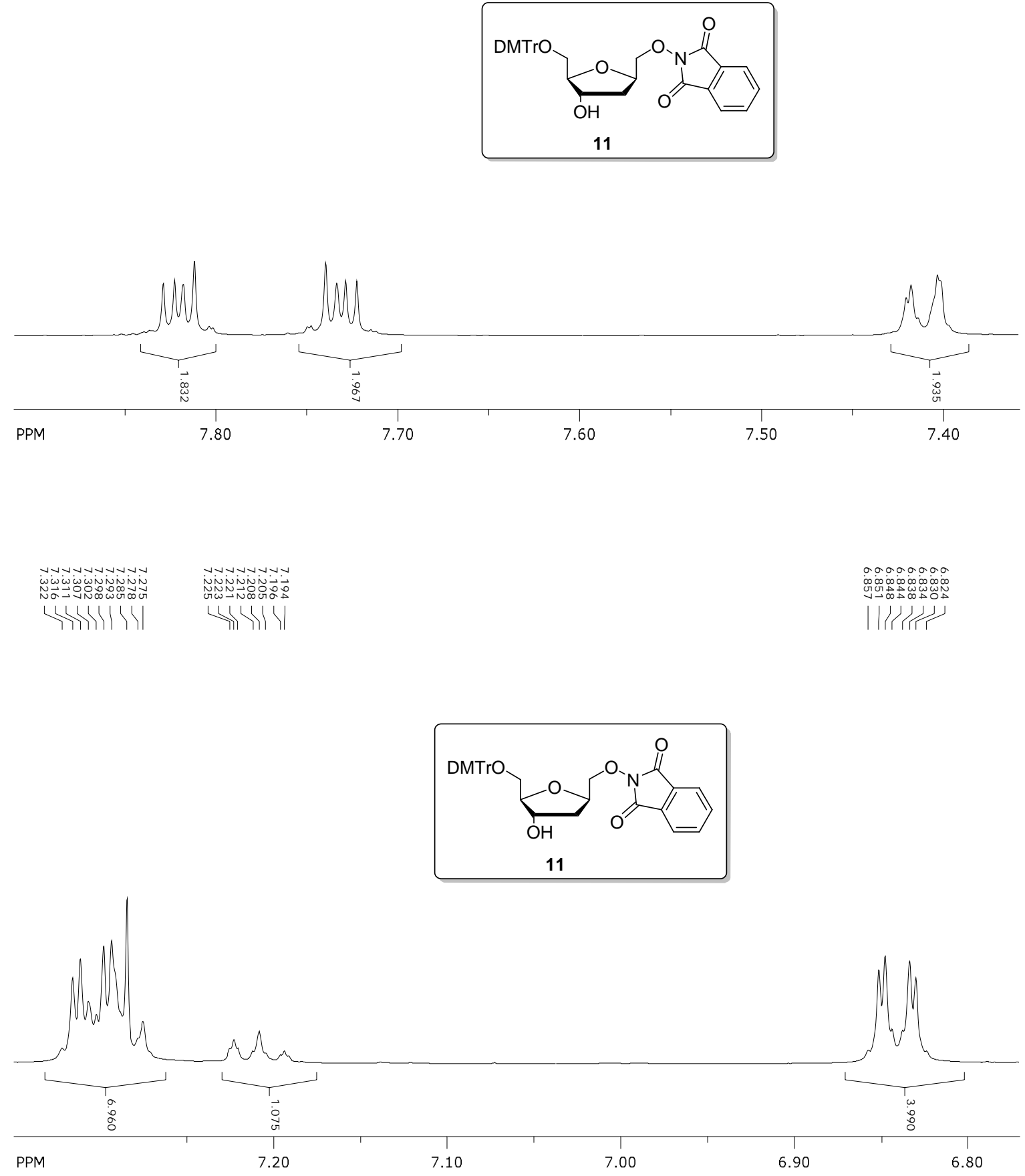

Figure S21. ${ }^{1} \mathrm{H}$ NMR spectrum of compound 11 (continued). 

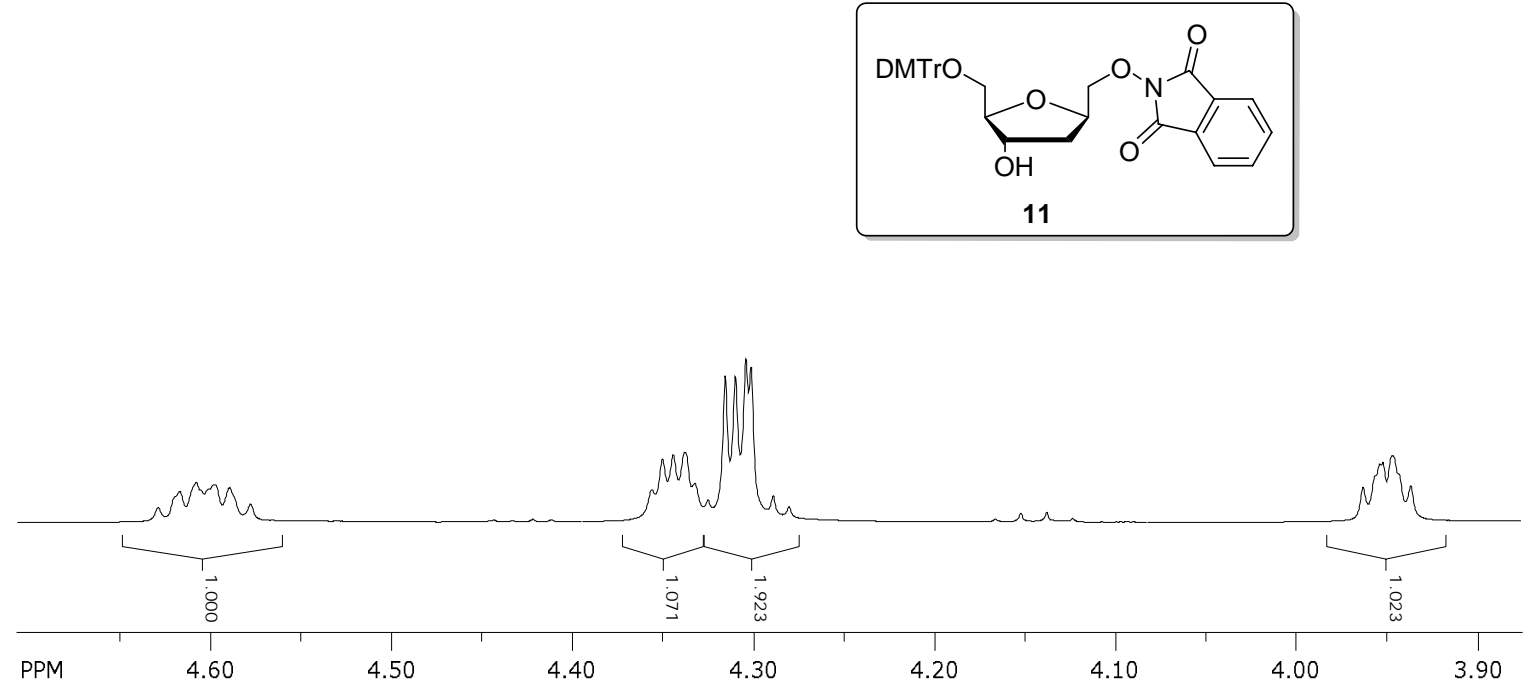

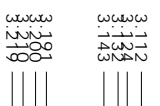

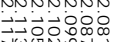
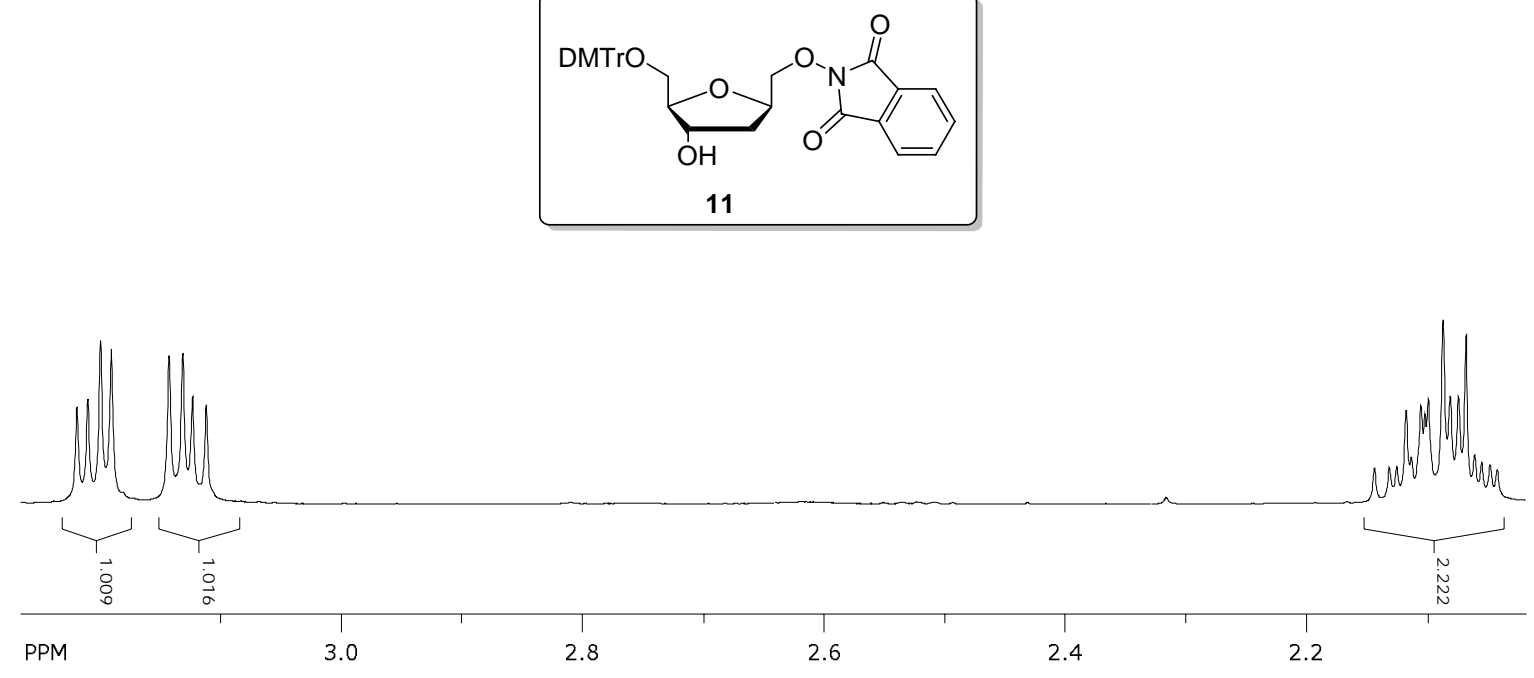

Figure S21. ${ }^{1} \mathrm{H}$ NMR spectrum of compound 11 (continued). 


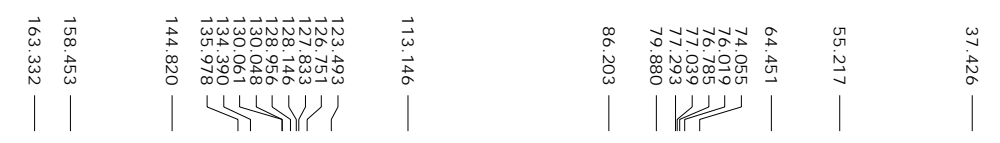
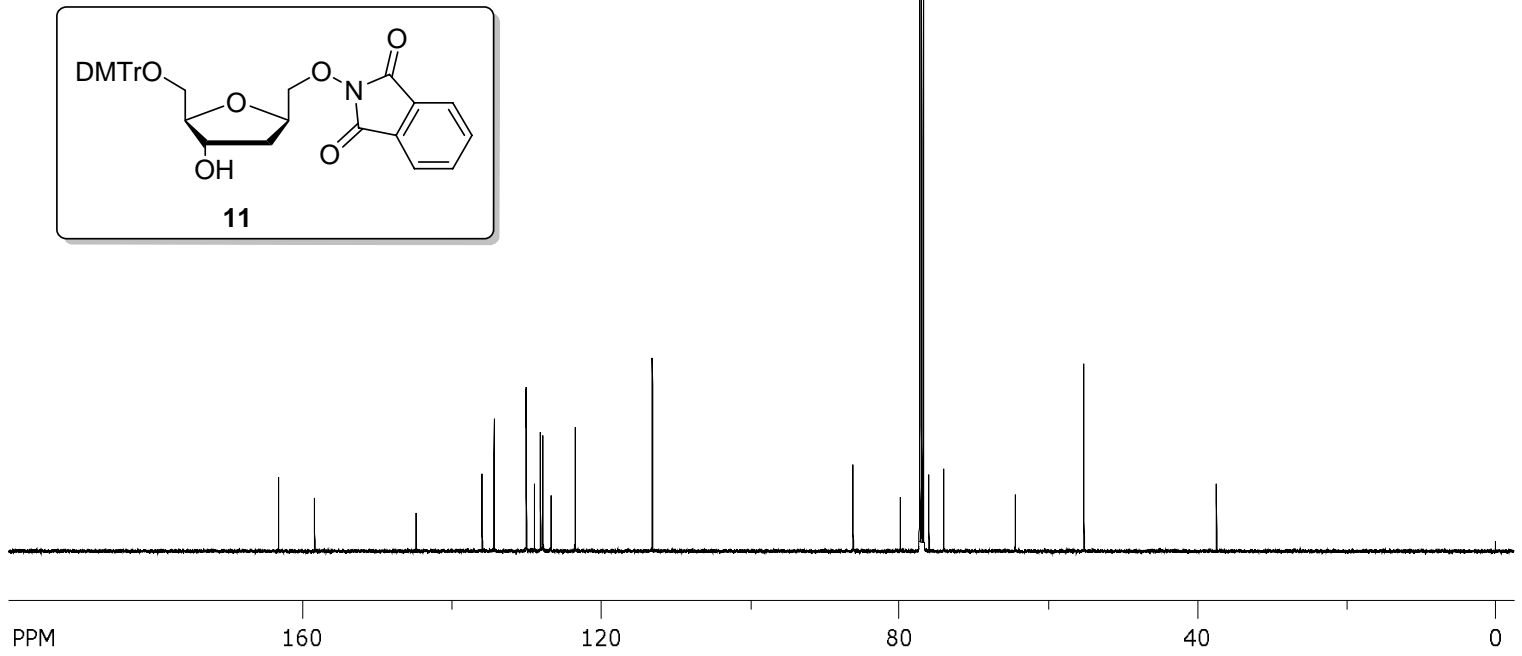

Figure S22. ${ }^{13} \mathrm{C}$ NMR spectrum of compound 11.

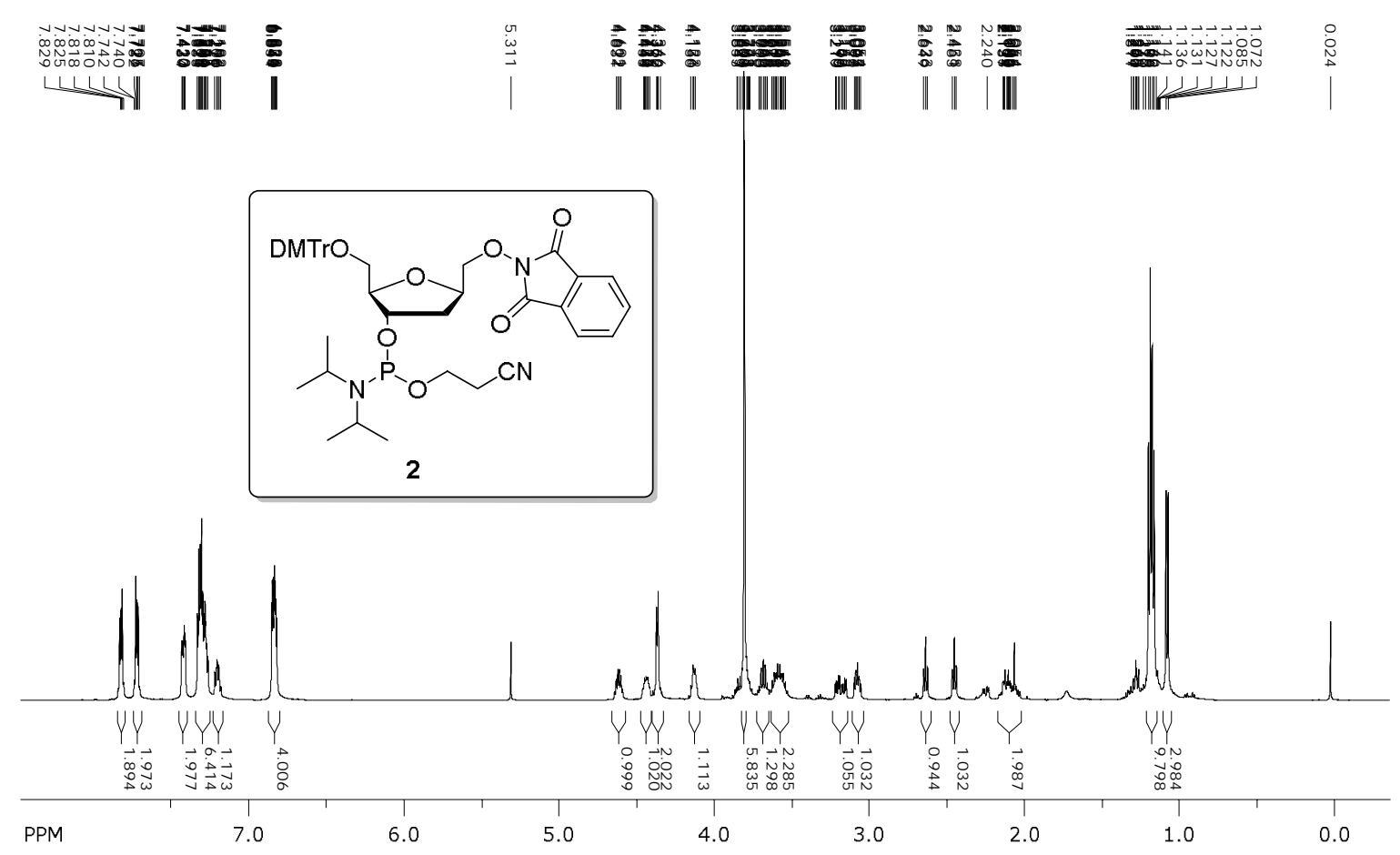

Figure S23. ${ }^{1}$ H NMR spectrum of compound 2. 


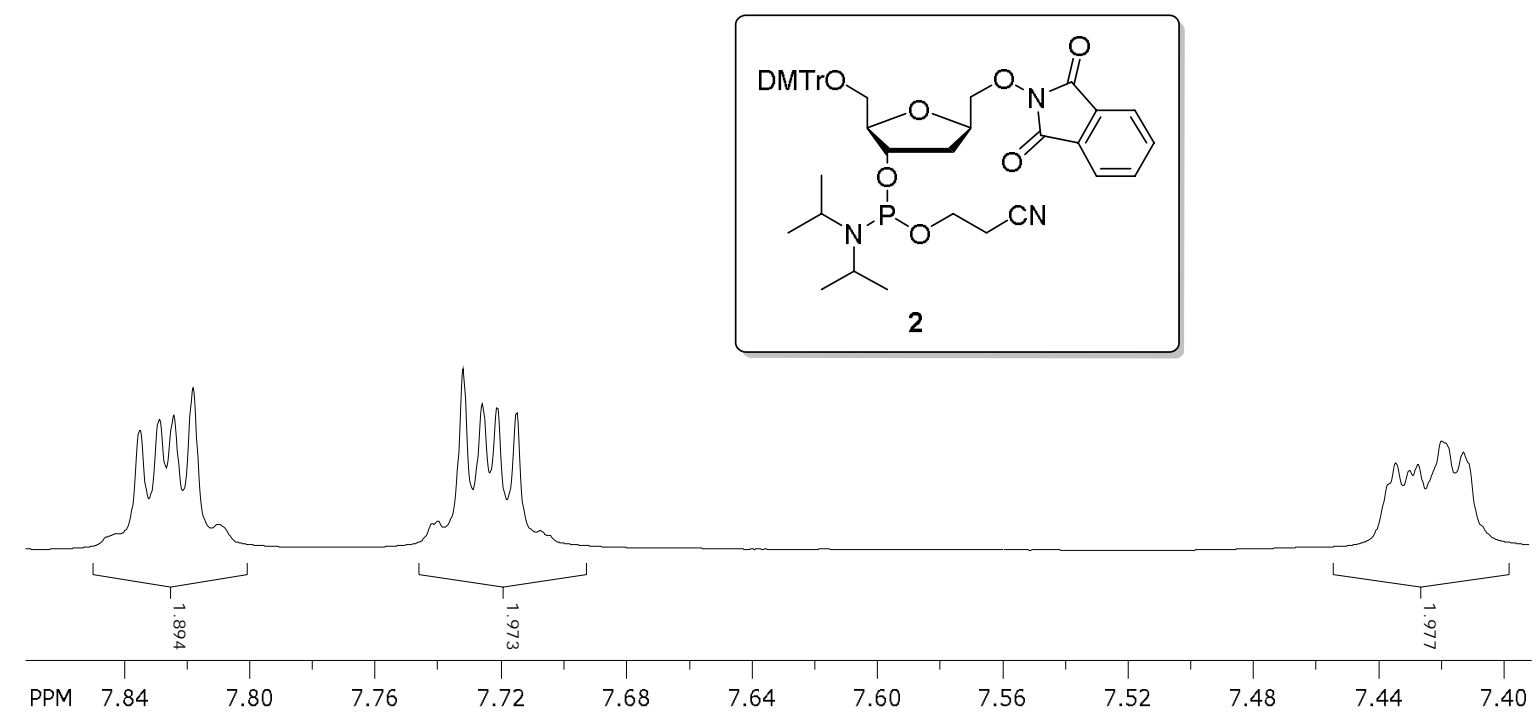

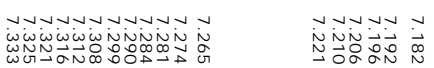

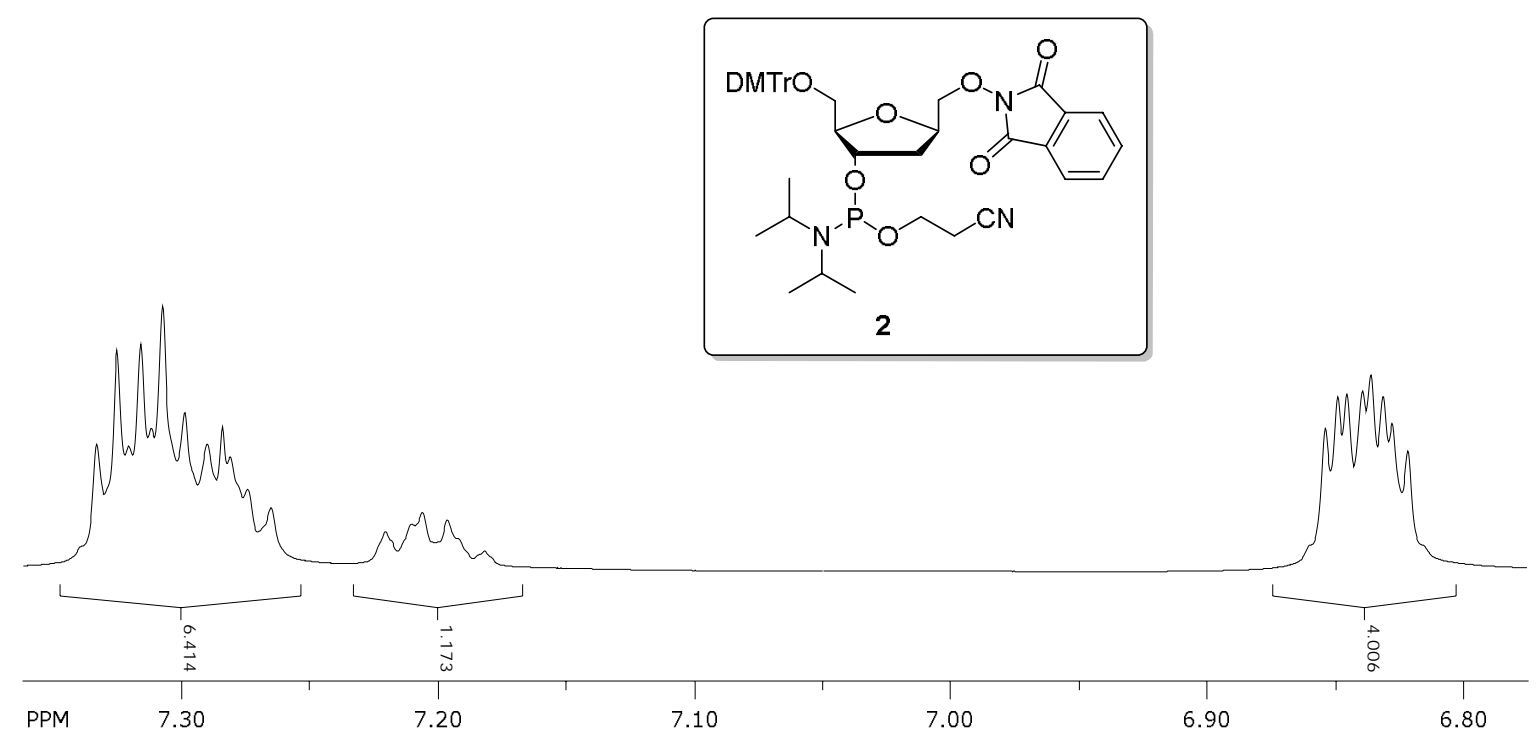

Figure S23. ${ }^{1} \mathrm{H}$ NMR spectrum of compound 2 (continued). 


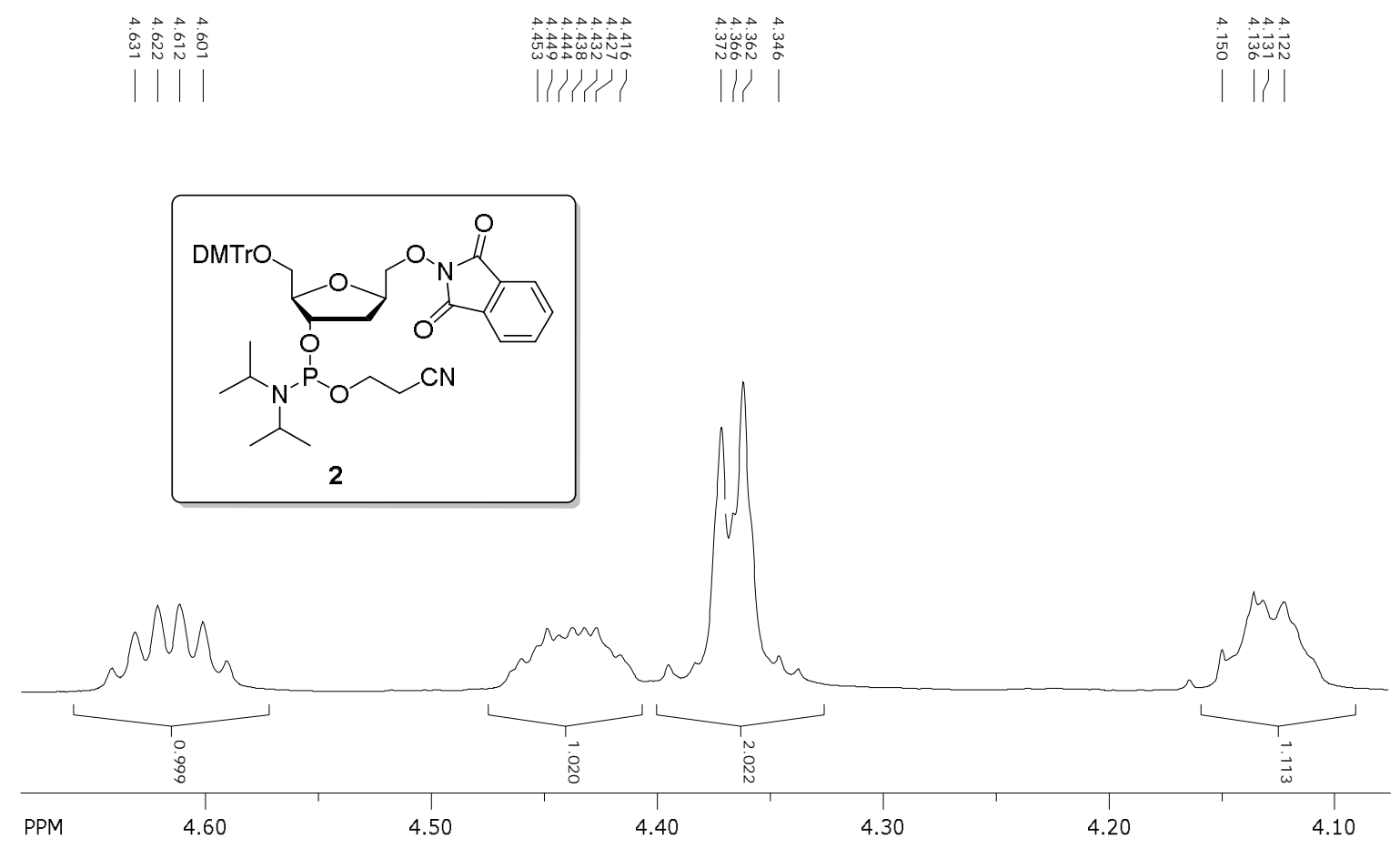

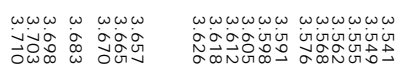

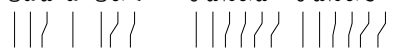

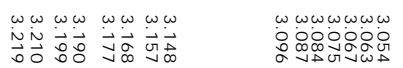

||||||||$\quad \mid 11) \mid 1\}$
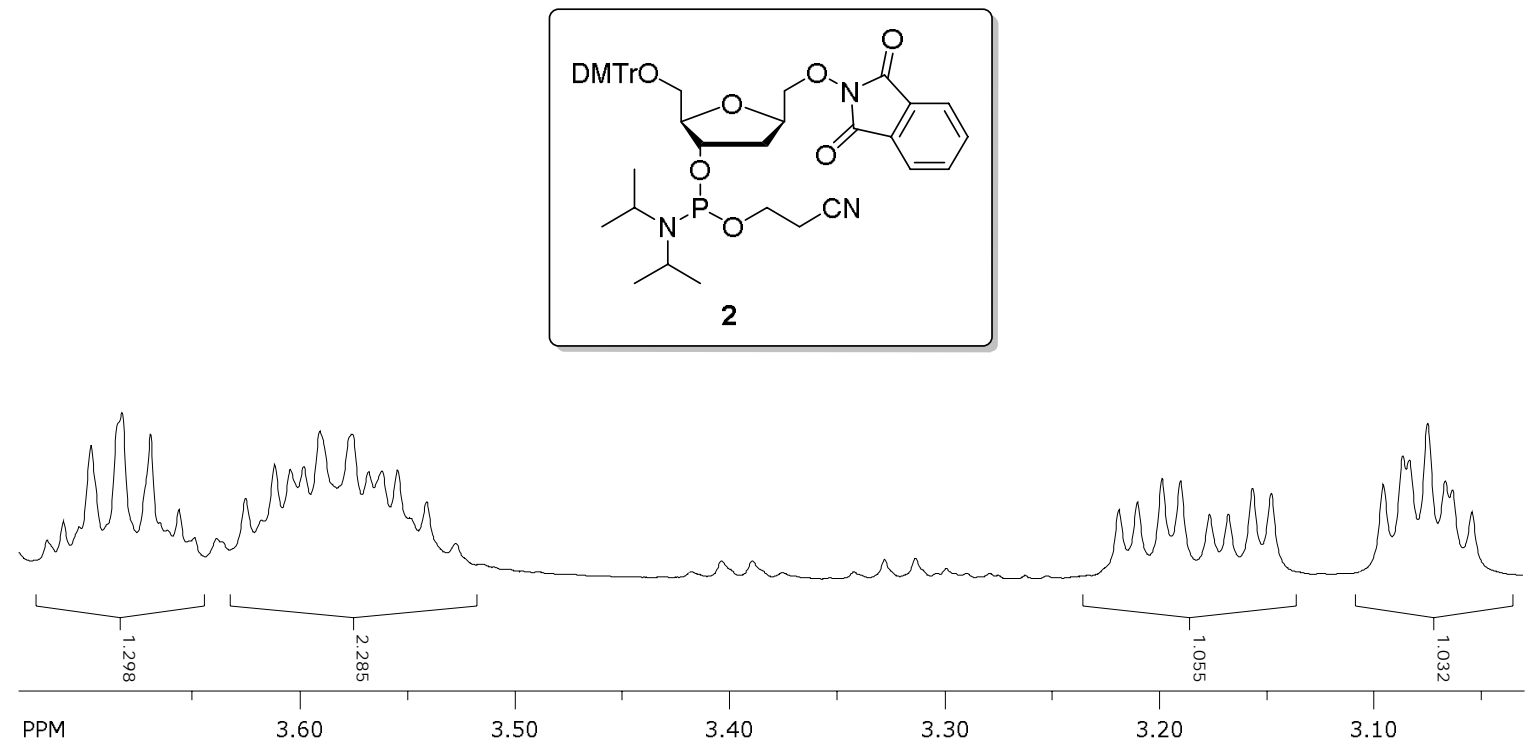

Figure S23. ${ }^{1} \mathrm{H}$ NMR spectrum of compound 2 (continued). 


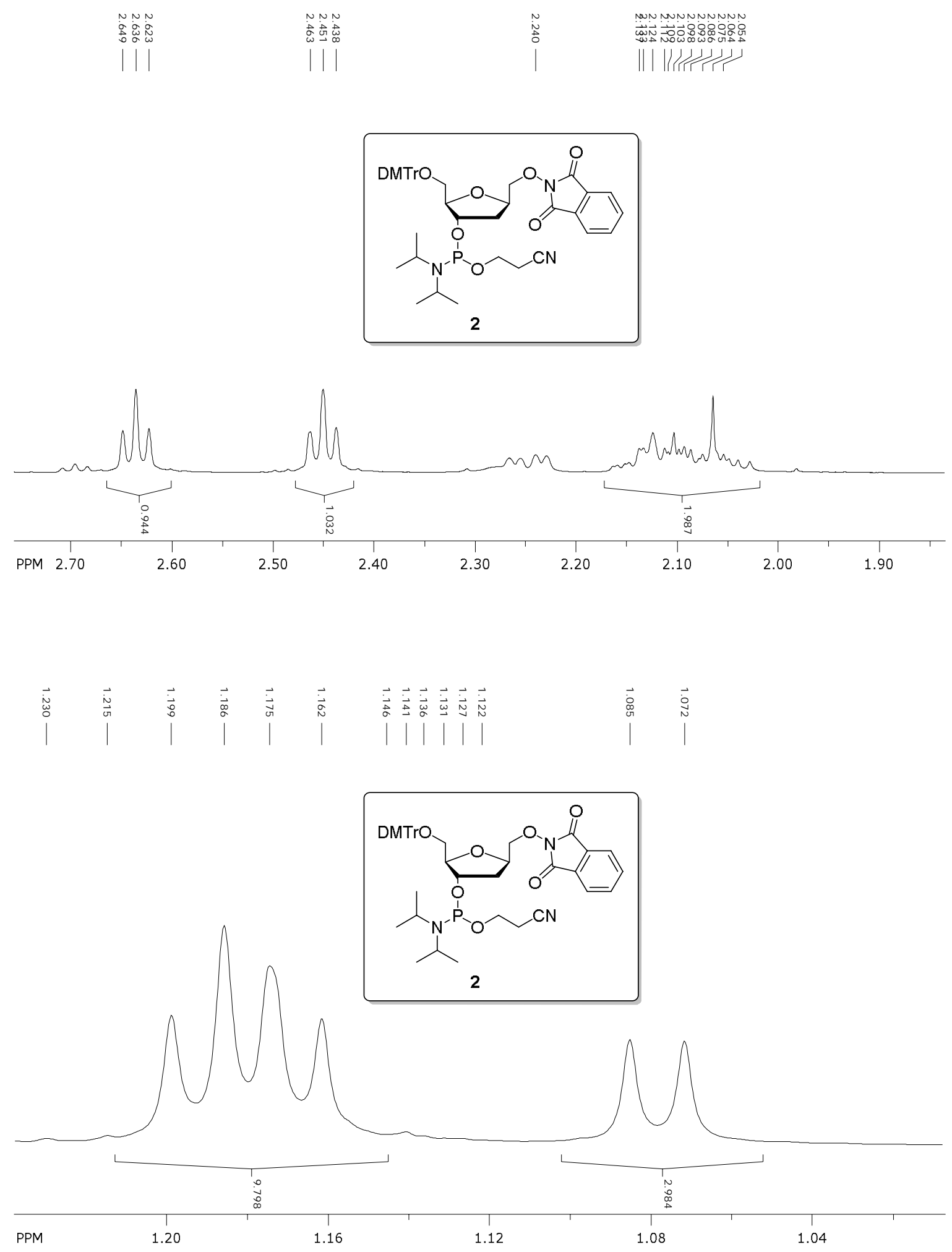

Figure S23. ${ }^{1} \mathrm{H}$ NMR spectrum of compound 2 (continued). 


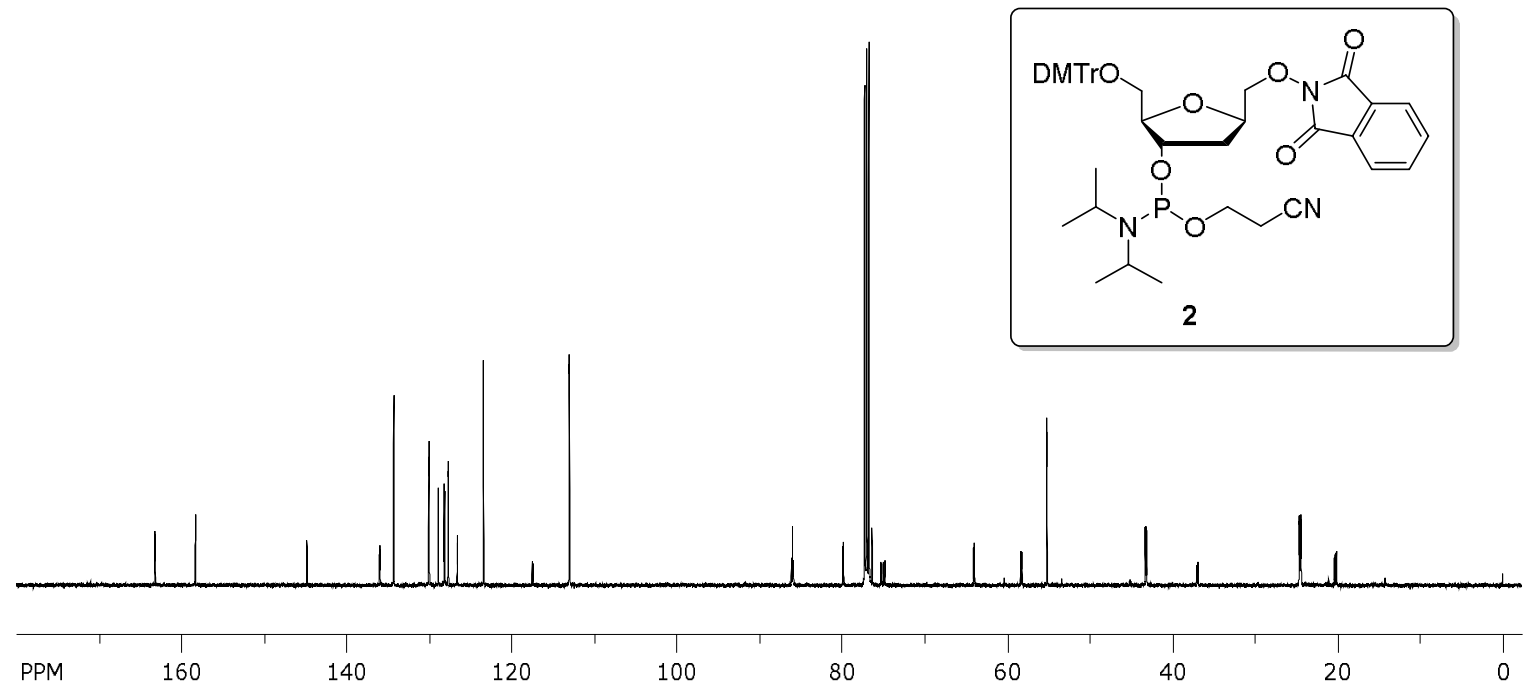

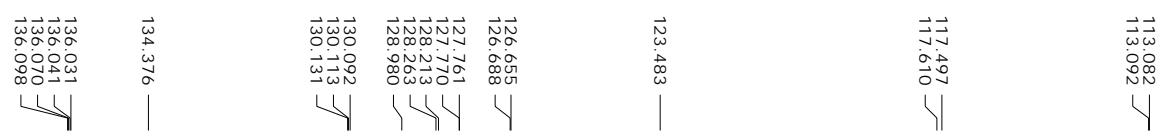
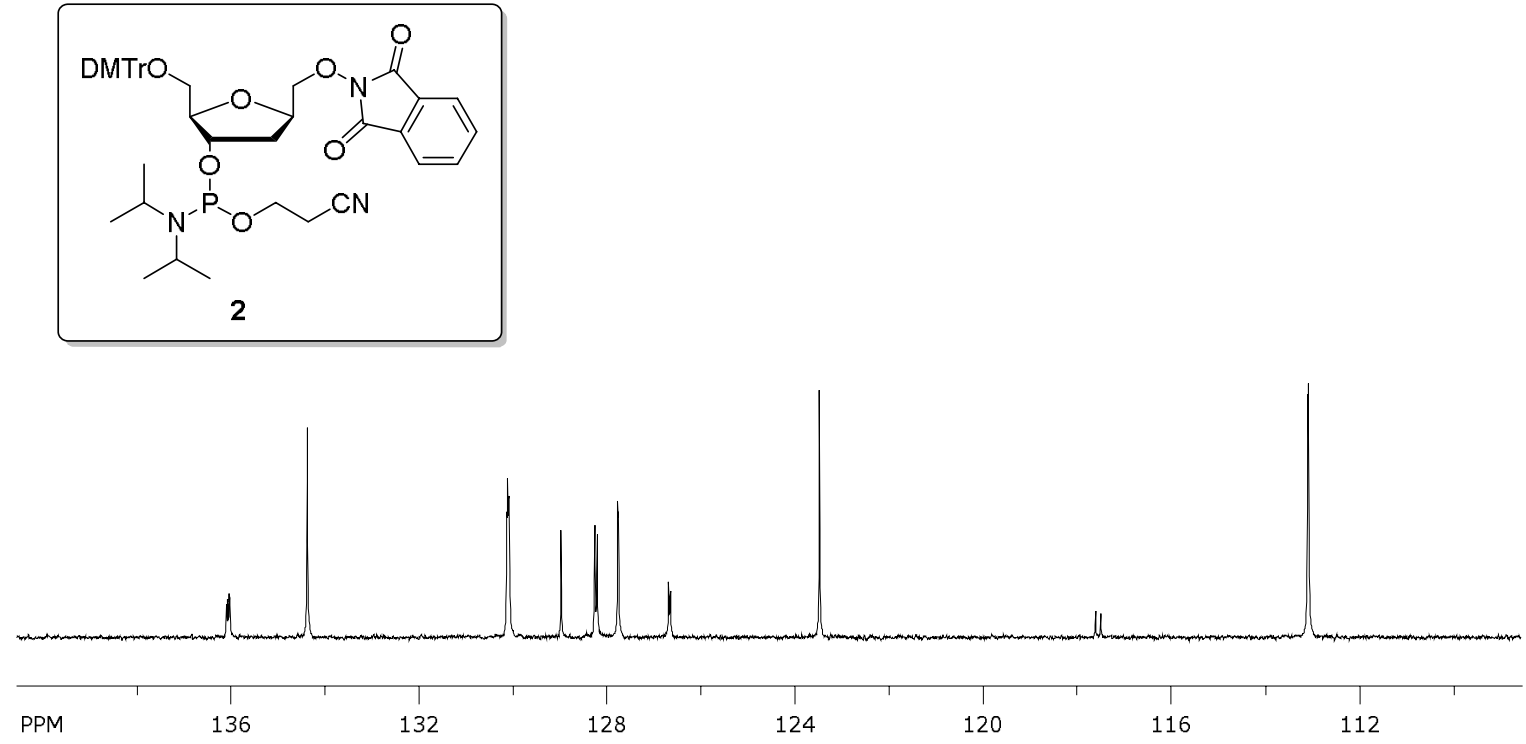

Figure S24. ${ }^{13} \mathrm{C}$ NMR spectrum of compound 2. 


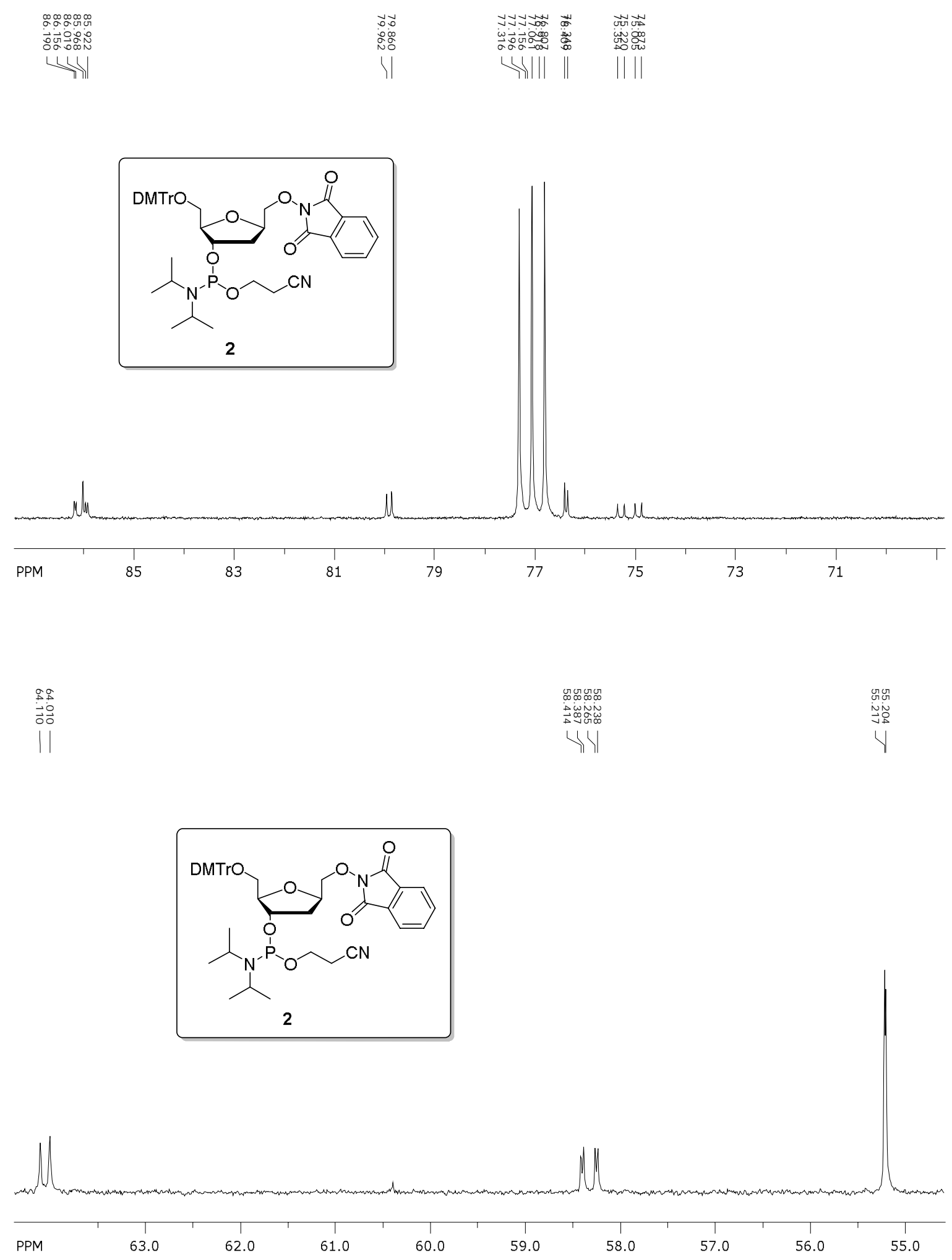

Figure S24. ${ }^{13} \mathrm{C}$ NMR spectrum of compound 2 (continued). 

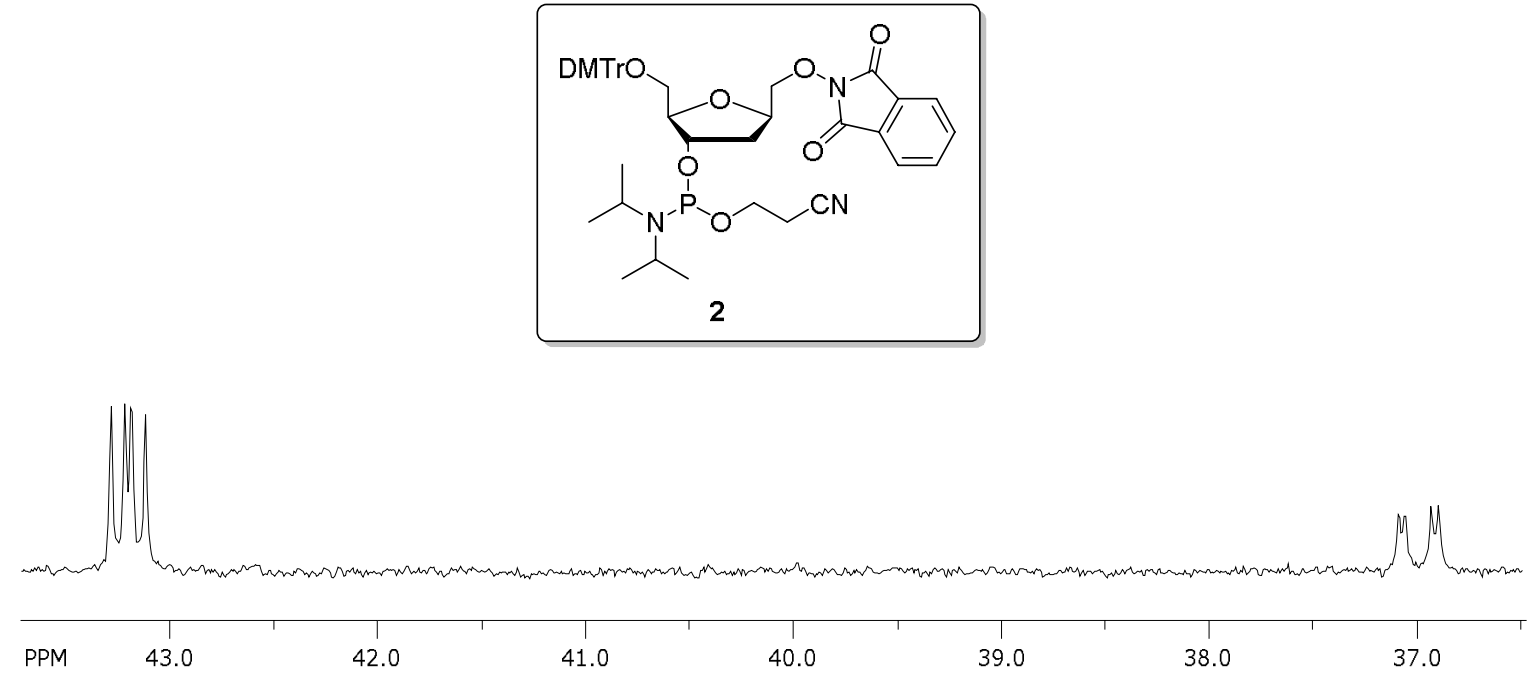

Th+T That

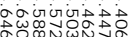

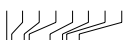

กับ กับ

i⿱⺊口灬心

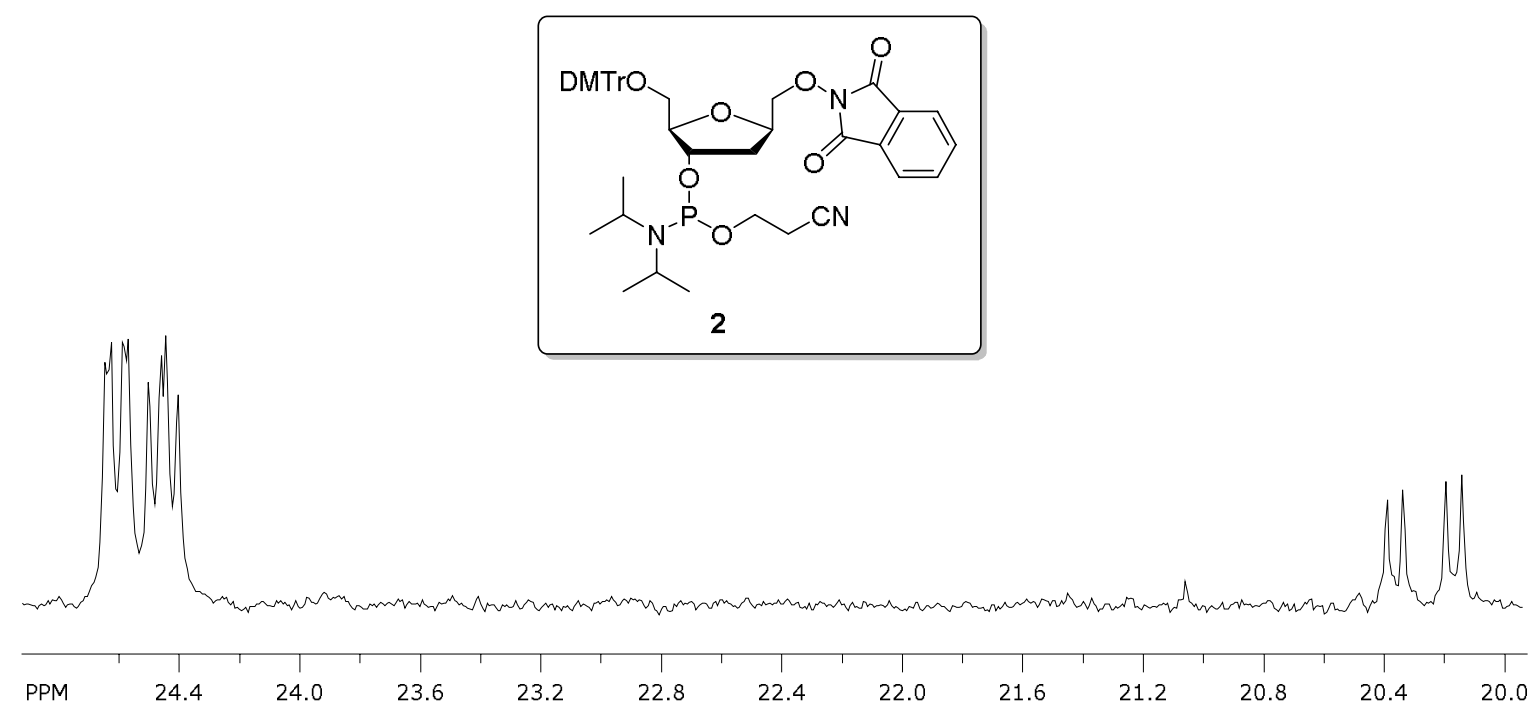

Figure S24. ${ }^{13} \mathrm{C}$ NMR spectrum of compound 2 (continued). 


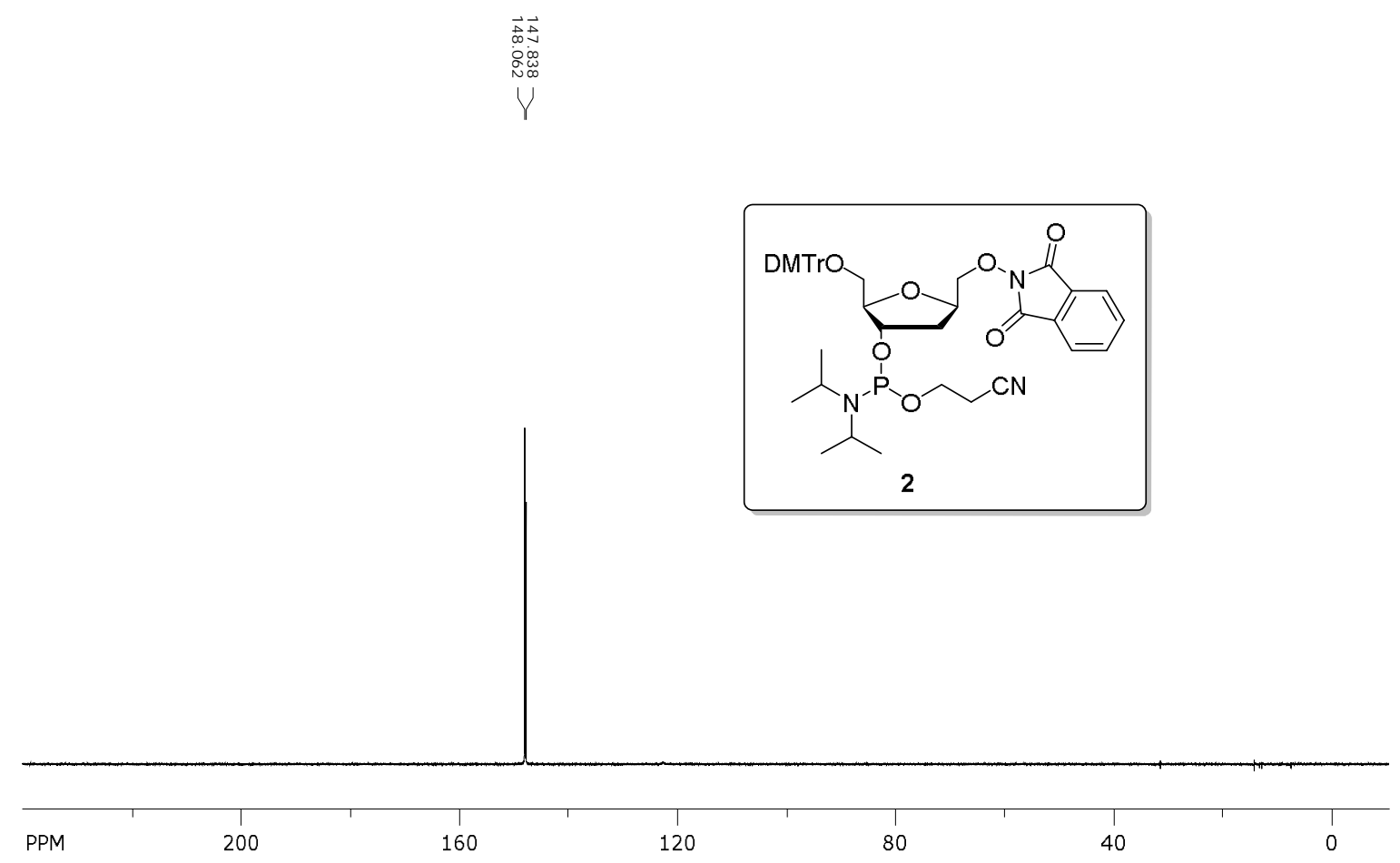

Figure S25. ${ }^{31} \mathrm{P}$ NMR spectrum of compound 2.

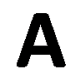

B
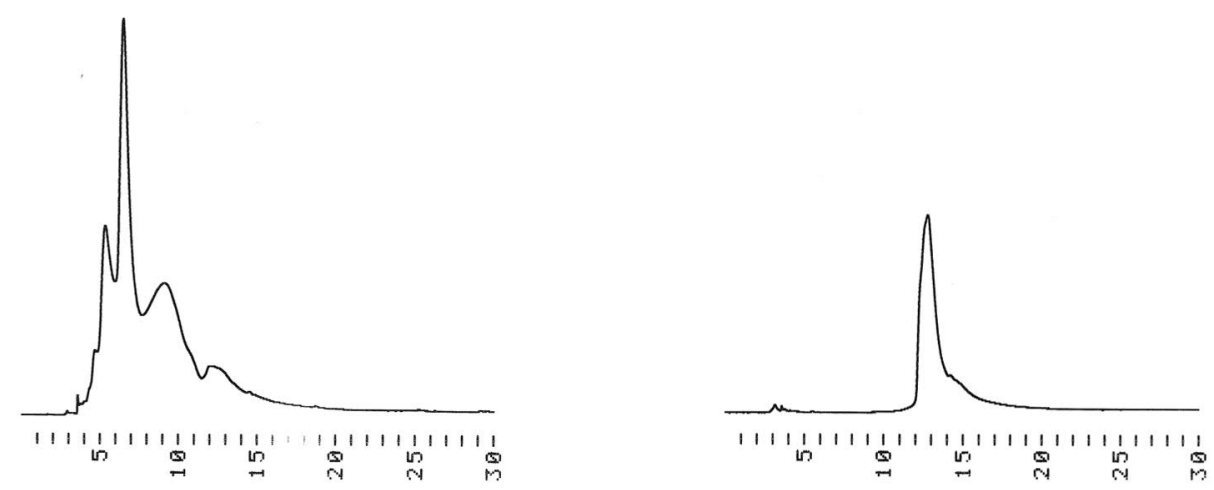

Figure S26. HPLC traces of A) crude and B) purified oligonucleotide ON1-Hg; Hypersil ODS C18 column $(250 \times 4.6 \mathrm{~mm}, 5 \mu \mathrm{m})$; flow rate $=1.0 \mathrm{~mL} \mathrm{~min}^{-1}$; linear gradient $(10$ to $30 \%$ over $30 \mathrm{~min}$ ) of $\mathrm{MeCN}$ in $50 \mathrm{mM}$ aq. triethylammonium acetate. 


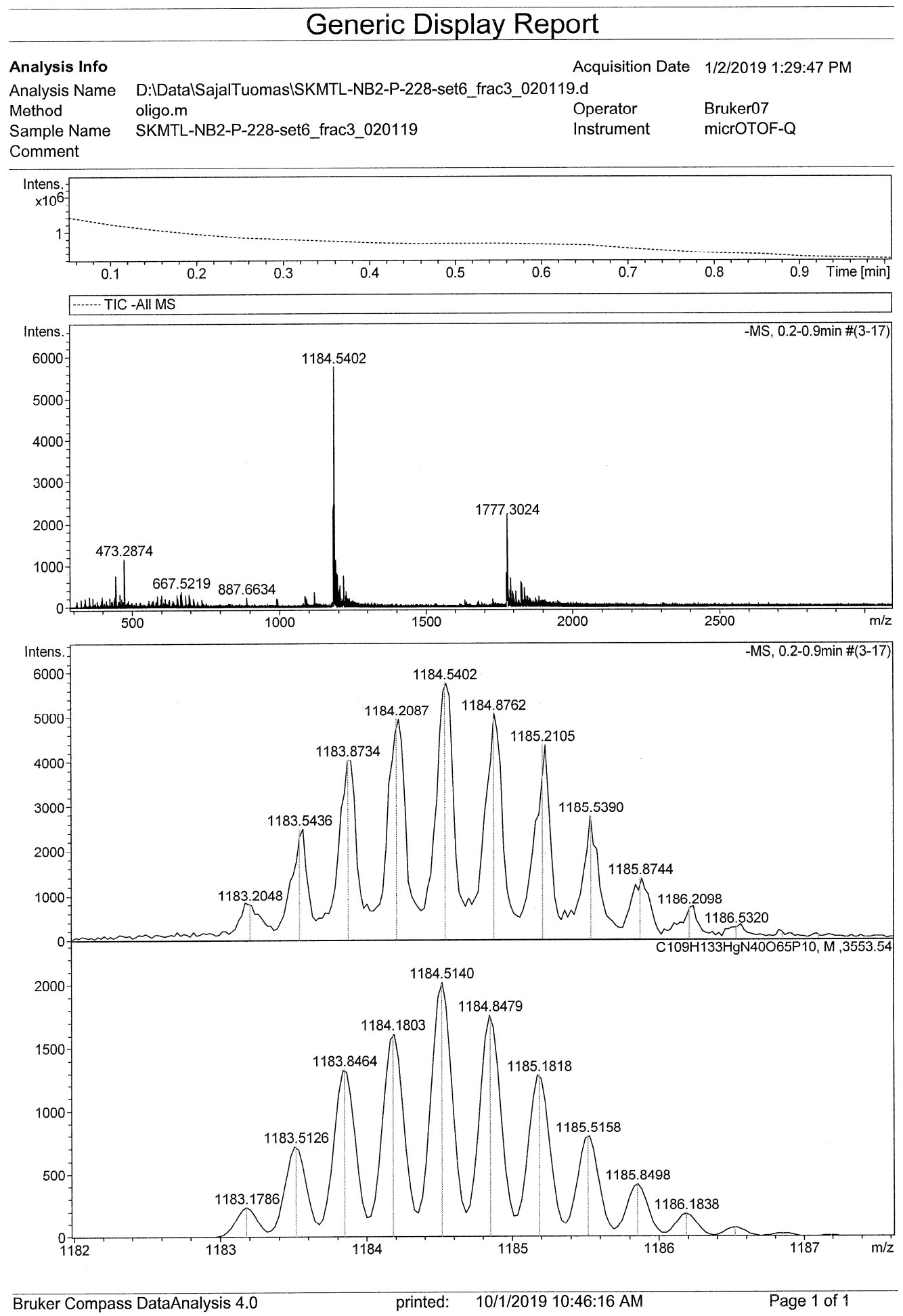

Figure S27. Measured (above) and simulated (below) mass spectra of the purified oligonucleotide ON1-Hg; $m / z$ : calcd for $\mathrm{C}_{109} \mathrm{H}_{133} \mathrm{HgN}_{40} \mathrm{O}_{65} \mathrm{P}_{10}[\mathrm{M}-4 \mathrm{H}]^{3-}:$ 1184.5140; found: 1184.5402. 


\section{A}

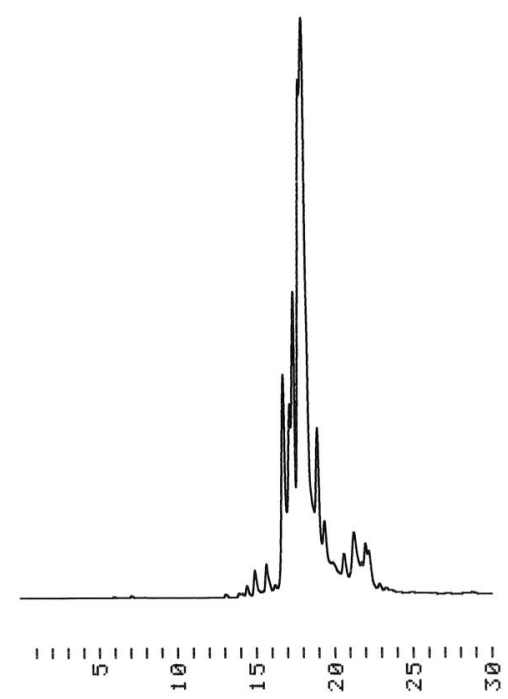

B

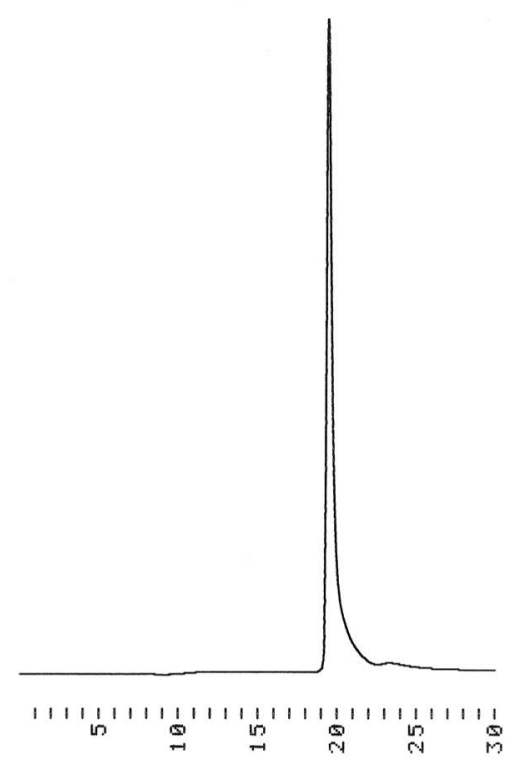

Figure S28. HPLC traces of A) crude and B) purified oligonucleotide ON1-Pd; Hypersil ODS C18 column $(250 \times 4.6 \mathrm{~mm}, 5 \mu \mathrm{m})$; flow rate $=1.0 \mathrm{~mL} \mathrm{~min}^{-1}$; linear gradient ( 5 to $30 \%$ over $30 \mathrm{~min}$ ) of $\mathrm{MeCN}$ in $50 \mathrm{mM}$ aq. triethylammonium acetate.

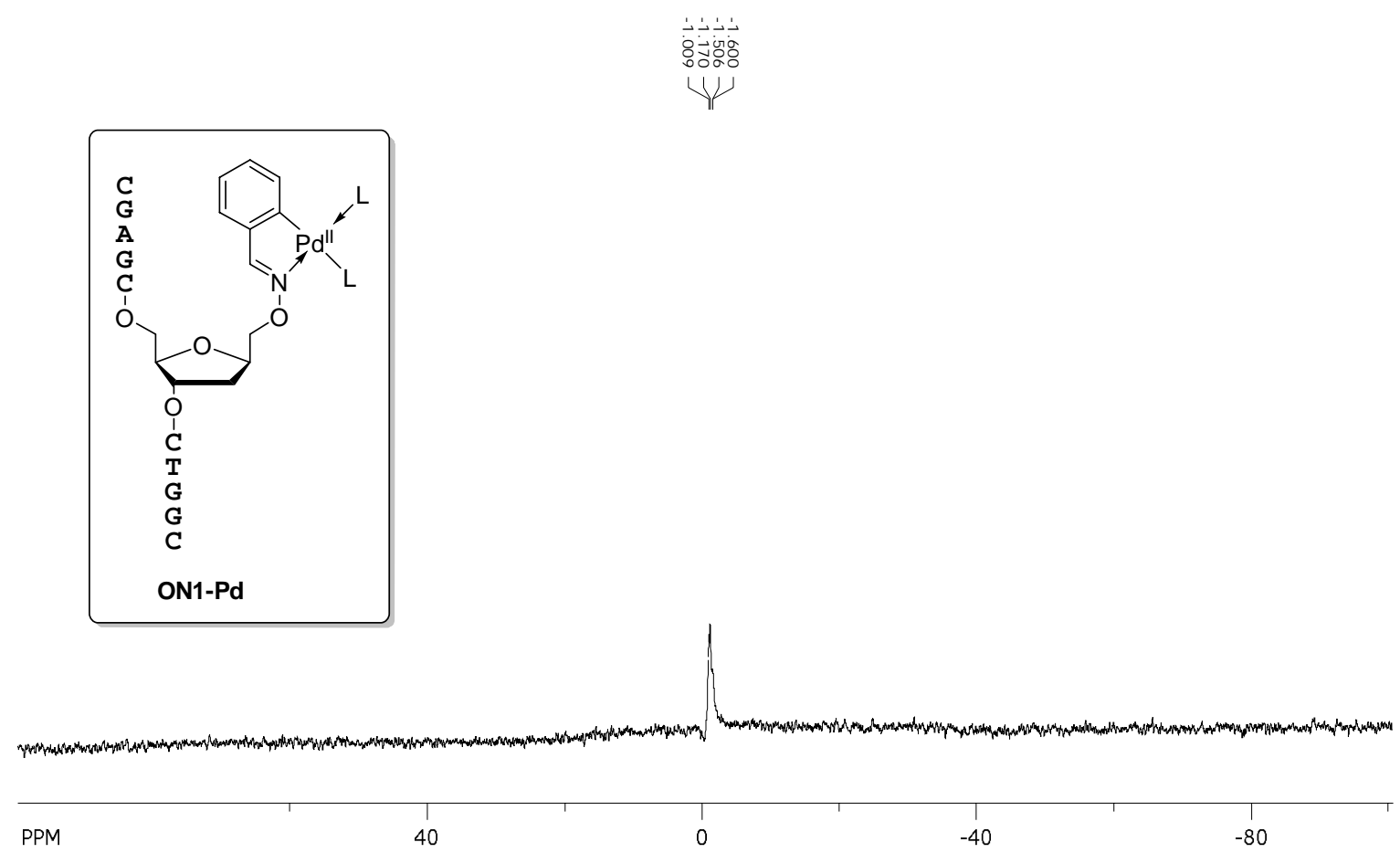

Figure S29. ${ }^{31} \mathrm{P}$ NMR spectrum of purified oligonucleotide ON1-Pd. 


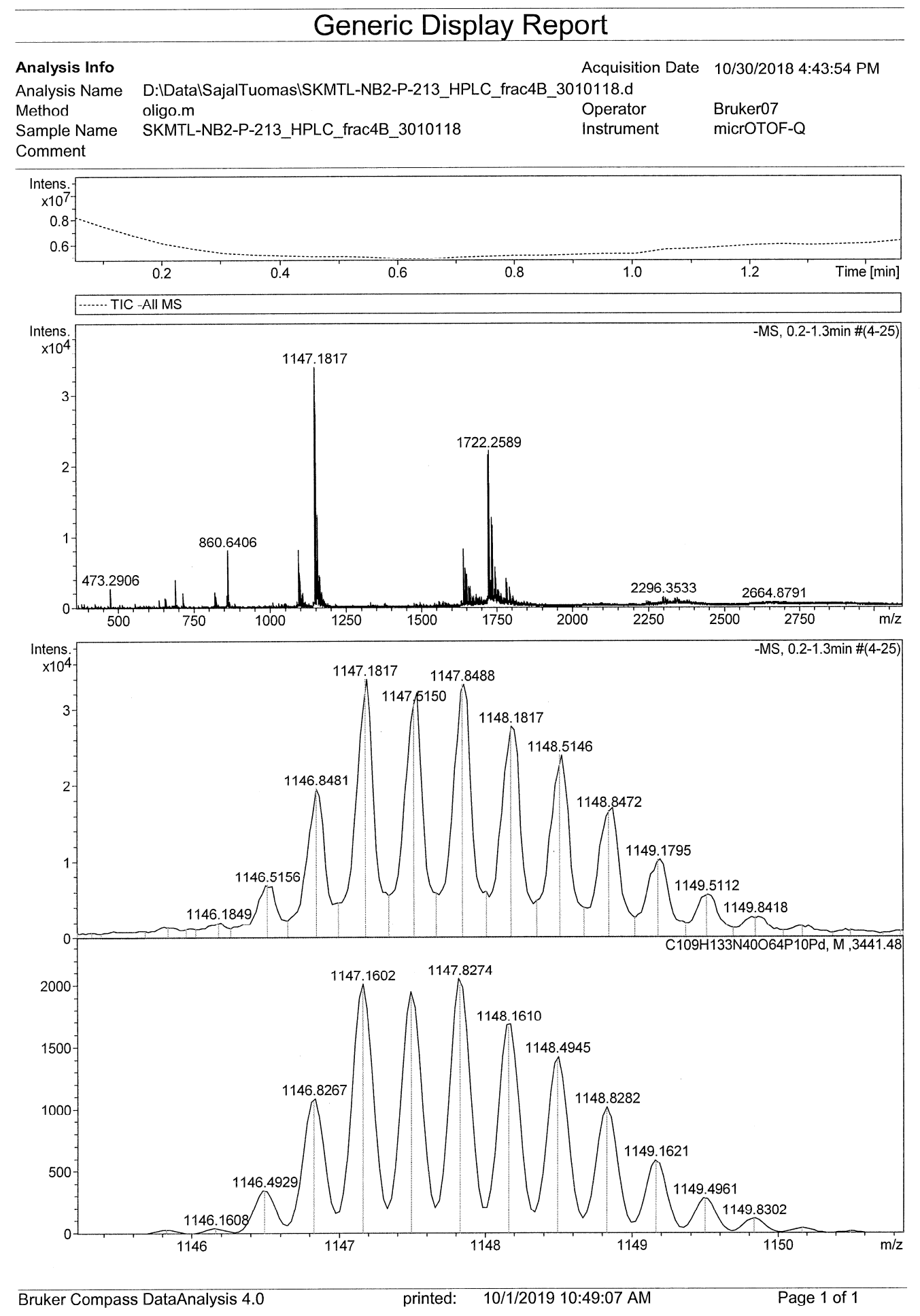

Figure S30. Measured (above) and simulated (below) mass spectra of the purified oligonucleotide ON1-Pd; $m / z$ : calcd for $\mathrm{C}_{109} \mathrm{H}_{133} \mathrm{~N}_{40} \mathrm{O}_{64} \mathrm{P}_{10} \mathrm{Pd}[\mathrm{M}-4 \mathrm{H}]^{3-}:$ 1147.1602; found: 1147.1817. 